\title{
Locomotion grows up: The neuromechanical control of interlimb coordinating mechanisms in crayfish
}

Marc D. Rinehart

West Virginia University

Follow this and additional works at: https://researchrepository.wvu.edu/etd

\section{Recommended Citation}

Rinehart, Marc D., "Locomotion grows up: The neuromechanical control of interlimb coordinating mechanisms in crayfish" (2009). Graduate Theses, Dissertations, and Problem Reports. 2942.

https://researchrepository.wvu.edu/etd/2942

This Dissertation is protected by copyright and/or related rights. It has been brought to you by the The Research Repository @ WVU with permission from the rights-holder(s). You are free to use this Dissertation in any way that is permitted by the copyright and related rights legislation that applies to your use. For other uses you must obtain permission from the rights-holder(s) directly, unless additional rights are indicated by a Creative Commons license in the record and/ or on the work itself. This Dissertation has been accepted for inclusion in WVU Graduate Theses, Dissertations, and Problem Reports collection by an authorized administrator of The Research Repository @ WVU.

For more information, please contact researchrepository@mail.wvu.edu. 


\title{
Locomotion Grows Up:
}

The Neuromechanical Control of Interlimb Coordinating Mechanisms in Crayfish

Marc D. Rinehart

\author{
Dissertation submitted to the Eberly College of Arts and Sciences \\ at West Virginia University \\ in partial fulfillment of the requirements \\ for the degree of

\section{Doctor of Philosophy} \\ in \\ Biology
}

Jim H. Belanger, Ph.D., Chair

John Caprio, Ph.D.

Kevin Daly, Ph.D.

Sarah Farris, Ph.D.

Michelle Withers, Ph.D.

Department of Biology

Morgantown, West Virginia

2009

Keywords: Behavior; Walking; Arthropods; Crustacea

Copyright 2009 Marc D. Rinehart 


\section{Abstract \\ Locomotion Grows Up: The Neuromechanical Control of Interlimb Coordinating Mechanisms in Crayfish}

\section{Marc D. Rinehart}

Locomotion requires many dynamic interactions between organism and environment at several levels. It is not known how the nervous system controls all of these relationships to ultimately produce and guide locomotor behavior. Furthermore, it is not known whether the nervous system needs to recognize and control all of the possible body-environment interactions. In this study the crayfish (Procambarus clarkii) is used as a model system to test how size influences locomotor behavior and how a single, simplified neuromechanical system can accommodate these changes.

A set of behavioral experiments was conducted to characterize kinematics of freely walking juvenile crayfish to compare with adults. The purpose of these studies was to determine how crayfish adapt to a great change in size during their ontogeny. Juvenile and adult crayfish show differences in limb function and coordination. Although crayfish are decapods, the juveniles predominantly use the posterior legs and behave more like four-legged walkers. The difference in locomotor behavior can best be explained by differences in chelae size. Allometric relationships between juveniles and adults show limb and body morphologies scale proportionately. Adult chelae, or claws, are twice as long and contribute $\sim 20 \%$ more to the total body mass in fully mature crayfish. This increase in chelae size shifts the location of the center of mass anterior as crayfish grow. The result is a change in relative load distribution that appears to affect individual limb behavior and interlimb coordination. Shifting the center of mass in adults by amputating the chelae resulted in limb behavior and interlimb coordination more similar to that observed in juveniles. Likewise, applying load to the rostrum of juveniles altered behavior and changed limb function in the posterior legs similar to adults with large chelae. The results of these experiments suggest that crayfish of all sizes adapt to changes in load distribution by adjusting behavior of individual legs.

To test whether developmental influences have an effect on walking behavior, juveniles were induced to walk on a treadmill at various speeds. The animals showed more consistent limb coordination as walking speed increased, similar to adults. Selected legs were then amputated to test how gait was affected. Amputating legs removes sensory feedback from the distal leg to the central nervous system. The behavior of the stump is therefore more representative of the endogenous rhythmicity of the central pattern generator (CPG). Juveniles showed no differences in coordination in individual legs. Coordination between adjacent ipsilateral legs was also the same as that observed in adults following amputation. Furthermore, intact legs acquired new interlimb coordination similar to adults. These results suggested that juvenile and adult crayfish have functionally similar nervous systems controlling walking.

Finally, a 3-D virtual crayfish was built to test whether differences in walking between juveniles and adults could be due to mechanical influences alone. The model crayfish lacked direct connections between legs. The model responded to shifts in the center of mass by showing more consistent limb coordination in those legs nearest the center of mass. This was achieved through 
indirect mechanical coupling of the legs through the environment and body of the crayfish. This mechanism also produced realistic adaptive behavior when limbs were amputated. This showed that differences between adult and juvenile walking are due solely to mechanical influences associated with the changing center of mass as the animals grow. These results suggest further that organisms do not need high levels of control to produce coordinated behavior. Locomotor behavior arises through interactions between body, limb, and environment that are a function of the spatio-temporal dynamics of body morphology. The results may be applicable to a large number of walking systems. 
To organ donors and the lives they have touched 


\section{Acknowledgments}

I would like to first acknowledge my fellow scienticians, Richard and Andrés. I could not have asked for better lab mates. You provided guidance and motivation and you showed me the necessity of emergency lab meetings at Chelsea's. I also have to thank my fellow graduate students at LSU, Shane, Emily, Scott, and Merve. Your insights and venting sessions were invaluable. To all of the graduate students at WVU, thank you for taking me in as one of your own and making the most stressful part of this process bearable.

I have to thank everyone who supported me at LSU. I wish that I could have finished this with you but know that I could not have made it the last few years without you. Kurt, your work ethic and enthusiasm are contagious. Evanna, your dedication to your students is inspirational. Jan, your integrity will not be forgotten. To my newest committee members, thank you for accommodating my unique situation. Kevin, thank you for guidance and perspective (and numerous signatures). Sarah, your love for science has provided daily motivation and a hope that research will still be fun in the future. John, if at my semi-retirement, I can have the same excitement that you have for your work in the lab and the classroom then I will know my career has been a success. Michelle, you have done so much for me that I can never thank you enough for the years of support. And Jim, thank you for giving me the chance. My life was forever changed the day I joined the lab. For that, I am truly grateful. Thank you all for your patience and wisdom. You are all true teachers.

Thank you to all of my friends, especially the couch owners. I never would have done this without the Brightside gang who fueled discussion and helped create and shape ideas. I am also grateful that you all married insightful women who have been able to maintain a sense of intellectual curiosity and ideology while you become more set in your ways. There is hope for your children.

To my family, thank you for the support and the genes. Diversity is a good thing and the Rineharts, Hawkins, Worleys and Smiths (extended Smith clan included) each offer unique perspectives that make life interesting and fun. I truly appreciate the support and guidance that all of you provide. To my parents, thank you. You allowed me to learn for myself and you picked me up when I fell. I can't think of a better way to teach someone how to navigate the experience of life. Everything that I have accomplished is because of you. You have done a terrific job.

To Amelia, I love you. You have not wavered in your support and devotion. I am a lucky man and I can't wait to experience the rest of our journey together. I have found my perfect fit in you. Thank you PP. 


\section{Table of Contents}

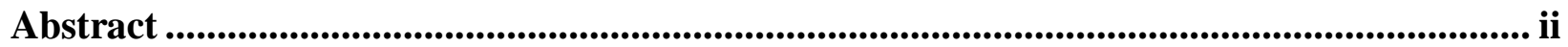

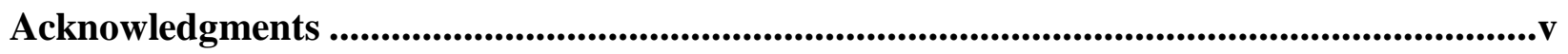

Table of Contents ..................................................................................................................... vi

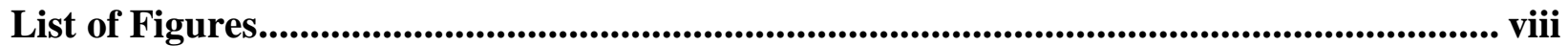

List of Symbols, Abbreviations or Nomenclature ..............................................................

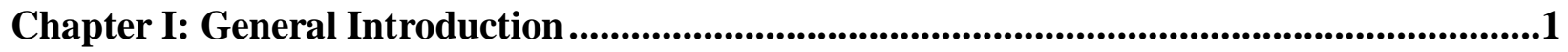

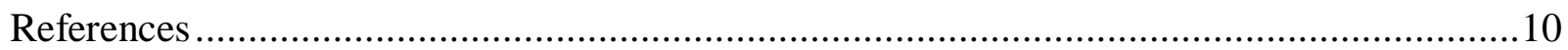

Chapter II: Characterization of morphology and walking kinematics of crayfish

(Procambarus clarkii) .........................................................................................16

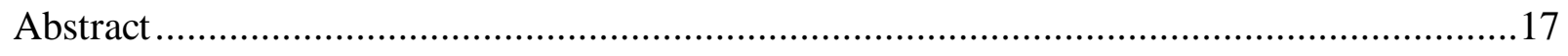

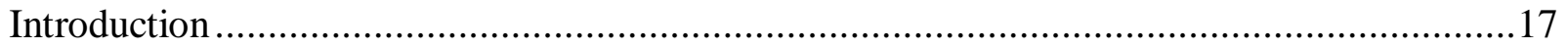

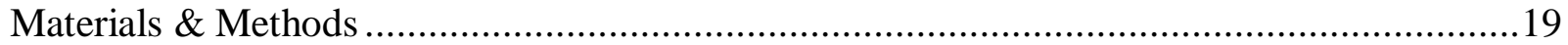

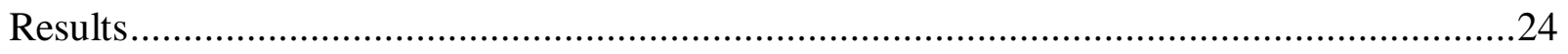

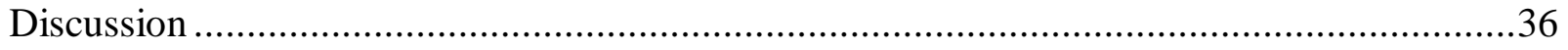

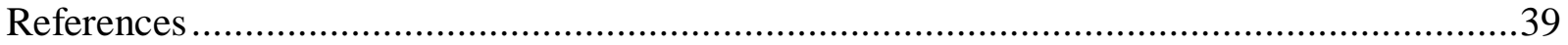

Chapter III: Functionally similar locomotor networks produce flexible walking behavior during crayfish ontogeny ..........................................................................................44

Abstract

Chapter IV: Kinematic analysis of crayfish walking suggests a mechanism for adapting to differential loads........................................................................................................68

Abstract. 


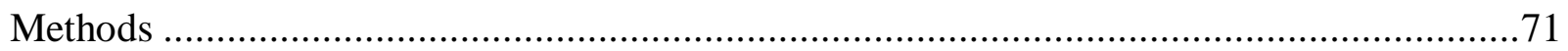

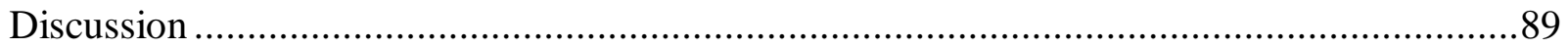

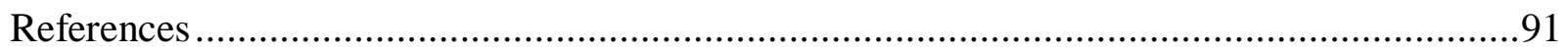

\section{Chapter V: Modeling crayfish locomotion in a real-world environment reveals walking as}

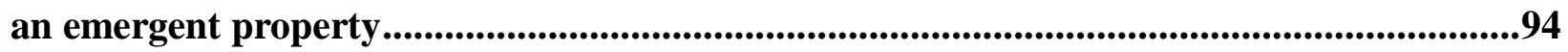

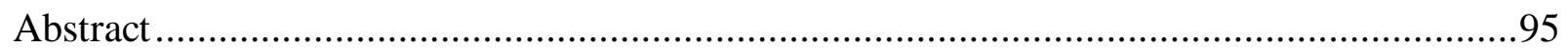

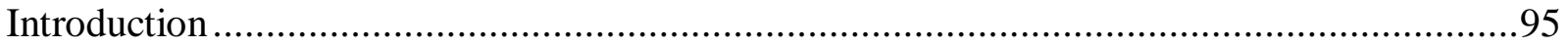

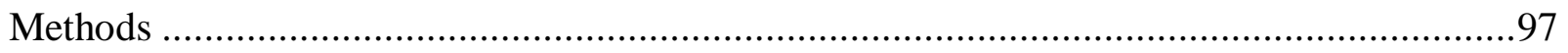

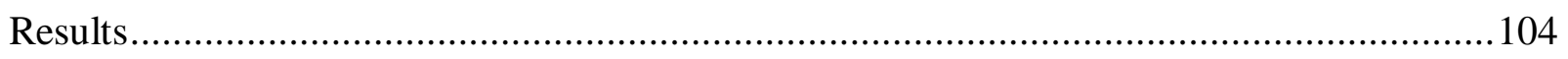

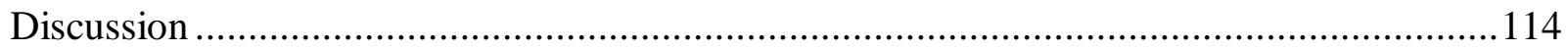

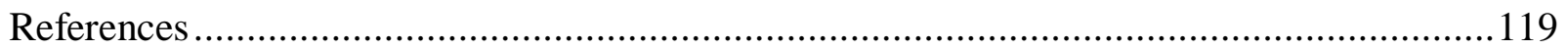

Chapter VI: Summary and Conclusions .......................................................................125

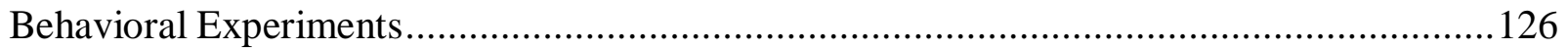

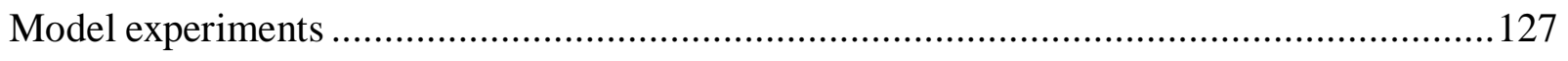

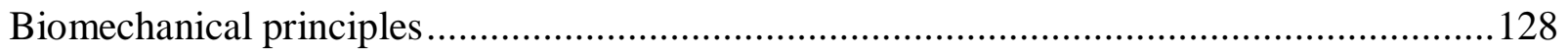

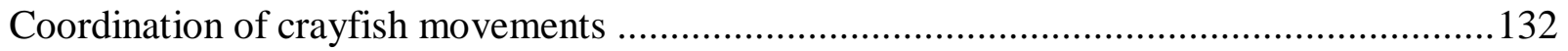

Control mechanisms: Neural vs. Mechanical, Slow vs. Fast ....................................... 133

Comparison to other locomotor systems and evolutionary impact ................................136

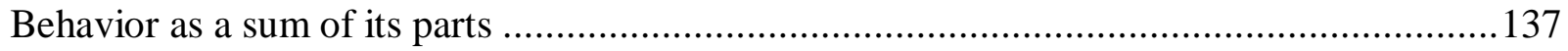

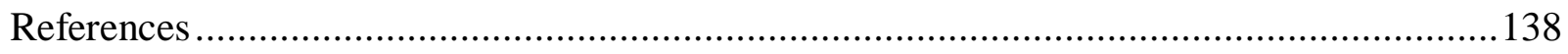

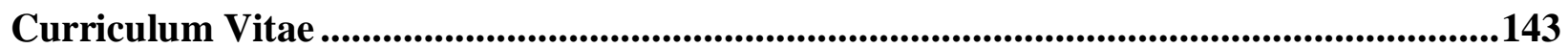




\section{List of Figures}

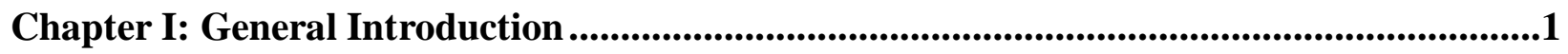

Figure 1.1 Idealized gaits of multi-legged walkers ..................................................

Chapter II: Characterization of morphology and walking kinematics of crayfish

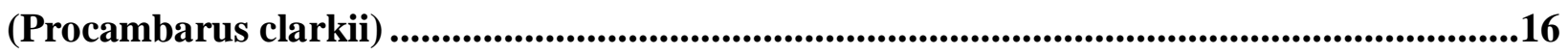

Figure 2.1 Diagram of a juvenile crayfish.............................................................2

Figure 2.2 Allometric relationships of limb and body lengths in Procambarus clarkii......................25

Figure 2.3 Gait diagram of freely walking juvenile crayfish ........................................27

Figure 2.4 Phase relationships between neighboring legs ..............................................29

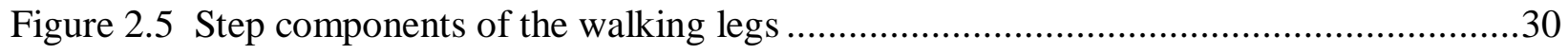

Figure 2.6 Correlation between stance/swing duration and period in the walking legs .............32

Figure 2.7 Kinematics of four joints in each of the walking legs .......................................34

Figure 2.8 Angular velocities of the joints in each of the walking legs ................................35

Chapter III: Functionally similar locomotor networks produce flexible walking behavior

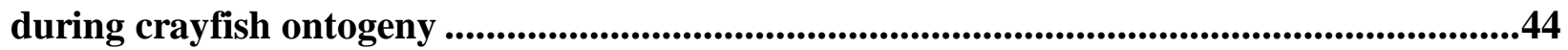

Figure 3.1 Effects of walking speed on ipsilateral phase relationships ................................51

Figure 3.2 Effects of walking speed on components of the step cycle ...............................52

Figure 3.3 Correlations between stance/swing duration and period are

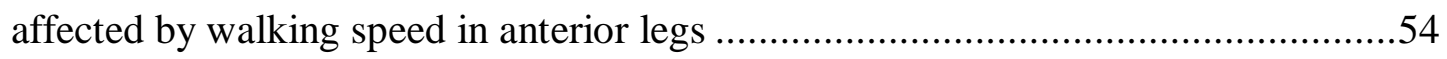

Figure 3.4 Ipsilateral phase relationships after amputation of one and two legs .....................56

Figure 3.5 Effects of limb amputation on step components of stumps and intact legs ..............57

Figure 3.6 Leg amputation affects the correlation between stance duration and period.............59

Figure 3.7 Gait diagrams after amputation of one then two adjacent legs ............................61

Chapter IV: Kinematic analysis of crayfish walking suggests a mechanism for adapting to differential loads..........................................................................................................68

Figure 4.1 Walking velocities of adult and juvenile crayfish after differential loading ............75

Figure 4.2 Step components of adult and juveniles with different centers of mass ..................77 
Figure 4.3 Spatial and temporal components of the stepping profile of each leg in adults and juveniles before and after differential loading .79

Figure 4.4 Effects of center of mass location on ipsilateral phase relationships .....................82

Figure 4.5 Effects of center of mass location on contralateral phase relationships ..................83

Figure 4.6 Phase relationships describing interlimb coordination of the chelae ......................84

Figure 4.7 Example of joint kinematics during freely walking in adults .............................86

Figure 4.8 Mean angular excursions of four joints before and after shifting the center of mass .87

Figure 4.9 Mean angular velocities of four joints before and after shifting the center of mass ...88

\section{Chapter V: Modeling crayfish locomotion in a real-world environment reveals walking as}

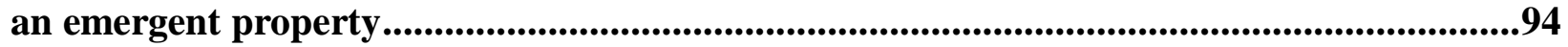

Figure 5.1 Diagram of the model crayfish body plan .................................................... 99

Figure 5.2 Diagram of the neural circuitry controlling movement in individual legs ..............101

Figure 5.3 Phase relationships describing interlimb coordination in the model

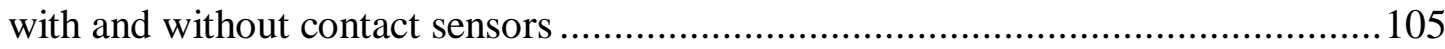

Figure 5.4 Effects of contact sensors on step components .......................................... 106

Figure 5.5 Center of mass location affects ipsilateral phase relationships similar to real animals 108

Figure 5.6 Center of mass location affects contralateral phase relationships......................... 109

Figure 5.7 Step components in the model change based on proximity to the center of mass ....110 Figure 5.8 Leg amputations result in new interlimb coordination between intact legs ............112

Figure 5.9 Gait diagrams of model crayfish compared to actual crayfish

Figure 5.10 Mechanical influence of a rigid bodied system on potential interlimb coordinating mechanisms .115

Figure 5.11 Static instability during walking influences stepping patterns of the legs 118

Chapter VI: Summary and Conclusions 125

Figure 6.1 Crayfish body trajectory during walking resembles an inverted pendulum 129

Figure 6.2 Contralateral phase relationships are correlated in neighboring leg pairs 


\section{List of Symbols, Abbreviations or Nomenclature}

\begin{tabular}{|c|c|}
\hline AEP & Anterior extreme position \\
\hline Astacus & Astacus leptodactylus \\
\hline $\mathrm{CB}$ & Coxo-basiopodite joint \\
\hline $\mathrm{CBCO}$ & Coxo-basal chordotonal organ \\
\hline CNS & Central nervous system \\
\hline $\mathrm{CO}$ & Chordotonal organ \\
\hline $\mathrm{COM}$ & Center of mass \\
\hline $\mathrm{CP}$ & Carpo-propodite joint \\
\hline CPG & Central pattern generator \\
\hline CSD & Cuticular stress detector \\
\hline $\mathrm{FCO}$ & Funnel canal organ \\
\hline IM & Ischio-meropodite joint \\
\hline L1, (L2-L5) & Left leg 1 , left legs $2-5$ \\
\hline $\mathrm{MC}$ & Mero-carpopodite joint \\
\hline $\mathrm{MCO}$ & Myochordotonal organ \\
\hline $\mathrm{MRO}$ & Muscle receptor organ \\
\hline $\mathrm{P}$ & Period \\
\hline P. clarkii & Procambarus clarkii \\
\hline PEP & Posterior extreme position \\
\hline Procambarus & Procambarus clarkii \\
\hline R1, (R2-R5) & Right leg 1 , right legs $2-5$ \\
\hline $\operatorname{Re}$ & Reynolds number \\
\hline $\mathrm{TC}$ & Thoraco-coxopodite joint \\
\hline TCCO & Thoraco-coxal chordotonal organ \\
\hline TCMRO & Thoraco-coxal muscle receptor organ \\
\hline
\end{tabular}


Chapter I: General Introduction 
Whether flying, swimming, gliding, walking, crawling, or slithering, animals must obey rules prescribed by their surroundings using only the tools evolution has allowed. This does not mean that the toolbox is limited. Animals have acquired seemingly endless various forms and functions to solve that life-long problem - how to get from point A to point B. Body morphologies - the interfaces between organism and environment - bear out the compromises made throughout evolutionary histories. Muscle design and performance work to generate the forces required for movement. Nervous systems link the environment and muscles by signaling what is and what needs to be done. Ultimately, what arises from the pieces is simply locomotion.

It is easy to think of locomotion as a struggle to overcome forces. An elephant has to overcome its weight to propel itself forward. An earthworm fights against friction. A monarch butterfly battles against air resistance and gravity. It is a fact that animals must expend energy to produce motion. However, we may find that animals are more efficient at locomotion than we anticipate and the environment may be more assisting than we think.

In this thesis, I address only a few of these issues. I use the crayfish, Procambarus clarkii, as a model system to study the effects of hydrodynamics and load distribution on walking. I focus on body morphologies and kinematic analysis of walking behavior of juvenile and adult crayfish to determine how the nervous system produces successful motor output under various conditions. I have synthesized realistic walking behavior in a virtual crayfish to test hypotheses and assumptions that have arisen from behavioral experiments.

Crayfish are decapod crustaceans within the phylum Arthropoda. They are ten-legged, amphibious invertebrates with an exoskeleton. Crayfish are long-bodied decapods of the infraorder Astacidea, which also includes lobsters. The anterior legs are large pincers called chelae, commonly called claws (referenced as leg 1). The next two pairs of legs also have pincers but they are much smaller in size and are referred to as chelipeds (legs 2 and 3). The posterior legs (4 and 5) do not have pincers. Legs 2-5 are commonly referred to as the walking legs because the chelae may or may not be used for locomotion. Each leg has seven segments with seven joints controlled by a pair of antagonistic muscles. All of the legs are attached to the ventral side of the thorax, offset in the caudal direction. The dorsal thorax is composed of three 
fused segments. The ventral thorax is not attached to the dorsal thorax by the exoskeleton, but instead by endothelium and muscle on the inner side of the dorsal thorax. The area between the two exoskeletal bodies, called the brachial groove, houses the gills. With concern to locomotion, the entire thorax acts as a single non-articulating segment of the body. The abdomen of the crayfish has six articulating segments and a tail fan, made of the telson and uropods. The abdomen is largely comprised of muscle and functions primarily in escape and swimming. The uropods have been implicated in stabilizing mechanisms controlled by the statocysts, which are analogous to the mammalian vestibular system (Cohen, 1955). Each segment of the abdomen contains two pairs of appendages known as periopods, or swimmerets. As their name suggests, they are used primarily for swimming, but a recent study on lobsters showed that the swimmerets can produce a small rostral-, or forward-, directed propulsive force that may assist in walking (Lim and DeMont, 2009).

The central nervous system (CNS) of the crayfish is made of a nerve cord that extends from the head, or rostrum, to the caudal end of the abdomen. The nerve cord is anatomically segmented and contains collections of neurons and accessory cells (collectively known as neuroganglia) that are conglomerated in each segment. Each ganglion sends processes to adjacent ganglia and beyond. Each ganglion also controls muscles associated with that area of the body through motor neurons. Sensory neurons receive information about the environment and send inputs to the CNS. Walking is primarily controlled by neurons in the thoracic ganglia. Each of the last five thoracic ganglia are associated with leg pairs 1-5 and send motor neurons to muscle pairs in each leg segment. Furthermore, each ganglion is divided bilaterally into two hemiganglia. Left and right hemiganglia control motor output to left and right legs of that segment.

Neurons involved in locomotion are often called the walking circuit, or motor pattern generator. While the walking circuitry may receive inputs from systems outside the thoracic nerve cord, such as statocysts, many animals can produce successful walking behavior without these inputs (Shik and Orlovsky, 1976). Therefore, the walking circuitry is housed within the thoracic nerve cord. This is not unique to crayfish or crustaceans. From arthropods to mammals, many animals produce walking-like output by neural networks located in discrete regions of the CNS separate from the brain (insects, Pearson and Iles, 1973; fish, Grillner, 1974; cats, Shik and Orlovsky, 1976, Grillner and Zangger, 1979). Experiments have shown that many animals can still 
produce successful locomotion when the section of the CNS housing the walking circuitry has been isolated by pharmaceutical techniques or by ablating connectives to the brain (Shik and Orlovsky, 1976).

Anatomically isolated locomotor networks can produce endogenous rhythmic output (Pearson, 1993). Isolated central nervous systems of many animals, including crayfish, can generate rhythmic patterns of activity that can be recorded in the motor neurons that control the muscles used in locomotion (Sillar et al., 1987; Delcomyn, 1980). These oscillating networks of neurons, called central pattern generators (CPGs), can even function in an isolated ganglion (Chrachri and Clarac, 1990). The half center model shows that three cells can produce an oscillating network (Graham Brown, 1914). Two cells that receive tonic, or sustained, excitation from a third cell can reciprocally inhibit one another so that when one cell fires the other is silent. As the excited cell fatigues, the silent cell overcomes the inhibition with the help of the tonic excitation from the third cell. This mechanism, or some close variation, is thought to be used by many animals to produce endogenous rhythmic activity (Kristan and Weeks, 1983; Friesen, 1994). Unfortunately, a complete network architecture for a locomotor CPG has yet to be identified. The closest that neuroethologists have come to this goal is the description of the CPG in the stomatogastric ganglion which controls muscle function of crustacean digestive organs (Miller and Selverston, 1982; Marder and Bucher, 2001).

While a detailed organization of any locomotor nervous system has remained elusive, scientists have been able to map out parts of many systems and quantify function of the portions of locomotor networks. Flying and swimming systems have proved useful in this endeavor because locomotor output may be less variable than in walking systems, especially multi-legged walking. Most swimming CPGs only have one oscillator per body segment (Roberts and Tunstall, 1990). Half of the oscillator controls flexion on one side of the body and the other half oscillator induces flexion of the opposite, or contralateral, side (Roberts and Tunstall, 1990). Therefore, flexion on one side also produces extension on the contralateral side. Reciprocal inhibition occurs across hemiganglia through commissural interneurons (Buchanan, 1999; Soffe et al., 1984; Dale, 1985). Flying requires another level of control because of the presence of an appendage. A wing beat is controlled by at least two antagonistic muscles: an elevator to raise the wing and a depressor to push it down. Therefore, a wing CPG produces oscillations in the 
limb on one side of the body only (Robertson and Pearson, 1985). For successful flight, the two contralateral CPGs must be coordinated. This may be accomplished by commissural interneurons that bilaterally connect hemiganglia in the flight pattern generator (Robertson and Pearson, 1983). Therefore, the role of commissural interneurons would be unique in flying systems (coordinate two oscillators) compared to swimming systems (reciprocally inhibit half centers). Most swimming systems are multi-segmented so coordination between adjacent segmental, or ipsilateral, oscillators is still required (Namba and Mulloney, 1999; Tschuluun et al., 2001).

Walking systems show a great deal more variation in design and function. Locomotor programs appear to be different in vertebrates and invertebrates and this may be correlated with the number of limbs the animal has. Similar to flying systems, each leg has at least one oscillator controlling rhythmic output (it has been suggested that stick insects have individual oscillators for each leg joint; Büschges et al., 1995; Bässler and Büschges, 1998). Four-legged vertebrates appear to use commissural interneurons to coordinate contralateral CPGs (Kjaeruff and Kiehn, 1997). There is also evidence that vertebrates have ipsilaterally coupled CPGs between fore- and hindlimbs on the same side of the body (Goulding, 2009). Such high levels of control may be required by four-legged animals to keep from falling. If the center of mass is located within a base of support provided by the legs, strict coordination may be necessary to provide dynamic stability as the animal walks. Arthropods with 6-10 walking legs may not require as much derived control from the nervous system. More legs mean more potential combinations of legs that can be used as a base of support. Therefore, a programmed stepping pattern, or gait, may not be necessary. Isolated nerve cord experiments in crayfish have suggested connectivity between ipsilateral oscillators, but no coordination between contralateral hemiganglia (Sillar et al., 1987). Semi-intact preparations on stick insects provided similar findings (Borgmann et al., 2007). Unfortunately, these studies only suggest the existence of neural circuitry between ipsilateral oscillators and not a mechanism for actual coordination. In both experiments all ipsilateral oscillators fired in-phase, or in synchrony, with each other (Figure 1.1A). This is contrary to kinematic studies on freely walking arthropods in which legs moved primarily out-of-phase, or alternating, with ipsilateral adjacent legs (Hughes, 1952; Wendler, 1966; Wilson, 1966; Jamon and Clarac, 1995). In many systems it has been suggested that peripheral feedback to the CPG is necessary for behaviorally realistic output at the level of individual oscillators (Robertson and 
Pearson, 1983; Sillar et al., 1986). Sensory feedback appears to be critical for proper coordination of multiple oscillators during arthropod walking (Borgmann et al., 2007). In this dissertation, I test the levels of functional connectedness between ipsilateral and contralateral legs necessary for walking in crayfish by adjusting the level of sensory inputs to the CPGs.

The lack of a well defined locomotor neural network leaves neuroethologists with questions as to how exactly sensory inputs from the environment affect CPGs to produce the observed output. But before we can ask how a nervous system produces behavior, we must know what exactly it is producing. Detailed biomechanical and kinematic analysis of locomotion under a number of experimental conditions is the first step. Kinematic analyses in freely walking arthropods reveal gaits that do not closely resemble activity of the isolated nerve cord (Barnes, 1977; Jamon and Clarac, 1995; Wendler, 1966; Wilson, 1966; Delcomyn, 1971). The actual gaits are often idealized as two alternating units of legs. For example, six-legged walkers typically use an alternating tripod gait in which the first and third ipsilateral legs and the second contralateral leg form a tripod that acts as a single functional unit (Delcomyn, 1971). This tripod alternates swing and stance phases with the other tripod (Figure 1.1B). Eight-legged walkers (Astacidea) under this conceptualized extension would step with an alternating tetrapod gait. When these gaits are diagrammed, they appear to suggest patterned activity in adjacent legs that travels as a metachronal wave along the body in either an ascending or descending coordination (arrows, Figure 1.1C). This has led to hypotheses about metachronal waves of activity which suggest a connectedness between legs (Cruse, 1990). Metachronal coordination is found in the swimmerets on the crayfish abdomen (Tschuluun et al., 2001) and extrapolating a similar hypothesis about leg activity is not far-fetched based on kinematic analyses. However, coordinating mechanisms and neural circuitries are not well defined for any walking system.

In reality, animals display variability in their gait that these idealized models do not show. This appears partially to be a function of leg number (Wendler, 1966; Clarac, 1981). Insects show an alternating tripod gait more so than Astacidea follow the alternating tetrapod model (Wendler, 1966; Clarac, 1981). This may be the result of one of two causes. One, fewer legs limit the possible gaits that can provide stability during walking movements. Two, walking speed may require animals to stereotype gait. The slower an animal walks the more likely multiple legs will be in contact with the substrate at any given time if stance durations are longer than swing 
A

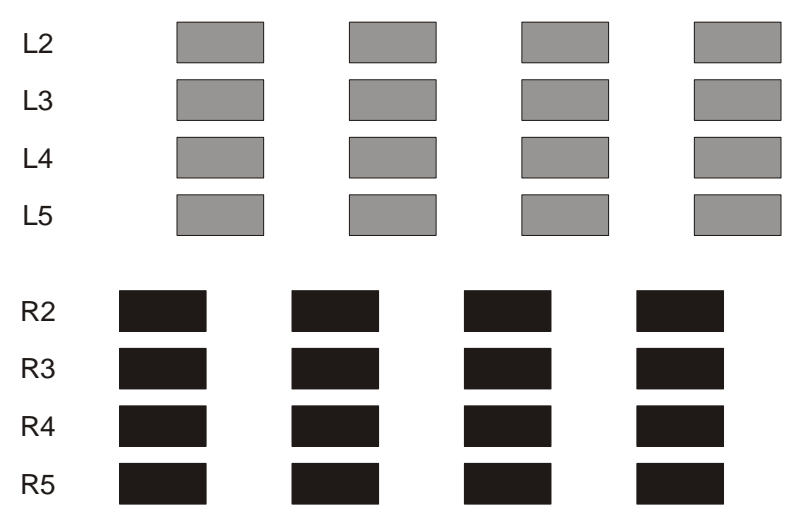

B

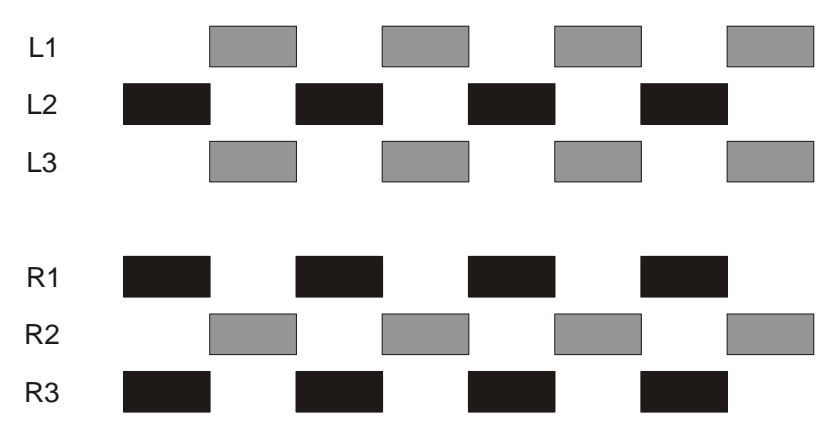

C

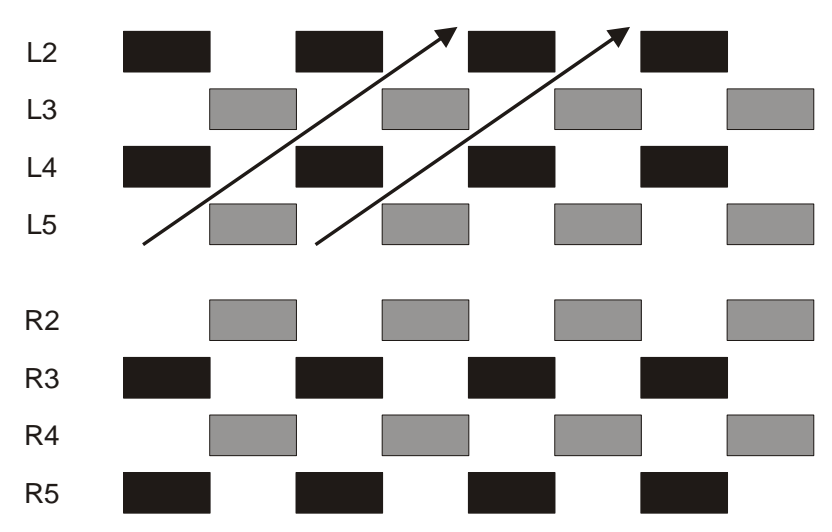

Figure 1.1. Idealized gaits of multi-legged walkers. A) Coordination of central pattern generators with no peripheral feedback is in-phase between ipsilateral hemiganglia. Contralateral hemiganglia show no distinct coordination. Boxes represent power stroke, or stance phase; open phases represent return stroke, or swing phase. Black and gray boxes represent separate functional units. B) Many six-legged walkers use an alternating tripod gait in which legs L2-R1-R3 act as a tripod unit and legs R2-L1-L3 act as the other alternating unit. C) By extension, eight-legged walkers can be said to use an alternating tetrapod gait. Arrows show metachronal waves of activity in the legs. 
durations. Decreasing walking speeds on a treadmill cause increases in stance durations with little or no change to swing duration (Chasserat and Clarac, 1983; Clarac and Chasserat, 1986). Potentially fast walkers such as the cockroach, Periplaneta americana, show gait transitions at different speeds (Full and Tu, 1991). Slower walkers, such as stick insects (Carausius morosus) and Astacidea, do not display gait transitions. Instead, these animals show the same gait across a range of walking speeds. As walking speed increases, coordination becomes less variable (Graham, 1972 Chasserat and Clarac, 1983; Clarac and Chasserat, 1986). These behavioral differences have resulted in the idea that when walking slowly arthropods use complex neural coordinating mechanisms while fast walking is mostly dependent on mechanical reflexes to maintain limb coordination (Full and Koditschek, 1999).

Changing the load on crayfish has a similar effect as changing walking speed. Increased load tends to cause interlimb coordination to become more alternating and less variable (Pond, 1975; Grote, 1981; Clarac and Barnes, 1985). It is known that cuticular stress detectors (CSD) in the proximal leg and funnel canal organs (FCO) at the distal leg respond to load on the leg (Duysens et al., 2000; Klärner and Barnes, 1986). Depending on the type of stimulation, the CSDs provide a reflex response which enhances the activity of the leg (e.g., depressor muscles are excited if a load increases) (Duysens et al., 2000). It is unknown if this reflex pathway has influence on the rest of the leg as in the form of a chained reflex response. It is also unknown if the CSD reflex can directly influence adjacent legs. Activity in the FCO in crabs has been correlated to activity in the depressor muscles of adjacent legs (Libersat et al., 1987). However, the mechanism for the increased output has yet to be described. There may be a direct neural pathway, or the leg being stimulated may change its behavior so that adjacent legs have to adapt. Therefore, large loads may cause a broad change to the entire walking circuit, or alternatively, reflexes may be isolated to individual legs and behavior of the other legs will manifest itself through the original leg's sense organs. Zill et al. (2009) have shown that load transfer to another leg correlates with initiation of swing phase in the "unloaded" leg. This suggests functional coupling between legs that does not require direct connections in the neural circuitry.

The main focus of my research has been to understand how the nervous system produces walking. I have chosen crayfish as a model system to test how nervous systems can accommodate large changes in body size during an ontogeny. The difference in size between 
very young juvenile and mature adult crayfish may mean that hydrodynamic forces act differently on an organism at the beginning of its life. The nervous system also has to accommodate a tremendous change in mass over a lifespan. It is very likely that as a crayfish ages the distribution of load is also different because chelae appear to be relatively larger in adults. Therefore, the walking circuitry also has to deal with load differences on individual legs. All of this is assuming that a functionally similar nervous system exists in these animals.

The first set of experiments that I have conducted, presented in chapter 2, test the effects of size and fluid dynamics that act on the crayfish during locomotion. Juvenile and adult crayfish are very similar in body morphology but they differ greatly in size. The effect of such a large size difference may mean that juvenile crayfish have to deal with a relatively more viscous environment (Vogel, 1994). It is in large part a comparative study meant to quantify walking behavior in juvenile crayfish to compare with previous studies on freely walking adults (Jamon and Clarac, 1995; Jamon and Clarac, 1997) which may walk in a more inertial environment. I also quantify allometric relationships between body and limb morphologies of juvenile and adult crayfish. These size relationships are the basis for potential differences in hydrodynamic influence during walking (Schmidt-Nielsen, 1984; Alexander, 2003; Vogel, 1994).

I next wanted to determine whether differences in walking between juveniles and adults could be attributed to developmental changes in the locomotor circuitry. Therefore, I revisited treadmill experiments on adult crayfish and lobsters in which legs were amputated to reduce sensory feedback to the leg CPGs (Clarac, 1978; Clarac and Chasserat, 1979). I had two aims in performing these experiments on juvenile crayfish: 1) To discover whether amputated stumps show the same activity as adult stumps (which resemble activity of isolated hemiganglia; Sillar et al., 1986) and 2) to see if the remaining intact legs adopt a new coordination. While I had juveniles tethered on the treadmill, I also induced multiple walking speeds to determine if limb coordination changed. The overall goal for the treadmill experiments, presented in chapter 3 , was to determine if juvenile and adult crayfish respond in the same way to similar perturbations.

In the final behavioral experiment, I tested the effects of changing load distribution across the legs. Previous studies have shown that increasing the load on animals, by adding weight or walking out of water, causes more stereotyped, less variable limb coordination in all legs (Clarac 
and Barnes, 1985; Grote, 1981; Pond, 1975). In the experiments presented in chapter 4, I shift the center of mass in juvenile and adult crayfish. My goal is to test whether discrete portions of the locomotor program would change with localized differences in load.

Finally, I build a virtual crayfish using the simulation software AnimatLab that includes a realworld physics engine (Vortex ${ }^{\mathrm{TM}}$ ) to test how the location of center of mass affects leg behavior and interlimb coordination.

\section{References}

Alexander, R. M. (2003). Principles of animal locomotion. Princeton University Press, Princeton, Oxford, pp 53-67

Barnes, W. J. P. (1977). Proprioceptive influences on motor output during walking in the crayfish. J. Comp. Phsiol. (Paris) 73, 543-564

Bässler, U and Büschges, A. (1998). Pattern generation for stick insect walking movements multi-sensory control of a locomotor program. Brain Res. Rev. 27, 65-88

Beer, R.D. (1990). Intelligence as adaptive behavior: an experiment in computational neuroethology. Chandrasekaran, B. (ed.). Academic Press, Inc., San Diego

Borgmann, A., Scharstein, H., and Büschges, A. (2007). Intersegmental coordination: Influence of a single walking leg on the neighboring segments in the stick insect walking system. $J$. Neurophysiol. 98, 1685-1696

Buchanan, J.T. (1999). Commissural interneurons in rhythm generation and intersegmental coupling in the lamprey spinal cord. J. Neurophysiol. 81, 2037-2045

Büschges, A., Schmitz, J., and Bässler, U. (1995). Rhythmic patterns in the thoracic nerve cord of the stick insect induced by pilocarpine. J. exp. Biol. 198, 435-456 
Chasserat, C. and Clarac, F. (1983). Quantitative analysis of walking in a decapods crustacean, the rock lobster Jasus lalandii. II. Spatial and temporal regulation of stepping in driven walking. J. exp. Biol. 107, 219-243

Chrachri and Clarac, F. (1990). Fictive locomotion in the fourth thoracic ganglion of the crayfish, Procambarus clarkii. J. Neurosci. 10(3), 707-719

Clarac, F. (1978). Locomotory programs in basal leg muscles after limb autotomy in the crustacea. Brain Res. 145, 401-405

Clarac, F. (1981). Decapod crustacean leg coordination during walking. In: Locomotion and energetic in arthropods. Herreid, C.F. and Fourtner, C.R. (eds.). Plenum Press, New York, London. pp 31-71

Clarac, F. and Barnes, W. J. P. (1985). Peripheral influences on the coordination of the legs during walking in decapods crustaceans. In: Coordination of motor behavior. Bush, B.M.H. and Clarac, F. (eds.). Cambridge University Press, Cambridge. pp 249-269

Clarac, F. and Chasserat, C. (1979). Experimental modifications of interlimb coordination during locomotion of a Crustacea. Neurosci. Lett. 12, 271-276

Clarac, F. and Chasserat, C. (1986). Basic processes of locomotor coordination in the rock lobster. I. Statistical analysis of walking parameters. Biol. Cybern. 55, 159-170

Cohen, M.J. (1955). The function of receptors in the statocyst of the lobster Homarus americanus. J. Physiol. 130, 9-34

Cruse, H. (1990). What mechanisms coordinate leg movement in walking arthropods? Trends Neurosci. 13, 15-21

Cruse, H., Dürr, V., Schmitz, J. (2007). Insect walking is based on a decentralized architecture revealing a simple robust controller. Phil. Trans. R. Soc. A 365, 221-250

Dale, N. (1985). Reciprocal inhibitory interneurons in the Xenopus embryo. J. Physiol. 363, 61-70 
Delcomyn, F. (1971). The locomotion of the cockroach Periplaneta americana. J. exp. Biol. $54,443-452$

Delcomyn. F. (1980). Neural basis of rhythmic behavior in animals. Science 210, 492-498

Delcomyn, F. (1981). Insect locomotion on land. In: Locomotion and energetic in arthropods. Herreld, C.F. and Fourtner, C.R. (eds.). Plenum Press, New York. pp. 103-125

Duysens, J., Clarac, F. and Cruse, H. (2000). Load-regulating mechanisms in gait and posture: comparative aspects. Physiological Reviews. 80, 83-133

Ekeberg, Ö., Blümel, M., and Büschges, A. (2004). Dynamic simulation of insect walking. Arthropod Struct. Dev. 33, 287-300

Friesen, W.O. (1984). Reciprocal Inhibition: A Mechanism Underlying Oscillatory Animal Movements. Neurosci and Biobehav Rev 18, 547-553

Full, R.J. and Koditschek, D.E. (1999). Templates and anchors: neuromechanical hypotheses of legged locomotion on land. J. exp. Biol. 202, 3325-3332

Full, R.J. and Tu, M.S. (1991). Mechanics of a rapid running insect: two-, four- and six-legged locomotion. J. exp. Biol. 156, 215-231

Goulding, M. (2009). Circuits controlling vertebrate locomotion: moving in a new direction. Nature Reviews Neuroscience. 10, 507-518

Graham, D. (1972). A behavioural analysis of the temporal organization of walking movements in the first instar and adult stick insect (Carausius morosus). J. Comp. Physiol. 81, 23-52

Graham Brown, T. (1914). On the nature of the fundamental activity of the nervous centres; together with an analysis of the conditioning of rhythmic activity in progression, and a theory of the evolution of function in the nervous system. J. Physiol. 48, 18-46

Grillner, S. (1974). On the generation of locomotion in the spinal dogfish. Exp. Brain Res. 20, $459-470$ 
Grillner, S. and Zangger, P. (1979). On the central generation of locomotion in the low spinal cat. Exp. Brain Res. 34, 241-261

Grote, J. R. (1981). The effect of load on locomotion in crayfish. J. exp. Biol. 92, 277-288

Hughes, G.M. (1952). The co-ordination of insect movements. I. The walking movements of insects. J. exp. Biol. 29, 267-284

Jamon, M. and Clarac, F. (1995). Locomotor patterns in freely walking crayfish (Procambarus clarkii). J. exp. Biol. 198, 683-700

Jamon, M. and Clarac, F. (1997). Variability of leg kinematics in free-walking crayfish, Procambarus clarkii, and related inter-joint coordination. J. exp. Biol. 200, 1201-1213

Kjaerulff, O. and Kiehn, O. (1997). Crossed rhythmic synaptic input to motoneurons during selective activation of the contralateral spinal locomotor network. J. Neurosci. 17, 9433-9447

Klärner, D. and Barnes, W. J. P. (1986). The cuticular stress detector (CSD2) of the crayfish. II. Activity during walking and influences on leg coordination. J. exp. Biol. 122, 161-175

Kristan, W.B. and Weeks, J.C. (1983). Neurons controlling the initiation, generation, and modulation of leech swimming. Symp. Soc. Exp. Biol. 37, 243-260

Libersat, F., Clarac, F. and Zill, S. N. (1987a). Force-sensitive mechanoreceptors of the dactyl of the crab: single-unit responses during walking and evaluation function. J. Neurophysiol. 57, $1618-1637$

Lim, J.L. and DeMont, M.E. (2009). Kinematics, hydrodynamics and force production of pleopods suggest jet-assisted walking in the American lobster (Homarus americanus). J. exp. Biol. 212, 2731-2745

Namba, H. and Mulloney, B. (1999). Coordination of limb movements: three types of intersegmental interneurons in the swimmeret system, and their responses to changes in excitation. J. Neurophysiol. 81, 2437-2450 
Marder, E. and Bucher, D. (2001). Central pattern generators and the control of rhythmic movements. Curr. Biol. 11, R986-R996

Miller, J.P. and Selverston, A.I. (1982). Mechanisms underlying pattern generation in lobster stomatogastric ganglion as determined by selective inactivation of identified neurons. II. Oscillatory properties of pyloric neurons. J. Neurophysiol. 48, 1378-1391

Müller, U. and Cruse, H. (1991a). The contralateral coordination of walking legs in the crayfish Astacus leptodactylus. I. Experimental results. Biol. Cybern. 64, 429-436

Pearson, K.G. and Iles, J.F. (1973). Nervous mechanisms underlying intersegmental coordination of leg movements during walking in the cockroach. J. exp. Biol. 58, 725-744

Pearson, K.G. (1993). Common principles of motor control in vertebrates and invertebrates. Annu Rev. Neurosci. 16, 265-297

Pond, C. M. (1975). The role of the 'walking legs' in aquatic and terrestrial locomotion of the crayfish Austropotambius pallipes (Lereboullet). J. exp. Biol. 62, 447-454

Roberts, A. and Tunstall, M.J. (1990). Mutual re-excitation with post-inhibitory rebound: a simulation study of the mechanisms of locomotor rhythm generation in the spinal cord of Xenopus embryos. Eur. J. Neurosci. 2, 11-23

Robertson, R.M. and Pearson, K.G. (1983). Interneurons in the flight system of the locust: distribution, connections, and resetting properties. J. comp. Neurol. 215, 33-50

Robertson, R.M. and Pearson, K.G. (1985). Neural circuits in the flight system of the locust. J. Neurophysiol. 53, 110-128

Sillar, K.T., Skorupski, P., Elson, R.C., Bush, B.M.H. (1986). Two identified afferent neurons entrain a central locomotor rhythm generator. Nature. 323, 440-443

Sillar, K. T., Clarac, F. and Bush, B. M. H. (1987). Intersegmental coordination of central neural oscillators for rhythmic movements of the walking legs in crayfish. Pasifastacus leniusculus. J. exp. Biol. 131, 245-264 
Schmidt-Nielsen, K. (1984). Scaling: why is animal size so important? Cambridge University Press, New York, NY.

Shik, M.L. and Orlovsky, G.N. (1976). Neurophysiology of locomotor automatism. Physiol. Rev. 56, 465-501

Soffe, S.R., Clarke, J.D.W., and Roberts, A. (1984). Activity of commissural interneurones in the spinal cord of Xenopus embryos. J. Neurophysiol. 51, 1257-1267

Tschuluun, N., Hall, W.M., Mulloney, B. (2001). Limb movements during locomotion: Tests of a model of an intersegmental coordinating circuit. J. Neurosci. 21(19), 7859-7869

Wendler, G. (1966). The co-ordination of walking movements in arthropods. Symp Soc Exp Biol. 20, 229-249

Wilson, D. M. (1966). Insect walking. Annu Rev Entomol. 11,103-151

Vogel, S. (1994). Life in moving fluids. The physical biology of flow. Princeton University Press, Princeton, pp. 81-105

Zill, S.N., Keller, B.R., Duke, E.R. (2009). Sensory signals of unloading in one leg follow stance onset in another leg: Transfer of load and emergent coordination in cockroach walking. $J$. Neurophsiol. 101, 2297-2304 
Chapter II: Characterization of morphology and walking kinematics of crayfish (Procambarus clarkii) 


\begin{abstract}
Crayfish (Procambarus clarkii) experience an almost 20-fold increase in length and a 20,000fold increase in mass over the course of one lifespan. It is unknown if only changing the size of an animal can cause changes in locomotor strategies. Juveniles and adults are structurally similar with respect to body segments and the posterior four pairs of thoracic appendages (the walking legs); the only exceptions are the first thoracic appendages, or chelae. Therefore, crayfish are a good model system to test how a large change in size affects walking behavior. In this study juveniles were observed walking freely. The juveniles showed distinct differences in stepping behavior in anterior (legs 2 and 3) and posterior legs (4 and 5). The last two pairs of legs showed strong alternating coordination in adjacent ipsilateral and contralateral neighboring legs while coordination in the second and third legs was more variable. Step periods, stance durations, and stride lengths were longer in the posterior two pairs of legs also. Furthermore, kinematic analysis of leg joints showed larger angular displacements of the joints in the posterior legs. These findings suggest that juvenile crayfish predominantly walk with the posterior legs while anterior legs may function in an accessory role.
\end{abstract}

\title{
Introduction
}

All animal locomotion is an integrative process between organism and environment. This relationship is relative to every individual organism in its specific environment. Size is one variable that can be affected by environmental forces acting on the body of the organism. Extremely large differences in body size such as those observed in juvenile and adult crayfish may result in animals of either extreme to be exposed to relatively different fluid dynamic environments. The Reynolds number $(R e)$ is a unitless scaling variable that describes relative fluid dynamic environments based on linear size and flow. Low Re represent relatively viscous fluid environments and high $R e$ suggest fluid environments subjected to higher inertia (Alexander, 2003; Vogel, 1994). Based on the amount of growth they display, juvenile crayfish may reside in relatively viscous environments while adult crayfish may live in a world more dominated by inertial factors. One goal of this study was to determine if different size crayfish use different strategies to produce walking behavior. 
Walking behavior in arthropods has been well studied especially in insects (reviews by Wilson, 1966; Wendler, 1966; Ritzmann and Büschges, 2007; stick insect, Cruse, 1976; cockroach, Delcomyn, 1971) and crustaceans (reviews by Clarac, 1981; Clarac and Barnes, 1985; Clarac, 2000). Within the crustaceans much work has focused on walking behavior in the long-bodied Astacidae and Nephropidae (crayfish, Pond, 1975; Barnes, 1977; Grote, 1981; Jamon and Clarac, 1995; lobsters, Homarus americanus, Macmillan, 1975; Ayers and Davis, 1977) and Palinuridae (Jasus lalandii, Chasserat and Clarac, 1980; Clarac and Chasserat, 1983; Chasserat and Clarac, 1983; Palinurus vulgaris, Ayers and Clarac, 1978), as well as the brachyuran crabs (Burrows and Hoyle, 1973; Barnes, 1975). One of the distinguishing characteristics of the astacids and nephropids is the size of the first thoracic appendages, or the chelae. Crayfish and nephropid lobsters have massive chelae with articulating pincers that are typically larger relative to body size than the chelae of palinurids (Macmillan, 1975; Clarac, 1981). For the most part, locomotion by either group does not require the chelae and previous studies focus mainly on the contributions made by the last four pairs of thoracic appendages, or walking legs (Clarac, 1981).

Crayfish usually display an alternating coordination between adjacent ipsilateral legs (Jamon and Clarac, 1995). It has been suggested that there may be neural signaling between adjacent ipsilateral legs that contributes to coordination in stick insects (Ludwar et al., 2004) and locusts (Laurent, 1986; Laurent and Burrows, 1989b). No such signal pathways have been directly identified in aquatic walking crayfish or lobsters. However, rhythmic activity in isolated thoracic nerve cords of crayfish suggests that it is possible that similar neural connectivity exists (Sillar et al, 1987). In the absence of sensory feedback the resulting activity is synchronous between adjacent ipsilateral legs. These results are contrary to walking coordination in the whole animal in which ipsilateral adjacent legs show predominantly alternating coordination (Hughes, 1952; Cruse, 1990; Cruse et al., 1998). Contralateral legs show more variability in their coordination (Müller and Cruse, 1991 a,b). When freely walking, crayfish can display either in-phase (synchronous) or out-of-phase (alternating) coordination in contralateral pairs of legs (Jamon and Clarac, 1995). However, increasing the load on the crayfish causes a more predominantly alternating pattern in contralateral coordination (Barnes in Clarac and Barnes, 1985; Grote, 1981). 
Components of the step cycle in individual legs are also affected by imposed load. Under normal conditions of unrestrained walking in adult P. clarkii, step cycles are fairly constant in all legs (Jamon and Clarac, 1995). Increasing load on crayfish underwater causes legs to decrease cycle frequency, increasing the duration of time spent on the substrate in stance phase (Barnes, 1977). Terrestrial walking also causes an increase in load on crayfish with estimates varying across species (4 to 6-fold in Austropotamobius pallipes, Pond, 1975; 10-fold in Astacus leptodactylus, Klärner and Barnes, 1986; up to 15-fold reported in Procambarus clarkii, Grote, 1981). When walking on land, crayfish show increases in each of the step variables: period, stance duration, and stride length (Pond, 1975; Grote, 1981). Therefore, it may be possible that an increase in body mass as a crayfish grows could influence its walking behavior.

The purpose of this study is to report allometric relationships between juvenile and adult crayfish and describe unrestrained forward walking behavior in the juveniles. The joint kinematics of all four walking legs (2-5) during juvenile walking is also characterized here.

\section{Materials \& Methods}

Juvenile crayfish (Procambarus clarkii, two weeks old) were obtained from the Louisiana State University Aquaculture Center (Baton Rouge, Louisiana, United States of America) and kept in 5 liter plastic tubs. Water temperatures were maintained at $23^{\circ} \mathrm{C}$. Crayfish were fed hermit crab food and allowed to grow normally for 1-2 weeks. Animals that had all of their legs intact and that were large enough to be seen clearly through video cameras were then isolated in smaller trays $(3 \mathrm{~cm} \times 3 \mathrm{~cm} \times 2 \mathrm{~cm})$ until used.

\section{Body and leg segment measurements}

Body and leg segment lengths were measured for twenty-nine Procambarus clarkii ranging in size from $0.7 \mathrm{~cm}$ to $12.1 \mathrm{~cm}$ total body length (measured from the anterior tip of the rostrum to the caudal edge of the telson) and $0.0033 \mathrm{~g}$ to $63.8 \mathrm{~g}$ body mass. The smallest crayfish were captured at 14-28 days after hatching and all showed the ability to walk. All limb measurements were 
made on the dorsal side of the respective segment. The thoraces were measured from the tip of the rostrum to the caudal end of the thorax along the dorsal midline. The abdomen was fully extended and measured from the anterior edge of the first abdominal segment to the caudal end of the telson.

The crayfish leg is comprised of seven segments each of which has a joint proximal to that segment (Figure 2.1). The range of motion of a given joint is mono-planar and orthogonal to its adjacent joints. Limb measurements were obtained for the five most distal segments (ischiopodite, meropodite, carpodite, propodite, and dactyl; Figure 2.1) which account for the majority of leg length. Propodite lengths for the chelipeds (legs 1-3 containing pincers) were measured from the carpo-propodite joint to the dactyl.

\section{General procedure for freely walking experiments}

Forward walking patterns of eight unrestrained juvenile $P$. clarkii, were observed in a small aquarium $(7.8 \mathrm{~cm} \times 2.2 \mathrm{~cm} \times 2.2 \mathrm{~cm})$. Animals were filmed in one hour increments while freely walking inside the aquarium. Successful trials consisted of at least two uninterrupted steps in a straight line that did not include a starting or stopping step. A straight line qualified as no deviation greater than $15^{\circ}$ from the starting direction.

\section{Filming procedure and kinematic model}

Two groups of four crayfish were filmed on two different substrates. The four animals in group one $(0.8 \pm 0.03 \mathrm{~cm}$ mean body length) were filmed on a rubber textured substrate (12 trials, 436 steps). This group was filmed from overhead with one stationary camera (Canon ZR60). The four animals in the second group (ranging in size from 1.4-1.65 cm) were filmed on a transparent layer of cured silicone (Dow Corning, Sylgard ${ }^{\mathrm{TM}}$ ). This group was filmed from overhead with two stationary cameras. For these trials the aquarium was placed on a stage over a light source between two polarized filters (method adapted from Full et al., 1995). The filters caused body and leg segments to illuminate, while the soft cuticle at the joints remained dark thereby giving 


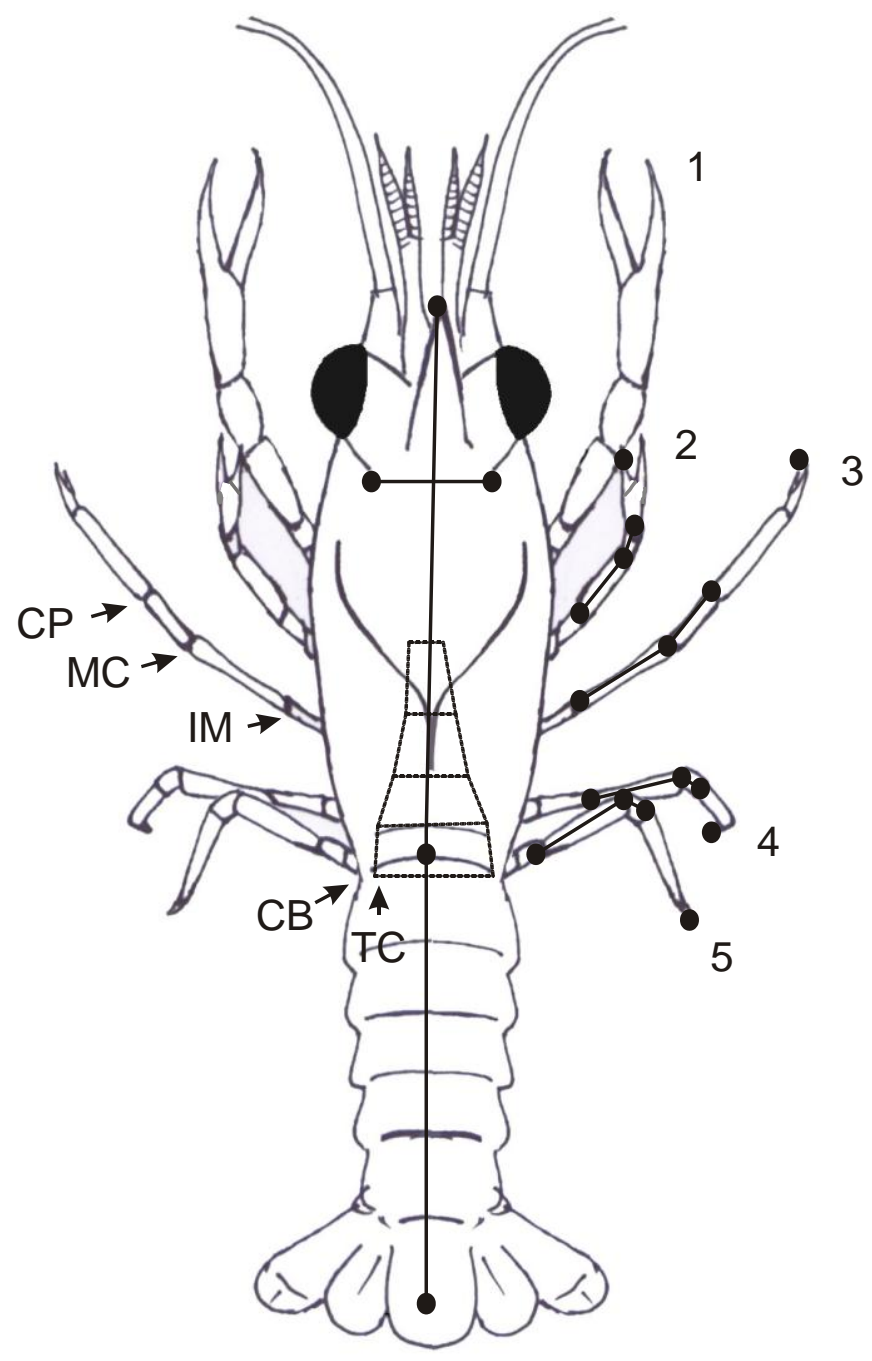

Figure 2.1. A juvenile Procambarus clarkii. The arrows indicate those joints digitized and measured for analysis (thoraco-coxopodite, TC; coxo-basipodite, CB; ischio-meropodite, IM; mero-carpopodite, MC; carpo-propodite; CP). The black dots indicate the positions tracked in the kinematic analysis software. The dashed lines represent a framework of the approximate ventral locations of leg-thorax insertion points. The TC joints are located at the intersections of the dashed lines. 
consistently identifiable markings on the legs (15 trials, 378 steps; Figure 2.1). The longitudinal body axis was manually digitized and defined by three points: 1) the anterior tip of the rostrum;

2) the caudal edge of the thorax at the midline, which was identified by the intestinal tract; and 3) the caudal region of the tail fan (also identified as a dark region surrounded by illuminated muscle in the telson). The soft cuticle at the IM, MC, and CP joints for each leg were all manually digitized when visible in both cameras. The base of each eye stalk was also highlighted through the polarized filters and digitized to give a reference plane with respect to the dorsal carapace. Footfalls and stepping events were digitized using 3-D motion analysis software (Peak Motus) at a frequency of 60 frames s$^{-1}$. All camera fields-of-view were spatially calibrated before each filming session. The field-of-view had dimensions: $30.3 \mathrm{~mm} \times 20.6 \mathrm{~mm}$. The spatial resolution per frame was 720 x 480 pixels. Peak Motus calculated raw threedimensional coordinates which were then used for kinematic analyses in MatLab version 7.0.1.

\section{Joint angle analysis}

Kinematic measurements of the rostro-caudal articulating joints TC and IM as well as the dorsoventral articulating joints $\mathrm{CB}$ and $\mathrm{MC}$ were obtained. The small size of juveniles prevented accurate measurements from isolated TC joints. Therefore, measurements were made of the combined angles of the TC and IM joints. These measurements are presented as excursion angles in the rostro-caudal plane of the proximal leg. These angles were measured by Peak Motus and defined as the angle between the meropodite (IM to MC) relative to the longitudinal axis of the thorax (rostrum to caudal thorax). The CB joint angle was generally defined as the angle between the meropodite and the thorax in the vertical plane. Because the base of the eyes is fixed with the thorax, the angle was measured as the vertical displacement of the meropodite relative to a transverse line between the bases of the eye stalks (see Figure 2.1). This measurement insured accurate dorso-ventral displacement relative to the animal even during possible body roll and yaw displacements. However, severe pitch displacements could reduce resolution of this measurement. If an animal were to pitch severely away from vertical, the result would be a smaller measured CB angle. The animals were not observed to display such drastic 
pitch movements because CB joint angle ranges coincided with those observed in adults (Jamon and Clarac, 1997). The MC joint angle was measured directly.

\section{Treatment of data}

Size and scaling relationships are described by the allometric equation: $y=a x^{b}$, where $x$ and $y$ represent mass and length. Allometric relationships are often exponential and, therefore, most often represented logarithmically (Alexander, 2003). Taking the logarithm of the above equation yields: $\log y=\log a+b \log x$, where $b$ is the slope of the line generated by the equation. It is common form to represent an allometric relationship as simply the independent variable to the power of the slope, $x^{b}$. Therefore, allometric relationships of crayfish body mass to body length are presented as simply $M^{b}$, where $M$ represents the mass of the animal (for further review see Alexander, 2002).

Phase relationships were calculated for all legs using the timing of stance onsets. Phase values were defined as the time of the onset of stance phase in a given leg A, (stance onset $\mathrm{A}$ ) relative to the period (total step cycle duration defined by the time between successive stance onsets) of the reference leg $\mathrm{B}\left(P_{\mathrm{B}}=\right.$ stance onset $\mathrm{n}_{\mathrm{n}+1}-$ stance onset $\left._{\mathrm{n}}\right)$. Therefore, the calculation for the phase value $(\Phi)$ of $\operatorname{leg}_{\mathrm{A}}$ relative to $\operatorname{leg}_{\mathrm{B}}$ is: $\Phi_{\mathrm{A}}$ in $\mathrm{B}=\left(\right.$ stance onset ${ }_{\mathrm{A}}-$ stance onset $\left._{\mathrm{B}}\right) / P_{\mathrm{B}}$. Phase values were calculated for all legs relative to their ipsilateral anterior adjacent neighbors as well as their contralateral segmental counterparts.

Gait diagrams were constructed by referencing stance and swing onsets of all legs to the stance onset time of the right fifth leg (R5). For each R5 step, three variables in all other legs were

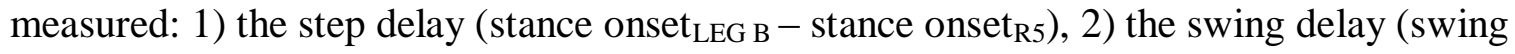
onset $_{\text {LEG B }}$ - stance onset ${ }_{\text {R5 }}$ ), and 3) the period of Leg B. All variables were normalized to the period of that particular step in R5. Data were analyzed as circular data sets due to the cyclic function of a step. All averages were recorded as the circular mean +/- circular standard deviation (c.s.d.) (Fisher, 1993). The initial step of all legs was plotted as the mean normal step delay. Subsequent steps were plotted base on the normalized periods relative to R5. All stance phases were plotted as the duration between mean stance and swing onsets. 
All step variables were calculated using the temporal and spatial markers described above.

Stride lengths were measured as the distance between posterior extreme position (PEP) coordinates and the coordinates of the next anterior extreme position $(A E P)$ in that leg after one swing phase. Swing ratios were calculated as the percentage of a given step that is occupied by

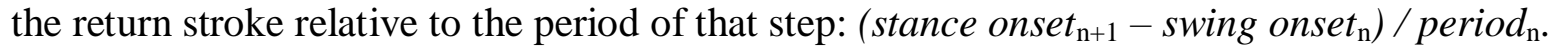
Stance durations were calculated as the absolute time that a dactyl remained in contact with the

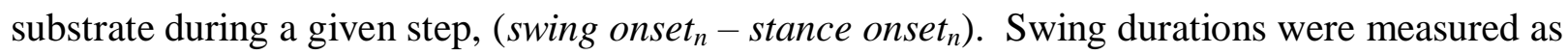

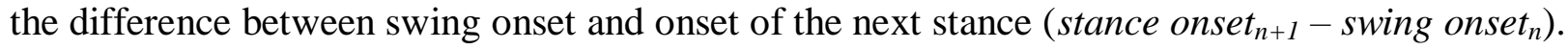
One-way ANOVAs were used for statistical comparison of step variables between legs. Linear regressions were used to identify correlations between stance and swing durations relative to step period. All correlations are expressed as $\mathrm{r}^{2}$ values.

Joint angles and angular velocities were conditioned in Peak Motus using the Butterworth method with a cutoff frequency of $6 \mathrm{~Hz}$. All steps were normalized to their period and joint angles and velocities were interpolated over the normalized step (similar to methods used by Jamon and Clarac, 1997). Data at each time point was then averaged for each animal and all trials. These data are presented as the mean \pm s.e.m.

\section{Results}

A comparison of size ranges between the smallest and largest animals in this study shows a 17fold increase in body length and a nearly 20,000-fold increase in mass (smallest crayfish measured: $3.3 \mathrm{mg}$ with $0.696 \mathrm{~cm}$ body length; largest crayfish measured: $63.8 \mathrm{~g}$ with $12.1 \mathrm{~cm}$ body length).

The eight crayfish walked at velocities that ranged from $0.63 \pm 0.27 \mathrm{~cm} / \mathrm{s}$ to $1.5 \pm 0.31 \mathrm{~cm} / \mathrm{s}$. Normalized to body length, this range was approximately $0.6-1.1$ body lengths/s. 

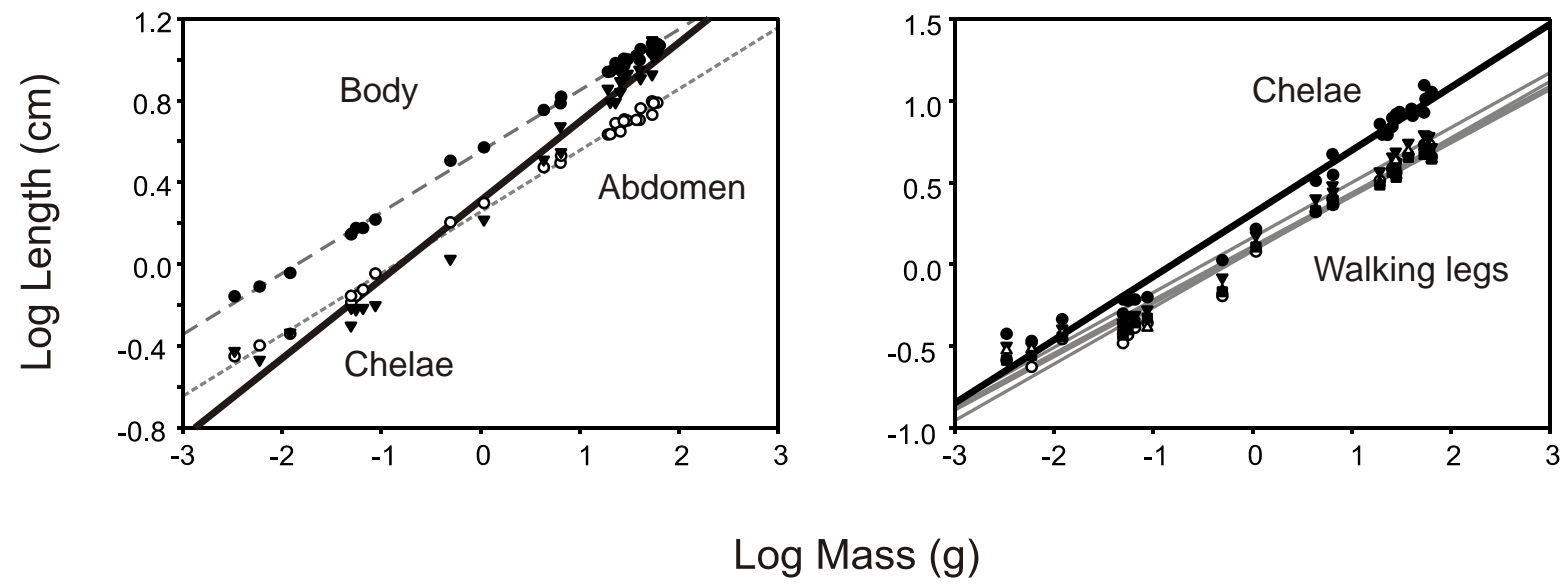

Figure 2.2. Allometric relationships of limb and body lengths in Procambarus clarkii. The chelae, or claws, are disproportionately larger in adults compared to juveniles (chelae: solid black line; abdomen + thorax: long dashed; abdomen: short dashed; walking legs: solid gray). However, all other legs and body segments (abdomen and thorax) scale at a similar ratio (chelae $=\boldsymbol{M}^{\mathbf{0 . 3 9}}$, abdomen $=\boldsymbol{M}^{\mathbf{0 . 3}}$, overall body length $=\boldsymbol{M}^{0.3}$, legs $(2-5)=\boldsymbol{M}^{\mathbf{0 . 3 3}}-\boldsymbol{M}^{\mathbf{0 . 3 5}}$ (B, chelae: filled circles); all adjusted $\left.r^{2}>=0.968\right)$. 


\section{Allometric relationships}

Overall body length increases at a 0.3 ratio relative to the increase in mass $\left(M^{0.3}\right.$, Figure $\left.2.2 \mathrm{~A}\right)$. The parallel increase in body and abdomen length suggests that the thorax and abdomen remain proportionate across sizes and approximately equal in length at any given mass. The chelae grew at a higher rate than the body segments $\left(M^{0.39}\right)$. Juvenile chelae lengths (distance from the BI joint to the dactyl tip) are approximately half the overall body length, closer in length to the abdomen or thorax alone. The largest adult chelae lengths are almost equal to the overall body length, or approximately twice the relative length of juvenile claws. For example, the largest crayfish measured (mass $=63.8 \mathrm{~g}$ ) had a total body length of $12.1 \mathrm{~cm}$ with an average chelae length of $11.3 \mathrm{~cm}$, while the smallest crayfish measured (mass $=3.3 \mathrm{mg}$ ) had a body length of $0.696 \mathrm{~cm}$ and a chelae length of $0.354 \mathrm{~cm}$. The growth rate of the walking legs (2-5) is intermediate to that of the chelae and body lengths $\left(M^{0.33}-M^{0.35} ;\right.$ Fig. 2B). Slopes of the linear regressions of the walking legs were significantly different from those of the chelae and abdomen suggesting that the limbs are relatively longer in adults compared to juveniles with respect to body length. However, the overall increase in the length of the walking limbs is not as large as that of the claws.

\section{Stepping pattern of freely walking juvenile crayfish}

Juvenile crayfish rarely use the chelae during walking. Therefore, descriptions of juvenile walking behavior will only deal with the four pairs of walking legs (thoracic appendages 2-5).

Gait diagrams show the mean stepping pattern of the four pairs of walking legs (Figure 2.3). The most noticeable aspects of the gait diagram are the smaller and more numerous stance boxes in legs 2 and 3. This is a direct result of shorter periods and stance durations. This also suggests high variability in coordination between anterior and posterior legs. Legs 4 and 5 showed longer stance durations and a consistent alternating coordination between ipsilateral neighbors. 


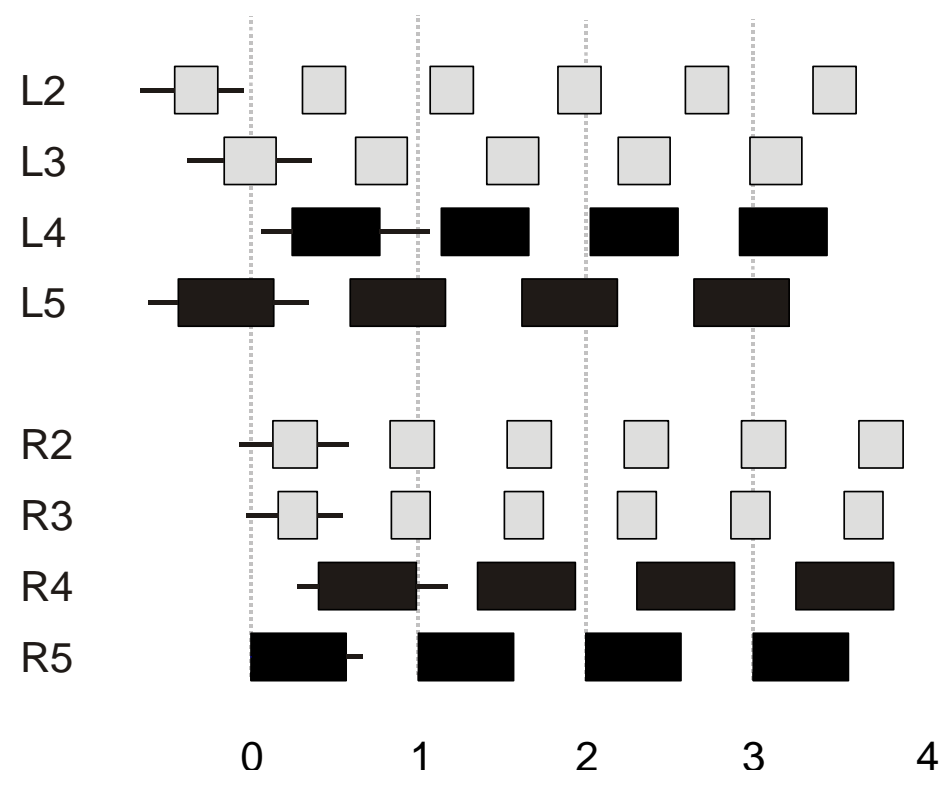

Figure 2.3. Gait diagram of freely walking juvenile crayfish. The stepping pattern is constructed from stance and swing onset times relative to the stance onset time of R5. Period and stance durations are normalized to the step duration of R5 ( $x$-axis, R5 step cycle). The shaded boxes represent stance phase in that particular leg (similar colored boxes highlight the differences between anterior and posterior legs). Error bars represent standard deviation of stance onset time (left side of box) and swing onset time (right side of box). 


\section{Relative coordination between individual legs}

Previous analyses of phase relationships in adult crayfish have shown that ipsilateral adjacent legs are consistently alternating ( 0.5 phase value represents true alternation) while contralateral legs show more variability in coordination (Jamon and Clarac, 1995; Müller and Cruse, 1991a). Juvenile anterior leg pairs 2 and 3 do not maintain any preferred temporal coordination with their contralateral counterparts (Figure 2.4, data for leg 2 not shown). However, contralateral leg pairs 4 and 5 display a distribution with a peak near 0.5 and lower standard deviation suggesting that these legs are predominantly alternating.

Ipsilateral legs 4 and 5 are also alternating as shown by the tight distribution of phase values Figure 2.4). The ipsilateral 5 in 4 coordination is the least variable relationship of all possible interlimb coordinations. Ipsilateral legs, 3 and 4, also displayed a preferred coordination; however, the histogram is more widely distributed. The distribution is also shifted to the left suggesting that stance onset in leg 4 most often occurs within the first half of the leg 3 step cycle. The mean of the distribution is similar to the 4 in 3 relationship reported by Jamon and Clarac (1995) for adults, but with a much larger deviation. Temporal coordination between ipsilateral legs 2 and 3 is highly variable and does not display any preferred pattern, similar to coordination between anterior contralateral pairs.

\section{Step variables}

Components of the step cycle in each leg were analyzed to determine whether or not differences in interlimb coordination were accompanied by differences in the step variables. Each of the step variables shows a significant difference between anterior (2 and 3) and posterior legs (4 and 5). Mean periods in legs 2 and 3 are significantly shorter than leg 4 and 5 periods (Figure 2.5A). These data suggest that the anterior legs are cycling approximately $30 \%$ faster than the posterior legs. Stride lengths displayed similar results. Legs 2 and 3 have significantly shorter stride lengths compared to legs 4 and 5 (Figure 2.5B). Again, there was an approximately $30 \%$ difference between the mean stride lengths of anterior and posterior legs. 
A

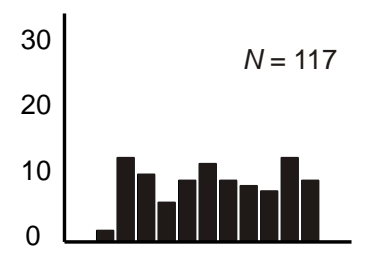

L3 in R3

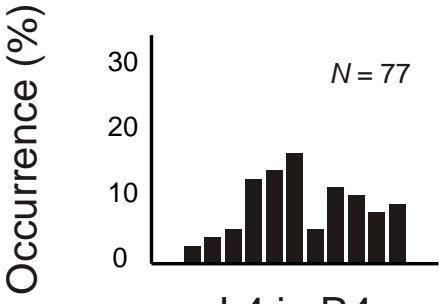

L4 in R4

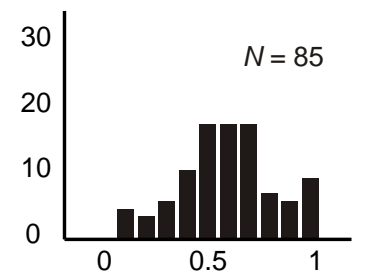

L5 in R5
B
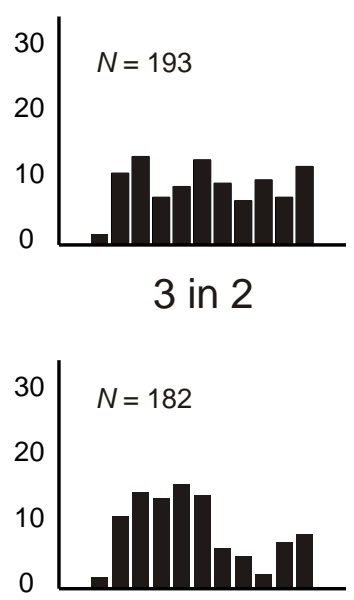

4 in 3

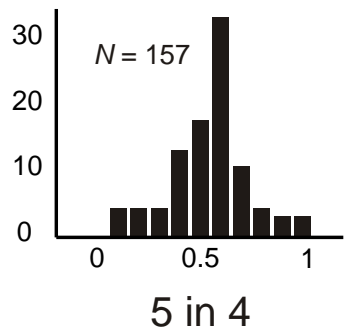

Figure 2.4. Phase relationships between neighboring legs. Histograms showing distribution of phase values in neighboring contralateral (A) and ipsilateral (B) legs observed in freely walking crayfish. A) Contralateral phase distributions show high variability in the anterior legs. The distributions in the fourth and fifth leg pairs suggest a predominantly alternating gait. B) Ipsilateral relationships show that phase values become more tightly distributed in posterior legs. The strongest relationship exists between ipsilateral adjacent legs 4 and 5. Means: 5 in $4=0.54 \pm 0.19 ; 4$ in $3=0.46 \pm 0.28 ; 3$ in $2=0.53 \pm 0.30$; 5 in $5=0.6 \pm 0.24 ; 4$ in $4=0.55 \pm 0.26 ; 3$ in $3=0.54 \pm 0.30$. 
A

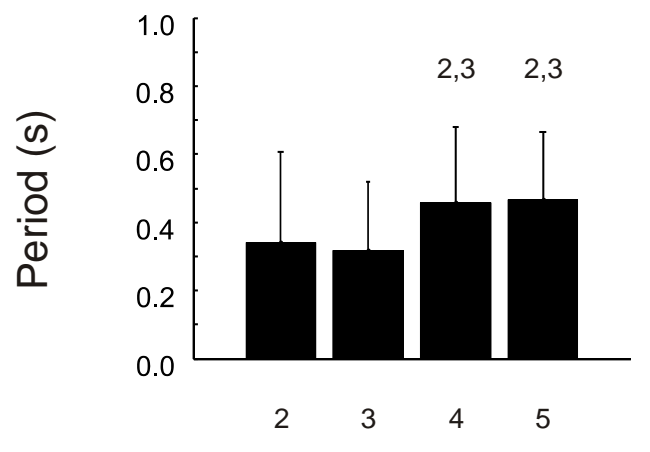

B

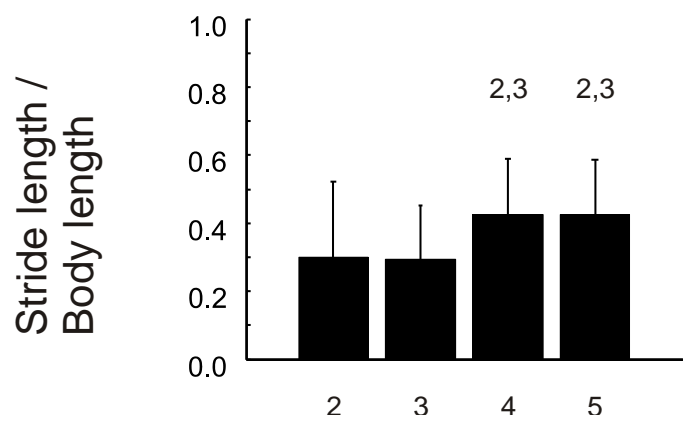

C
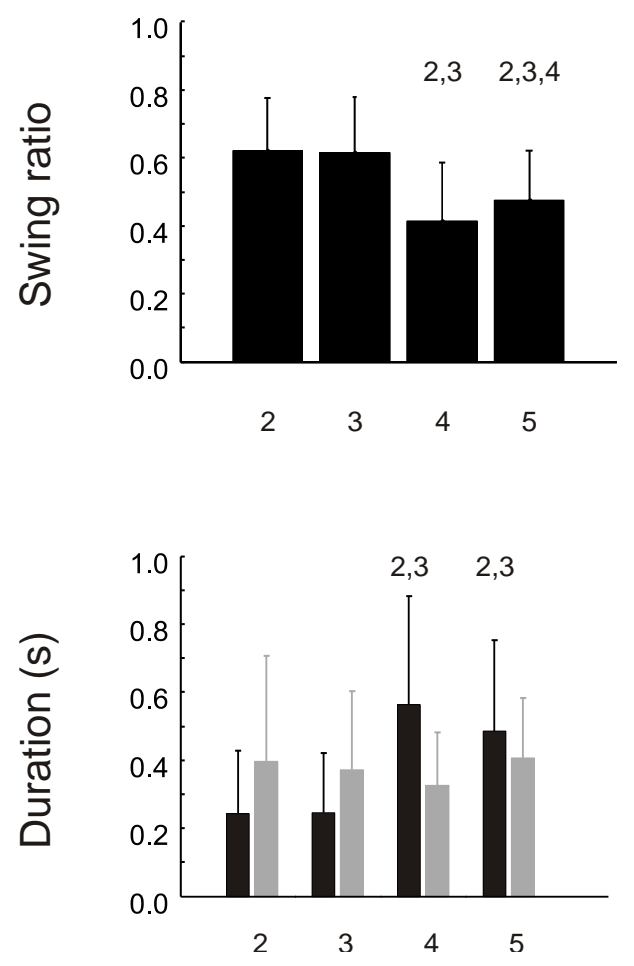

Figure 2.5. Step components of freely walking juvenile $P$. clarkii. A) Period durations are significantly longer in the posterior two pairs of legs. B) Normalized stride lengths are also larger in legs 4 and 5. C) The posterior pairs of legs remain in contact with the substrate for a longer proportion of their steps relative to anterior legs. D) Mean stance (black bars) and swing (gray bars) durations showing absolute values for each phase of the step cycle ( $x$-axis shows leg number; 2 , significant difference compared to leg $2 ; \mathbf{3}$, significant difference compared to leg $3 ; \mathbf{4}$, significant difference compared to leg 4 . One-way ANOVAs were used for statistical analysis. All differences have a $p$-value $<0.001$ ). 
Differences between stance and swing phases were observed in anterior and posterior legs. On average over $60 \%$ of the step cycle in the anterior legs is devoted to the swing phase (leg 2, 0.62 \pm 0.16 ; leg 3,0.61 \pm 0.16 ) (Figure 2.5C). Mean swing ratios of anterior legs were significantly higher than posterior legs (leg 4, $0.41 \pm 0.17$; leg 5, $0.47 \pm 0.15$ ). Legs 4 and 5 spent more of their step cycle in contact with the substrate. Mean stance durations were twice as large in the posterior legs (black bars, Figure 2.5D) compared to anterior legs. Mean swing durations, however, were more similar across all legs (gray bars, Figure 2.5D). These data show that both relative and absolute stance durations are longer in the posterior pairs of legs, while the duration of swing phase is more consistent across all legs.

Correlations were found between stance and swing durations against period (Figure 2.6). Periods in posterior legs show a stronger correlation with stance durations compared to swing. The opposite is true for the anterior legs 2 and 3 where the period is more strongly correlated with swing duration. These data suggest that although swing durations are similar across legs, they change proportionally with period duration within legs 2 and 3. In legs 4 and 5, stance durations change proportionally with period.

\section{Leg kinematics of juvenile crayfish}

Leg kinematics were analyzed next to characterize limb movements and determine joint contributions during walking. The data show almost no angular excursion in the rostro-caudal moving joints, $\mathrm{TC}+\mathrm{IM}\left(<10^{\circ}\right)$, in the anterior legs (first column, Figure 2.7). Leg 4 shows more angular displacement with a range of motion of approximately $15^{\circ}$ per animal. Leg 5 shows the largest range of motion $\left(\sim 30-40^{\circ}\right)$.

Angular excursion of the CB joints showed similar trends. The CB joint displayed very little movement in legs 2 and $3\left(<10^{\circ}\right.$ mean range of motion; second column, Figure 2.7). The leg 4 $\mathrm{CB}$ joint had a larger range of motion $\left(>10^{\circ}\right)$. The inflection of the leg $4 \mathrm{CB}$ joint during a step suggests that the meropodite begins the stance phase with the distal end dorsal to the transverse plane of the thorax. During the stance phase, the CB joint displaces the meropodite toward a more parallel orientation with the body plane. The CB joint increases its angle during swing. 
2

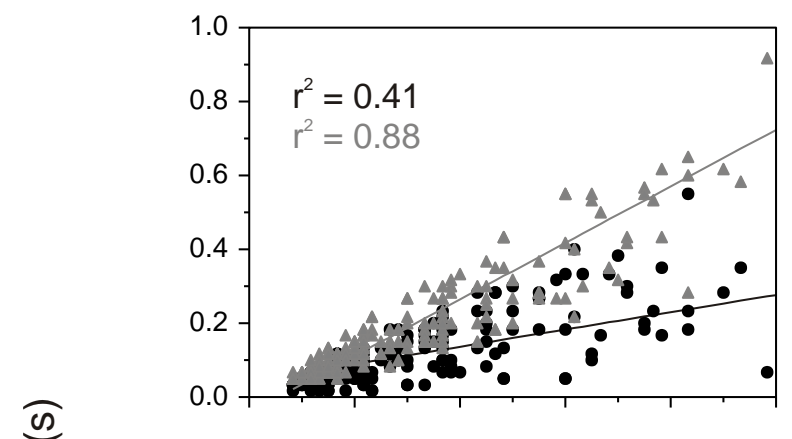

3

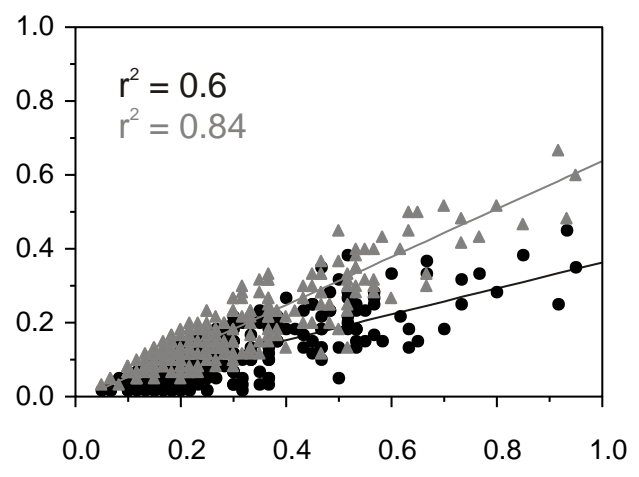

4

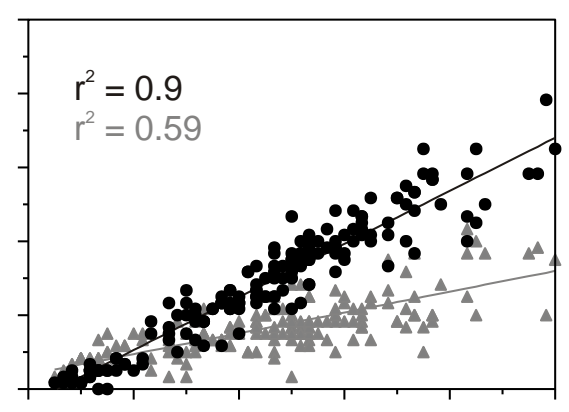

5

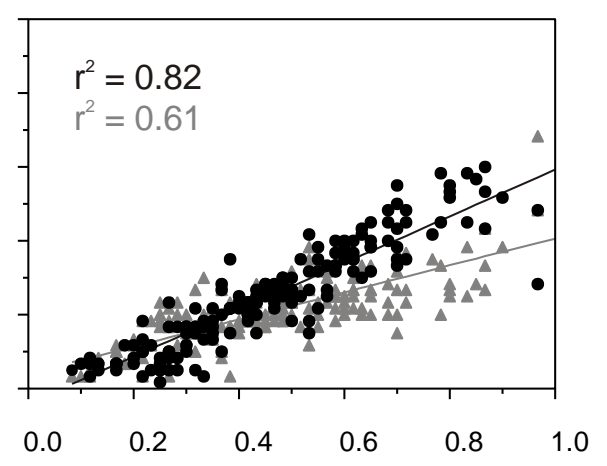

Period (s)

Figure 2.6. Linear regressions of stance (black circles) and swing (gray triangles) durations against period ( $x$-axis) durations of the anterior legs (left column) and posterior legs (right column). All $p$-values $<0.0001$. 
Therefore, the distal end of the meropodite is depressed in a downward direction relative to the body during stance and is elevated during swing. The leg 5 CB joint has a similar trajectory with greater range of motion $\left(>30^{\circ}\right)$. The plane of the meropodite depresses farther in leg 5 than leg 4. The $\mathrm{CB}$ joint produces angles $<0^{\circ}$ during stance, displacing the distal meropodite past parallel to the body plane. CB joint activity suggests that legs 4 and 5 may contribute more postural support to the body during walking than legs 2 and 3. The negative displacement of leg 5 CB joint also suggests that this leg may be crucial in providing postural support for the posterior portion of the animal.

The MC joint also showed the largest ranges of motion within each leg. The MC joint, which moves the distal portion of the leg, showed more activity in the anterior legs compared to the other joints in these legs. Leg 3 consistently displayed the smallest ranges of motion $\left(\sim 15-20^{\circ}\right)$ in all animals. The leg $2 \mathrm{MC}$ joint showed great variability between animals. One animal showed almost no MC joint activity while two other animals displayed ranges of motion near $40^{\circ}$. The leg $4 \mathrm{MC}$ joint also showed greater variability compared to its proximal joints $\left(20-40^{\circ}\right.$ range of motion). The anterior three walking legs (2-3-4) produced similar activity in the MC joint. In these legs, the MC joint flexed the distal leg during stance causing a smaller angle in the joint then extended it during swing. In contrast, the leg $5 \mathrm{MC}$ joint extended the distal portion of the leg during stance and flexed it during swing. These are typical movement patterns for the distal leg of many multi-legged animals (Ritzmann et al., 2004). The leg 5 MC joint also produced the largest ranges of motion $\left(40-50^{\circ}\right)$ for all joints in all legs.

Angular velocities were largest in those joints that displayed the largest ranges of motion. Peak velocities in these joints were achieved during the swing phase (Figure 2.8). The swing phase began with increasing velocity that peaked approximately midway through the swing before slowing just before stance. Therefore, leg 5 angular velocities were the fastest with the TC + IM excursion reaching mean peak velocities near $300^{\circ} \mathrm{s}^{-1}$. The $\mathrm{CB}$ and MC joints reached a maximum angular velocity of $400^{\circ} \mathrm{s}^{-1}$ as the leg was returned to stance. During stance phase, the leg 5 joints also showed an increase in velocity, which peaked then changed inflection towards the end of stance. However, these velocities were consistently smaller with peak mean velocities near $200^{\circ} \mathrm{s}^{-1}$ independent of the joint. Most leg joints showed fairly constant stance velocities with larger velocity changes occurring during swing. The leg $4 \mathrm{MC}$ joint was the only other joint 

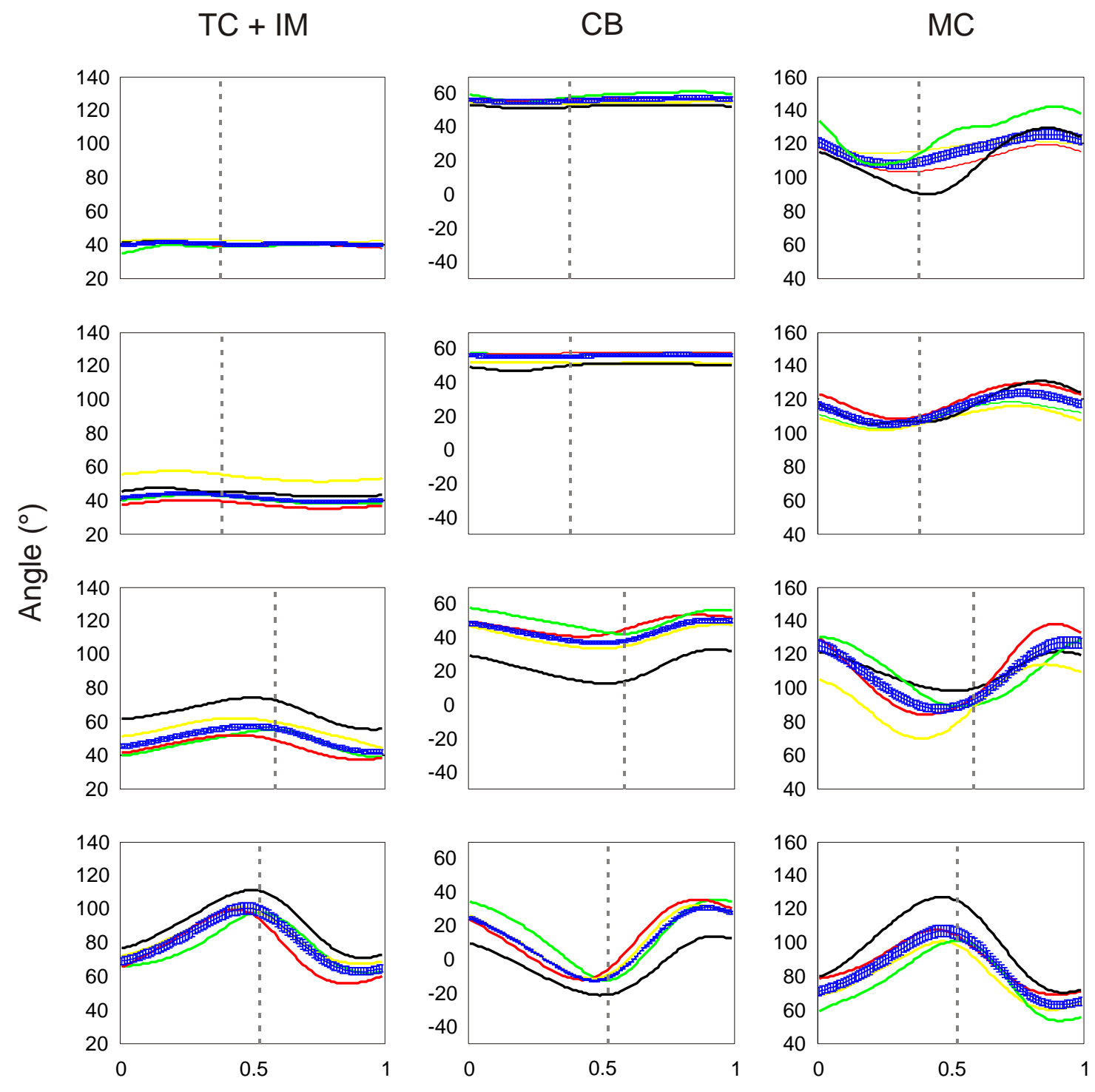

4
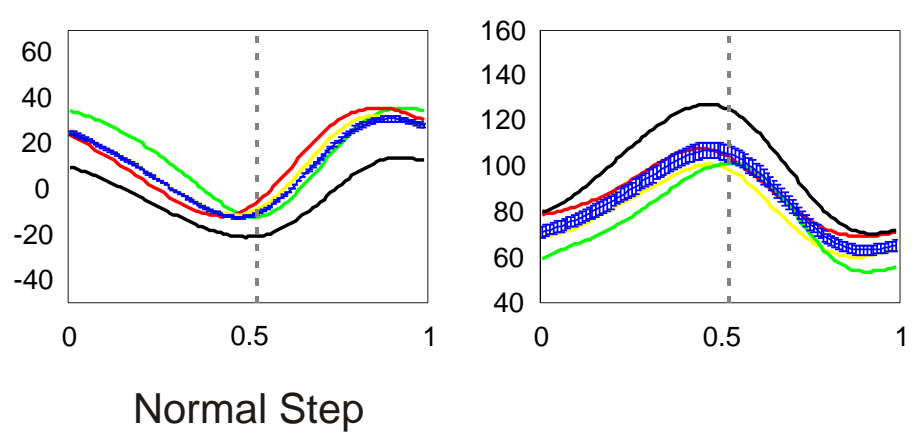

5

Figure 2.7. Kinematics of the proximal joints from legs 2-5 of juvenile $P$. clarkii during freely walking. The figure shows the mean angle ( $y$-axis) of each joint during a normalized step ( $x$-axis). Mean angles are plotted for four animals (black, red, yellow, green) as well as the mean angles for all animals (blue, mean \pm s.e.m.). Rows (top to bottom): Legs are displayed from anterior to posterior (2-5). Columns (left to right): Excursion angles (TC + IM), CB, and MC. Dashed vertical line references the mean stance/swing transition. Area to the left of the line represents mean stance duration. 
$\mathrm{TC}+\mathrm{IM}$
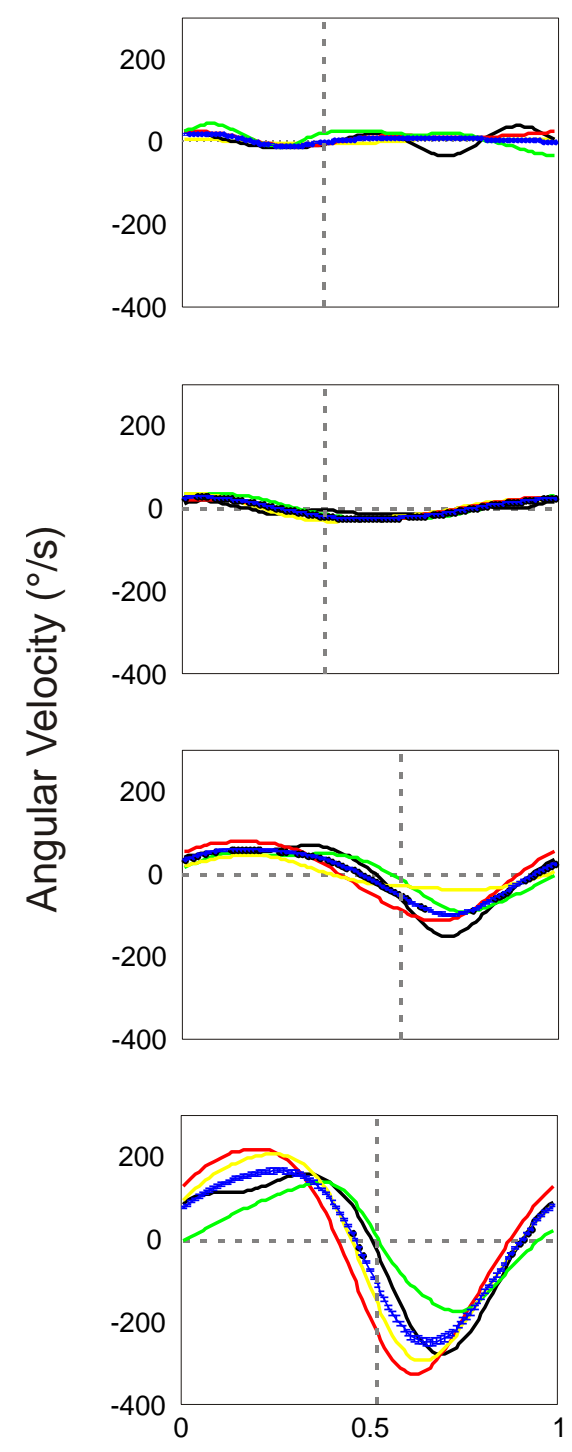

$\mathrm{CB}$
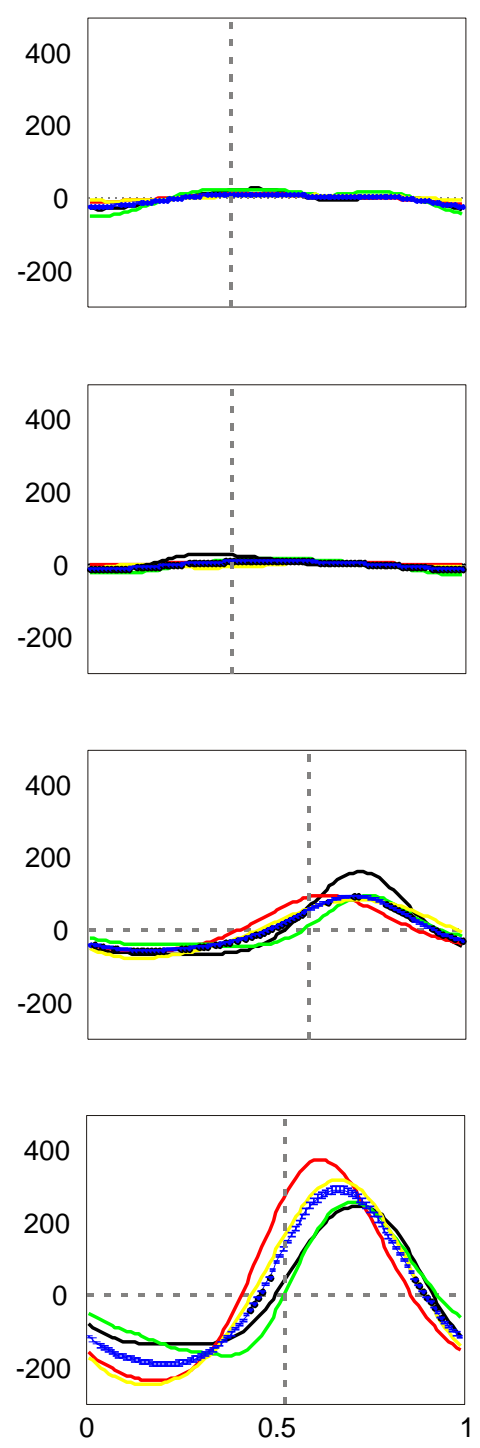

Normal Step
$\mathrm{MC}$
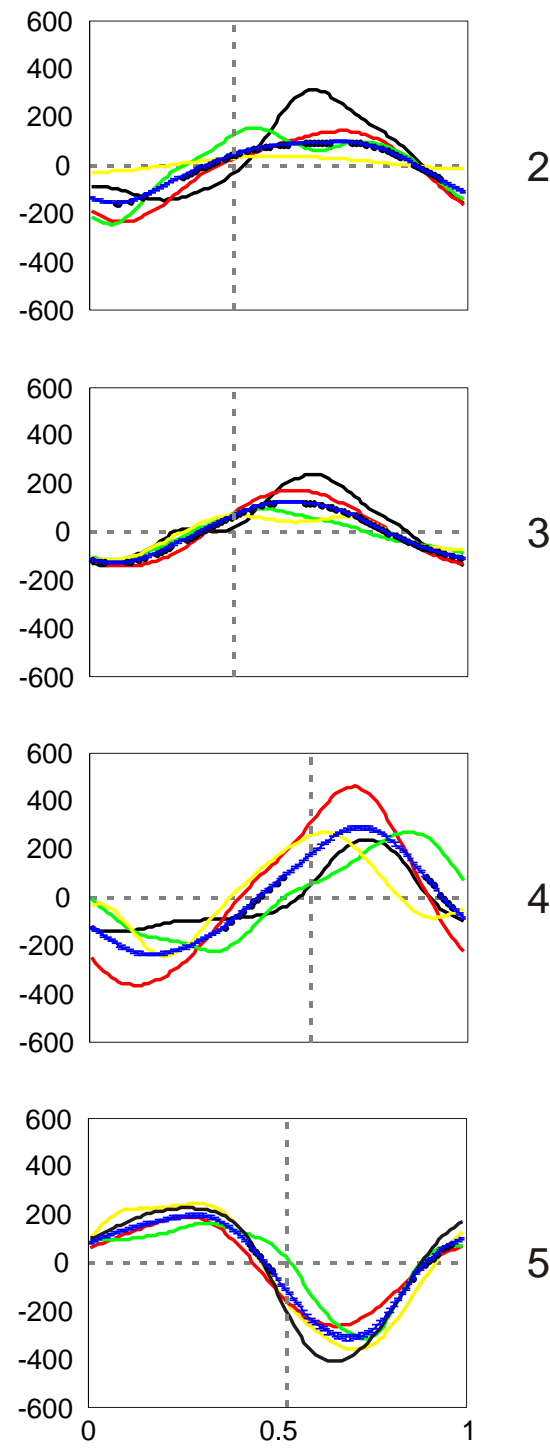

Figure 2.8. Angular velocities of the proximal joints from legs 2-5 of juvenile $P$. clarkii during freely walking. The figure shows the mean angular velocity ( $y$-axis) of each joint during a normalized step ( $x$ axis). Mean velocities are plotted for all four animals (black, red, yellow, green) as well as the mean velocities for all animals (blue, mean \pm s.e.m.). Rows (top to bottom): Legs are displayed from anterior to posterior (2-5). Columns (left to right): Excursion velocities (TC + IM), CB, and MC. Dashed vertical line references the mean stance/swing transition. Area to the left of the line represents mean stance duration. The dashed horizontal line indicates zero angular velocity. 
to reach velocities similar to those in leg 5. Velocities in the leg $4 \mathrm{MC}$ joint showed the greatest amount of variability between individuals with the exception of the animal that showed very little excursion of the leg $2 \mathrm{MC}$ joint. This may suggest multiple functions in this joint.

\section{Discussion}

The primary focus of this study was to characterize walking behavior of juvenile P. clarkii. Results obtained here can be compared with those of Jamon and Clarac $(1995,1997)$ on freely walking adult crayfish to determine how locomotor behavior changes with ontogeny. Previous work on ontogeny of pedestrian locomotion has focused mainly on mammals (Muir, 2000; rodents, Schilling, 2005; chickens, Bekoff, 1976; cats, Howland et al,. 1995; humans, Forssberg, 1984). All of these animals show generally similar body morphologies between newborns and adults but relative differences in size are not as great as those observed in crayfish. Studies on invertebrates tend to focus primarily on locomotor changes between developmental stages in holometabolous insects (Johnston and Levine, 1996). Crayfish are a model system that displays large-scale changes in body size with relatively little change to overall body morphology.

Locomotor behavior of freely walking juvenile crayfish is different than that observed in freely walking adult crayfish (Jamon and Clarac, 1995; Barnes, 1977) and lobsters (Clarac and Chasserat, 1983; Ayers and Clarac, 1978). Stepping order of the ipsilateral legs for juvenile $P$. clarkii is the same, on average, as that seen in adult Astacus (Clarac and Barnes, 1985). However, anterior ipsilateral legs in juvenile crayfish show much greater variability in limb coordination, thereby rendering gait comparisons based on stepping order incomplete. The temporal relationships between ipsilateral legs 2-4 showed more variability than in adult $P$. clarkii (Jamon and Clarac, 1995), suggesting a preferred coordination in adults that is not seen in the juveniles. Jamon and Clarac (1995) also showed two distinct coordination patterns (in phase and alternating) between the fourth legs with no preferred coordination between the fifth legs. Although juveniles displayed a few instances of in-phase coordination between both legs 4 and 5 , there was a much stronger relationship in these contralateral pairs towards alternating. 
Gait diagrams further highlight the differences in limb coordination accompanied by changes in step variables (Figure 2.3). Juveniles use predominantly alternating ipsilateral and contralateral coordination in legs 4 and 5 while legs 2 and 3 are more variable. Adults tend to display more stereotyped ipsilateral coordination between all ipsilateral neighbors (Clarac and Barnes, 1985; Jamon and Clarac, 1995) with more variable coordination in contralateral legs (Jamon and Clarac, 1995; Müller and Cruse, 1991a). Adult stepping patterns also suggest a metachronal wave of stepping activity that travels either posterior to anterior or vice versa (Clarac and Barnes, 1985). This intersegmental coordination was not observed in juvenile crayfish. This is because juvenile legs 2 and 3 cycle at a faster rate and complete at least one step before legs and 4 and 5 . Adult step periods tend to be uniform across all legs, thereby maintaining an even cycle frequency between all legs (Jamon and Clarac, 1995). Furthermore, stance durations of anterior legs in juveniles are significantly shorter than posterior legs while adults show uniformity between legs (Jamon and Clarac, 1995). Kinematic analysis of juvenile leg joints show that posterior legs have far greater ranges of motion and move at higher angular velocities than do anterior legs. Together, these findings suggest that anterior legs do not play as active a role in walking as do posterior legs or adult anterior legs. Juvenile anterior legs also show stronger correlations between swing duration and period while stance duration in posterior legs is more strongly correlated with period. When sensory feedback is reduced or eliminated, cycle durations of the leg stump or oscillatory activity in the motor neurons driving the walking muscles becomes highly correlated to the swing duration (Clarac, 1981; Chrachri and Clarac, 1990). Juveniles may be experiencing less sensory feedback in the anterior legs which results in kinematic output that more resembles an endogenous pattern generator (Sillar et al., 1987).

The basic kinematics of the four pairs of walking legs in juvenile crayfish does not differ from that previously observed in other crayfish and lobsters (Jamon and Clarac, 1997; Macmillan, 1975). The first three pairs of legs show a pulling motion during stance phase, while the last pair of walking legs displays a pushing motion. Ranges of motion and spatio-temporal coordination of the three major contributing joints (TC + IM, CB, and MC) of leg 4 also appears similar with those observed in most adult P. clarkii (Jamon and Clarac, 1997). Ayers and Clarac (1978) found co-activation of the flexor and extensor muscles controlling the $\mathrm{MC}$ joint in one of the middle legs of Palinurus. These findings suggest that during forward walking these animals are using the MC joint as a strut for support. Klärner and Barnes (1986) showed that leg 3 provided 
the most vertically-oriented force in Astacus legs suggesting postural support, while leg 5 produced the least amount of force in any direction. The small ranges of motion observed in the juvenile crayfish anterior leg joints may suggest a similar role for these legs. The large ranges of motion in leg 5 may also indicate a larger role in providing supporting and propulsive force in juveniles. However, the possibility remains that small ranges of motion in anterior legs are just a consequence of the short step periods observed in these legs. Force measurements of individual legs in three planes would allow for comparisons to distinguish whether juvenile legs have the same dynamic output as adult legs.

There are three potential causes for differences in juvenile and adult walking behavior. The first may be a difference in load distribution on the legs due to size differences of the chelae. If all other body and limb segments scale proportionally, adults with larger chelae may have a more anterior center of mass compared to juveniles. Previous studies have shown that relatively weak contralateral relationships in adult crayfish can be pushed toward an alternating coordination by loading the animals with added weight (Clarac and Barnes, 1985) or by removing the animal from water (Grote, 1981). Grote (1981) observed that loaded adult $P$. clarkii increased both their stride lengths and stance durations. This behavior was present in the posterior legs 4 and 5 . Therefore, smaller chelae may mean a posterior center of mass that result in relative loading of the posterior legs only. If the load distribution across legs is larger in the posterior legs then there may be more sensory feedback to these legs with less feedback to the anterior legs. The "unloaded" anterior legs should then behave more like a leg with reduced feedback, as they do in Figure 2.6.

Another possible explanation for different walking behavior in juveniles and adults may be the result of developmental processes in the nervous system. In another species of crayfish, Cherax destructor, Sullivan and Macmillan (2001) found that no neurogenesis occurred in the central nervous system after the animal gained the ability to walk and limbs showed complete structural development. Huxley (1880) also observed that after the last post-embryonic molt Astacus leptodactylus showed morphological changes in the fifth dactyls that resembled mature animals. This change in dactyl morphology was accompanied by the ability to perform the rhythmic motion required for walking. All of our experimental animals had fully developed limbs and showed the ability to walk and display rhythmicity across all legs. Therefore, the juveniles 
tested here were most likely equipped with walking neural circuitry similar to fully mature adults. However, even if the juvenile nervous system is fully developed, differences in synaptic plasticity may still exist within the juvenile and adult walking circuitries that come with experience and age.

A third potential cause for the observed differences in walking behavior may be the different hydrodynamic environments. If the two extreme sizes of crayfish used in our allometric comparisons were to walk at velocities of one body length s ${ }^{-1}$, the Reynolds number $(R e)$ with respect to body axis would be 89 in the juvenile and 14400 in the adult. This is a significant difference in $R e$ and would mean that the two animals are walking in separate fluid dynamic regimes (Alexander, 2003). This hypothetical situation suggests that such a difference may require a unique strategy for juvenile crayfish to deal with the more viscous environment. Increasing the vertical and horizontal spatial components in the fifth pair of legs could be the strategy adopted by the animals to overcome larger drag forces.

\section{References}

Alexander, R. M. (2003). Principles of animal locomotion. Princeton University Press, Princeton, Oxford, pp 53-67

Ayers, J. L. and Davis, W. J. (1977). Neuronal control of locomotion in the lobster. I. Motor programs for forward and backward walking. J. comp. Physiol. 115, 29-46

Ayers, J. L. and Clarac, F. (1978). Neuromuscular strategies underlying different behavioral acts in a multifunctional crustacean leg joint. J. comp. Physiol. 128, 81-94

Barnes, W. J. P. (1975). Leg coordination during walking in the crab, Uca pugnax. J. comp. Physiol. 96, 237-256

Barnes, W. J. P. (1977). Proprioceptive influences on motor output during walking in the crayfish. J. Comp. Phsiol. (Paris) 73, 543-564 
Bekoff, A. (1976). Ontogeny of leg motor output in the chick embryo: a neural analysis. Brain Res. $412,84-95$

Burrows, M. and Hoyle, G. (1973). The mechanism of rapid running in the ghost crab Ocypode ceratophthalma. J. exp. Biol. 58, 327-349

Chasserat, C. and Clarac, F. (1980). Interlimb coordinating factors during driven walking in crustacean. J. comp. Physiol. 139, 293-306

Chasserat, C. and Clarac, F. (1983). Quantitative analysis of walking in a decapods crustacean, the rock lobster Jasus lalandii. II. Spatial and temporal regulation of stepping in driven walking. J. exp. Biol. 107, 219-243

Chrachri and Clarac, F. (1990). Fictive locomotion in the fourth thoracic ganglion of the crayfish, Procambarus clarkii. J. Neurosci. 10(3), 707-719

Clarac, F. (1976). Crustacean cuticular stress detectors. In: Structure and function of proprioceptors in the invertebrates. Mill, P.J. (ed.). Chapman \& Hall, London, pp 299-321

Clarac, F. (1981). Decapod crustacean leg coordination during walking. In: Locomotion and energetic in arthropods. Herreid, C.F. and Fourtner, C.R. (eds.). Plenum Press, New York, London. pp 31-71

Clarac, F. (2002). Neurobiology of crustacean walking: from past to future. In: Crustacean Experimental Systems in Neurobiology. Ed. Konrad Wiese. Springer, Berlin, pp 119-137

Clarac, F. and Barnes, W. J. P. (1985). Peripheral influences on the coordination of the legs during walking in decapods crustaceans. In: Coordination of motor behavior. Bush, B.M.H. and Clarac, F. (eds.). Cambridge University Press, Cambridge. pp 249-269

Clarac, F. and Chasserat, C. (1983). Quantitative analysis of walking in a decapods crustacean, the rock lobster Jasus lalandii. I. Comparative study of free and driven walking. J. exp. Biol. $107,189-217$ 
Cruse, H. (1976). The function of the legs in the free walking stick insect, Carausius morosus. J. comp. Physiol. 112, 235-262

Cruse, H. and Müller, U. (1984). Short communications: A new method measuring leg position of walking crustaceans shows that motor output during return stroke depends upon load. J. exp. Biol. 110, 319-322

Delcomyn. F. (1971). The locomotion of the cockroach, Periplaneta americana. J. exp. Biol. $54,443-452$

Domenici, P., Jamon, M. and Clarac, F. (1998). Curve walking in freely moving crayfish (Procambarus clarkii). J. exp. Biol. 201, 1315-1329

Fisher, N.I. (1993). Statistical analysis of circular data. Cambridge University Press, Cambridge.

Forssberg, H. (1985). Ontogeny of human locomotor control. I. Infant stepping, supported locomotion and transition to independent locomotion. Exp. Brain Res. 57, 480-493

Full, R. J., Yamauchi, A. and Jindrich, D. L. (1995). Maximum single leg force production: cockroaches righting on photoelastic gelatin. J. exp. Biol. 198, 2441-2452

Grote, J. R. (1981). The effect of load on locomotion in crayfish. J. exp. Biol. 92, 277-288

Howland, D.R., Bregnam, B.S., and Goldberger, M.E. (1995). The development of quadrupedal locomotion in the kitten. Exp. Neurol. 135, 93-107

Jamon, M. and Clarac, F. (1995). Locomotor patterns in freely walking crayfish (Procambarus clarkii). J. exp. Biol. 198, 683-700

Jamon, M. and Clarac, F. (1997). Variability of leg kinematics in free-walking crayfish, Procambarus clarkii, and related inter-joint coordination. J. exp. Biol. 200, 1201-1213

Johnston, R.M. and Levine, R.B. (1996). Locomotory behavior in the hawkmoth Manduca sexta: kinematic and electromyographic analyses of the thoracic legs in larvae and adults. J. exp. Biol. 199, 759-774 
Klärner, D. and Barnes, W. J. P. (1986). The cuticular stress detector (CSD2) of the crayfish. II. Activity during walking and influences on leg coordination. J. exp. Biol. 122, 161-175

Laurent, G. (1986). Thoracic intersegmental interneurones in the locust with mechanoreceptive inputs from a leg. J. Comp. Physiol. A 159, 171-186

Laurent, G. and Burrows, M. (1989b). Intersegmental interneurons can control the gain of reflexes in adjacent segments of the locust by action on nonspiking local interneurons. $J$. Neurosci. 9, 3030-3039

Libersat, F., Clarac, F. and Zill, S. N. (1987a). Force-sensitive mechanoreceptors of the dactyl of the crab: single-unit responses during walking and evaluation function. J. Neurophysiol. 57, $1618-1637$

Ludwar, B. C., Göritz, M. L. and Schmidt, J. (2005). Intersegmental coordination of walking movements in stick insects. J. Neurophysiol. 93, 1255-1265

Macmillan, D. L. (1975). A physiological analysis of walking in the American lobster (Homarus americanus). Phil. Trans. R. Soc. B. 270, 1-59.

Muir, G.D. (2000). Early ontogeny of locomotor behavior: a comparison between altricial and precocial animals. Brain Res. Bull. 53, 719-726

Müller, U. and Cruse, H. (1991a). The contralateral coordination of walking legs in the crayfish Astacus leptodactylus. I. Experimental results. Biol. Cybern. 64, 429-436

Müller, U. and Cruse, H. (1991b). The contralateral coordination of walking legs in the crayfish Astacus leptodactylus. I. Model calculations. Biol. Cybern. 64, 437-446

Pond, C. M. (1975). The role of the 'walking legs' in aquatic and terrestrial locomotion of the crayfish Austropotambius pallipes (Lereboullet). J. exp. Biol. 62, 447-454

Ritzmann, R.E., Quinn, R.D., and Fischer, M.S. (2004). Convergent evolution and locomotion through complex terrain by insects, vertebrates and robots. Arthropod Struct. Dev. 33, 361-379 
Ritzmann, R. E. and Büschges, A. (2007). Adaptive motor behavior in insects. Curr Opin Neurobiol. 17, 629-636

Schilling, N. (2005). Ontogenetic development of locomotion in small mammals - a kinematic study. J. exp. Biol. 208, 4013-4034

Sillar, K. T., Clarac, F. and Bush, B. M. H. (1987). Intersegmental coordination of central neural oscillators for rhythmic movements of the walking legs in crayfish. Pasifastacus leniusculus. J. exp. Biol. 131, 245-264

Sullivan, J. and Macmillan, D. L. (2001). Embryonic and postembryonic neurogenesis in the ventral nerve cord of the freshwater crayfish Cherax destructor. J. exp. Zool. 290, 49-60

Wendler, G. (1966). The co-ordination of walking movements in arthropods. Symp Soc Exp Biol. 20, 229-249

Wilson, D. M. (1966). Insect walking. Annu Rev Entomol. 11,103-151

Vogel, S. (1994). Life in moving fluids. The physical biology of flow. Princeton University Press, Princeton, pp. 81-105 
Chapter III: Functionally similar locomotor networks produce flexible walking behavior during crayfish ontogeny 


\begin{abstract}
Juvenile and adult crayfish, Procambarus clarkii, use slightly different walking behaviors even though the two have similar body plans. It is unknown if the nervous system of a two week old juvenile is fully developed. This study investigates how the juvenile walking motor program functions when animals are induced to walk at different speeds on a treadmill. The second part of this study investigates the endogenous component of the walking nervous system, the central pattern generator (CPG). Partially amputated limbs provide less sensory feedback to the central nervous system and stump movements are more representative of the endogenous CPG. The results showed juveniles have a more variable stepping pattern at slower induced velocities without defined gait transitions, similar to adults. Also, partially amputated ipsilateral limbs function synchronously when sensory feedback is diminished. This central coordination is the same observed in adults during similar amputation experiments (Clarac and Chasserat, 1979; Clarac and Barnes, 1985) as well as in isolated nerve cord preparations (Sillar et al., 1987). These findings suggest that juvenile and adult crayfish walk with functionally similar locomotor systems and respond to walking perturbations in the same way as fully developed adults.
\end{abstract}

\title{
Introduction
}

Crayfish show changes in interlimb coordination and stepping patterns during their ontogeny (Chapter 2). It is unknown what causes these changes or how behavioral differences are controlled by the nervous system. Furthermore, it is unknown if the walking motor program is fully developed in very young juvenile crayfish (14-28 days old). In locusts, the flight motor program exists in larvae before animals have developed functional wings and the ability to fly (Stevenson and Kutsch, 1988). In the holometabolous moth, Manduca sexta, the walking motor program exists in larval and adult stages but walking kinematics are very different (Johnston and Levine, 1996). This may be a result of differences in body morphology after metamorphosis. Juvenile and adult crayfish have very similar body and limb morphologies but they show a tremendous size difference (Chapter 2). The main goal of this study is to determine whether or not the functional walking motor program is the same during crayfish ontogeny. The results 
would either incriminate or rule out nervous system development as a cause of behavioral differences in walking.

Crayfish reach juvenile stage after one or two post-embryonic stages (species-dependent) ranging from 14-28 days after hatching (Sullivan and Macmillan, 2001). Upon completion of the final post-embryonic larval molt juvenile crayfish show rhythmic walking behavior in the limbs (Sullivan and Macmillan, 2001; Huxley, 1880). In Cherax destructor, no additional neurogenesis occurred in the central nervous system (CNS) after completion of this last postembryonic molt (Sullivan and Macmillan 2001). In Astacus leptodactylus, after the last postembryonic molt the juveniles show the first sign of rhythmicity in the walking legs which occurs at the same time as a morphological change in the dactyls of the fifth legs. At this stage of development the dactyls molt from an inward curved structure, believed to be used for clinging to the mother's swimmerets, into an elongated adult morphology more suited for walking (Huxley, 1880).

Forward-walking arthropods show a large range in relative walking velocities. As in many animals (Alexander, 2003), those that have the ability to move at high speeds often show gait transitions between low and high velocities (soldier crabs, Sleinis and Silvey, 1980; cockroaches, Delcomyn, 1971, Full and Tu, 1991). However, slower walking arthropods, including Procambarus and other closely related forward walking crayfish and lobsters, tend to display only one gait that becomes more stereotyped as they increase speed (Chasserat and Clarac, 1983; Clarac and Chasserat, 1986). Most forward walking decapod crustaceans use an alternating tetrapod gait, or slight variation thereof, where two separate tetrapods defined by non-adjacent limbs move in alternation with each other (Macmillan, 1975). However, this is only an idealized description because animals can show variability in gait at different walking speeds. Freely walking juvenile crayfish show more stereotyped coordination in posterior legs 4 and 5 and more variable coordination in legs 3 and 4 (Chapter 2). If juvenile and adult crayfish have similar nervous control mechanisms over walking behavior, juveniles should show more variability in interlimb coordination at slower speeds.

A second goal of this study was to describe endogenous coordination of the walking legs by reducing feedback from the legs to the CNS and to determine if the endogenous behavior differs 
from that observed in adults. Adult crayfish and lobsters show highly stereotyped coordination in ipsilateral neighboring legs during walking (Clarac, 1982; Jamon and Clarac, 1995; Cruse and Müller, 1985). Like most other pedestrian arthropods, stepping kinematics show that a leg often contacts the substrate to free adjacent legs from stance and begin their swing phases (Hughes, 1952; Cruse and Müller, 1985; Cruse, 1990; Cruse et al., 1998). Contralateral limb coordination shows a higher degree of variability (Müller and Cruse, $1991 \mathrm{ab}$; Jamon and Clarac, 1995). In isolated nerve cord and reduced-feedback preparations (limb amputations) where sensory feedback is eliminated or reduced, the centrally generated rhythm is synchronous between ipsilateral segments (isolated nerve cord, Sillar et al., 1987; amputation experiments, Clarac and Chasserat, 1979; Clarac and Barnes, 1985) and nonexistent between contralateral segments (Sillar et al., 1987). Under normal conditions, juvenile crayfish show strong ipsilateral limb coupling in the posterior two legs only. In these same legs, contralateral coordination is more stereotyped than that observed in adults (Chapter 2; Jamon and Clarac, 1995). Selected legs were partially amputated to determine if reducing feedback to the walking CPG results in similar coordination as that seen in adults. This technique has been used before to identify coordination of the walking CPGs in other decapods crustaceans (Jasus lalandii, Clarac and Chasserat, 1979; Astacus leptodactylus, Clarac and Barnes, 1985). If juvenile and adult CPGs are functionally similar then coordination of the amputated juvenile stumps should acquire a synchronous rhythm.

\section{Materials \& Methods}

\section{Animals}

Juvenile crayfish were obtained from the Louisiana State Aquaculture Center (Baton Rouge, LA, USA) and housed in a 5 liter tub equipped with an aerating stone (described in Chapter 2). The four crayfish used for this study had the following body size dimensions: animal 1) mass = $0.0135 \mathrm{~g}$, body length $=0.806 \mathrm{~cm}$; animal 2) mass $=0.0224$, body length $=1.015 \mathrm{~cm} ;$ animal 3 ) mass $=0.0207 \mathrm{~g}$, body length $=1.014 ;$ animal 4 ) mass $=0.0274 \mathrm{~g}$, body length $=1.059 \mathrm{~cm}$. 


\section{Experimental procedure}

An underwater treadmill was constructed to observe juvenile crayfish under controlled conditions. The area available to the animals for walking was approximately $4.5 \times 3.0 \mathrm{~cm}$. Juvenile crayfish were tethered to a $0.35 \mathrm{~mm}$ stainless steel wire at the center of the thorax using superglue. This provided enough support to hold the animals within the field of view of the camera while still being elastic enough to allow for some freedom of movement. The opposite end of the wire was imbedded in a small block of Sylgard $(1.2 \times 0.5 \times 0.5 \mathrm{~cm})$ that was fastened to a World Precision Instruments micromanipulator (model KITE-R). The animals were lowered onto the treadmill and filmed from overhead at 30 frames s$^{-1}$ using one stationary camera (Canon ZR60). A total of ten animals were filmed, however, only four were chosen for analysis based on completion of walking trials at all velocities (total steps: high speed, $N=1181$; medium speed, $N=815$; low speed, $N=1063$ ) and under all amputation conditions (right four, R4; and right 3 and right 4, R3 \& R4). Velocity settings were chosen based on overall success of all animals filmed. Four juvenile crayfish were induced to walk on a treadmill at three different speeds (low speed: $0.37 \pm 0.09 \mathrm{~cm} / \mathrm{s}$; medium speed: $0.48 \pm 0.10 \mathrm{~cm} / \mathrm{s}$; high speed: $0.78 \pm 0.16 \mathrm{~cm} / \mathrm{s}$ ). Normalizing treadmill speeds to body lengths of the animals yield relative speeds of $0.35-0.5$ body lengths/s at the slowest setting and 0.74-0.98 body lengths/s at the fastest setting. The fast belt speed is similar to reported freely walking velocities (Chapter 2). Treadmill speeds higher than those analyzed most often yielded trials in which the moving belt caused the crayfish to drag their legs. At belt speeds lower than the slowest setting reported yielded excessive slipping of the legs. The treadmill was marked in $1.0 \mathrm{~cm}$ increments in order to calculate the average velocity of the belt

during each trial. Stance and swing onsets were identified and digitized based on dactyl position using Peak Motus.

All of the trials used for the amputation experiments were run at the highest velocity setting as this belt speed most resembled freely walking velocities. Each crayfish was first filmed with only the right fourth leg (R4) amputated ( $N=816$ total steps), then right third (R3) and fourth legs amputated ( $N=651$ total steps). 


\section{Treatment of data}

To analyze coordination of the stepping pattern and step variables of individual steps, two events were marked and digitized per step in each leg. Stance onset was defined as the moment of contact between the dactyl and substrate, and defined spatially as the anterior extreme position $(A E P)$. The position of the dactyl in the video frame immediately before lift-off was spatially defined as the posterior extreme position (PEP). The subsequent frame was used as the temporal marker for the onset of swing phase. Stance and swing onset times of the amputated legs were marked when the stump showed a noticeable change in direction with respect to the body. Leg stumps do not make contact with the substrate; therefore, these legs do not have a true stance phase. However, a stance designation was maintained for the stumps when these limbs showed caudal-directed remotion.

The step variables measured in these experiments were calculated in MatLab using the temporal and spatial markers for stance/swing onsets (Chapter 2). One way ANOVAs were used for statistical comparison of step variables within legs across conditions. Linear regressions were used to identify correlation between stance and swing durations relative to step period for each condition. All correlations are expressed as $r^{2}$. Differences between regressions were tested for statistical significance using ANCOVAs.

Contralateral and ipsilateral phase relationships were calculated for all legs using the timing of the stance onsets. Phase values and distributions were collected using the same methods as Chapter 2.

Gait diagrams were also constructed using previously described methods (Chapter 2).

\section{Results}

Juvenile crayfish show differences between coordination and function of the anterior legs 2 and 3 and the posterior legs 4 and 5 (Chapter 2). The effects of walking speed on relative interlimb coordination and/or stepping behavior in individual limbs is reported here. 


\section{Effects of velocity on interlimb coordination}

For the most part, interlimb coordination was similar between freely walking (Chapter 2) and treadmill-driven walking. An exception to this was coordination between legs 3 and 4. During freely walking, leg R4 stance onset usually occurred in the first half of the leg 3 step (Figure 3.1). When tethered, the R4 in R3 phase distribution showed peaks between $0.4-0.5$ phase values. Belt speed appears to have little effect on this relationship. Phase relationships R3 in R2 and R5 in R4 do show changes at different walking speeds. Both become more alternating at higher belt speeds with the R5 in R4 phase relationship showing the largest percentage of alternating coordination of all legs.

Contralateral coordination did not show large differences between walking speeds (data not shown). Contralateral coordination during tethered walking was very similar to freely walking at all speeds. Treadmill and unrestrained walking showed more occurrences of alternating coordination in leg 5 while all other contralateral leg pairs showing more variability. Contralateral relationships under these conditions show lower peak occurrences than ipsilateral distributions. Therefore, walking speed does not have a large effect on contralateral phase relationships.

\section{Leg function at different velocities}

In freely walking experiments, juvenile crayfish showed shorter periods and longer relative swing durations in the anterior legs 2 and 3 compared to posterior legs 4 and 5 (Chapter 2). During treadmill-driven walking leg 4 step periods were more similar to anterior legs 2 and 3 (Figure 3.2A). Leg 5 maintains longer periods at all speeds. Mean stance durations in legs 4 and 5 are significantly greater than mean stance durations in legs 2 and 3 at all velocities except leg 4 at low speed (Figure 3.2B). At low speeds noticeable slipping was observed in the fourth legs which could contribute to the shorter mean stance duration. Legs 3-4-5 decreased stance durations as belt speed increased (again with the exception of leg 4 at low speed). Mean swing durations increased with velocity. Mean swing durations show smaller changes than stance 
$\mathrm{R} 3$ in $\mathrm{R} 2$
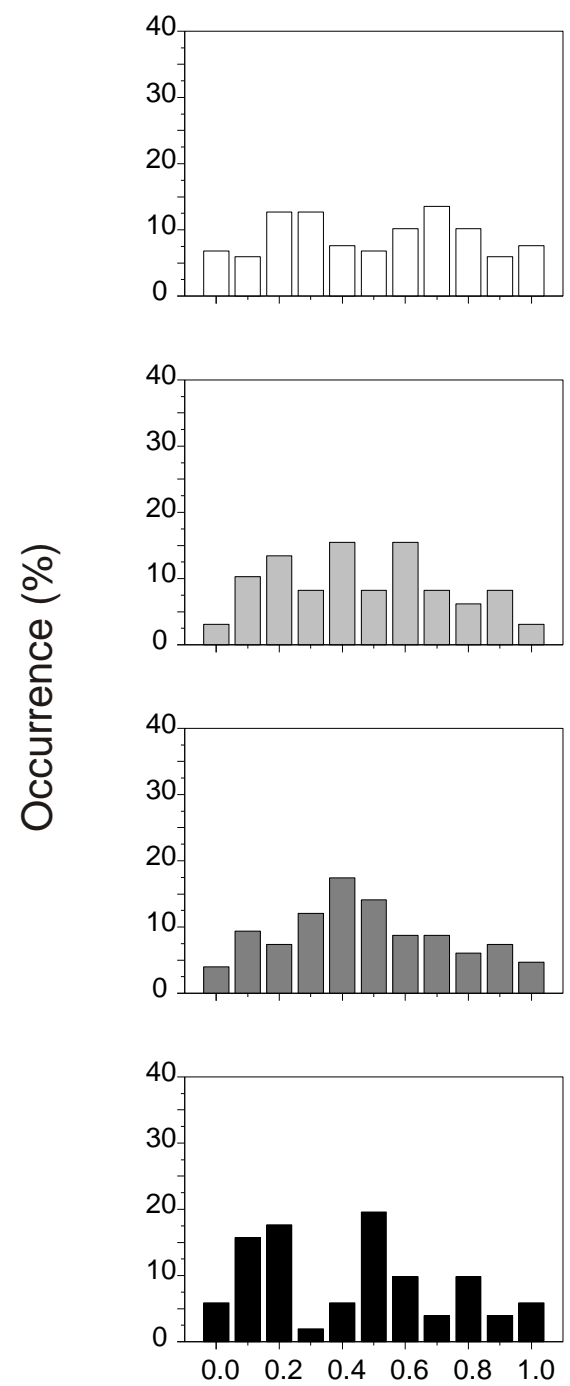

$\mathrm{R} 4$ in R3
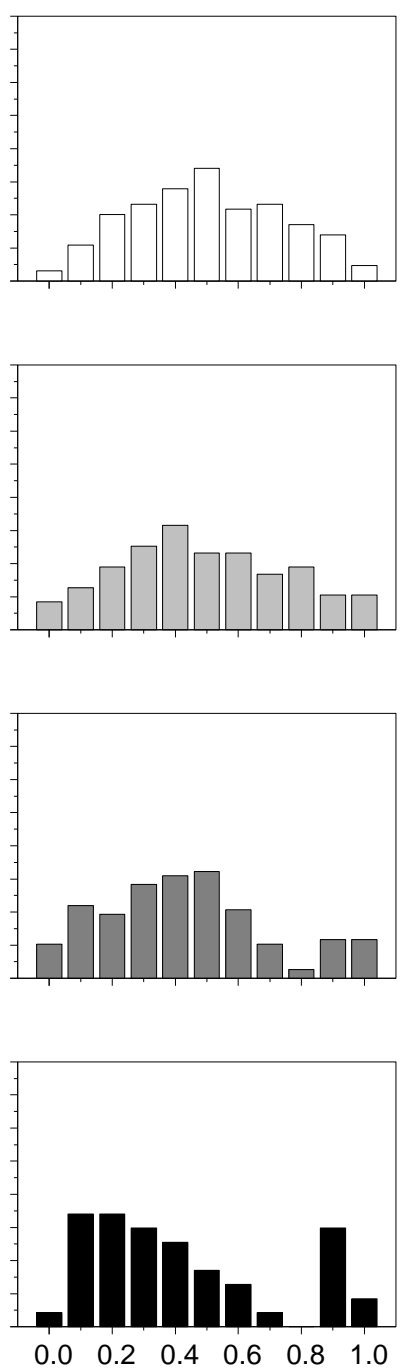

$\mathrm{R} 5$ in $\mathrm{R} 4$

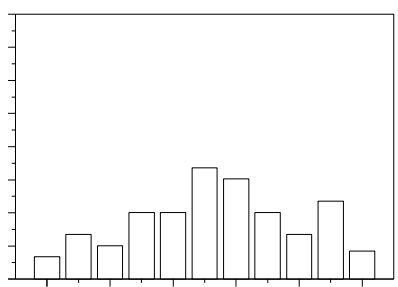

Low

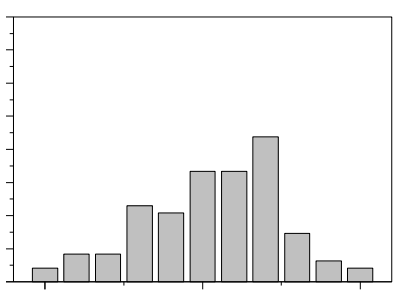

Mid

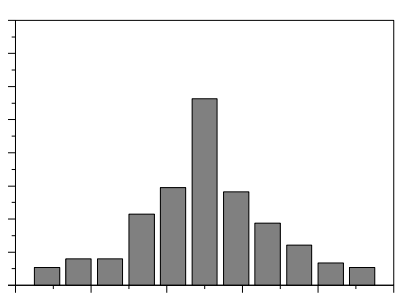

High

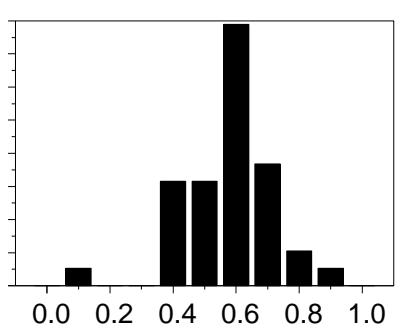

Free

Phase

Figure 3.1. Ipsilateral phase distributions observed during freely walking (black bars) and while tethered to a treadmill using varied velocity settings (high - dark gray bars; medium - light gray bars, low - open bars). A phase value of 0.5 represents an alternating coordination; whereas, a value of 0 or 1 shows inphase activity. At all speeds the coordination is more alternating with less variability in posterior limbs. However, as velocity decreases, the alternating gait becomes less pronounced in the last pairs of legs. Data for freely walking juveniles was taken from four different animals in Chapter 2. 

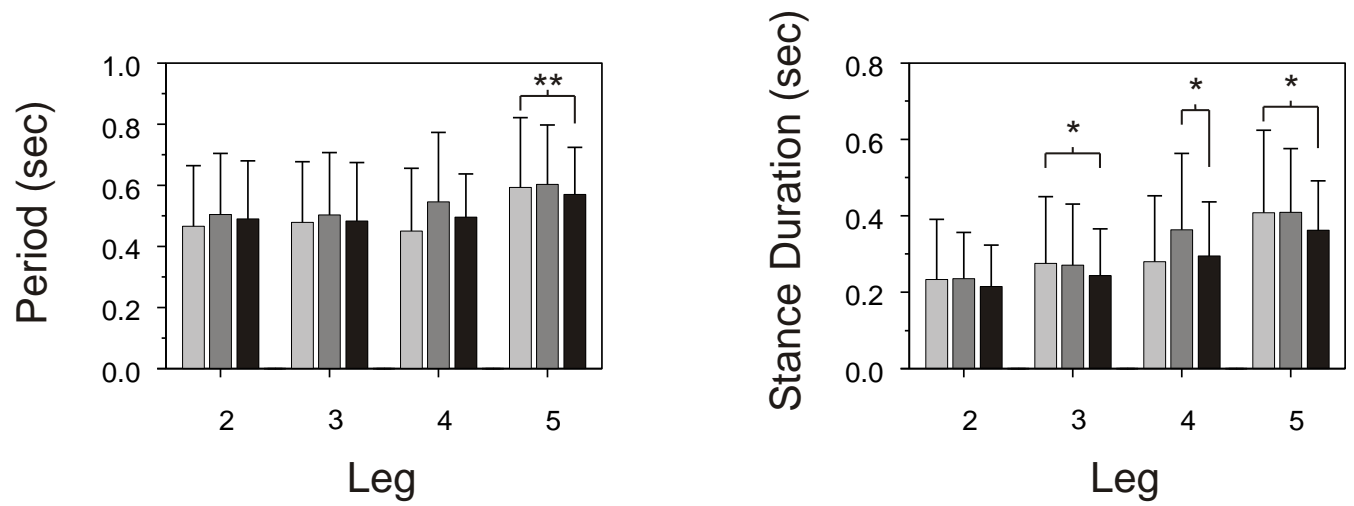

C

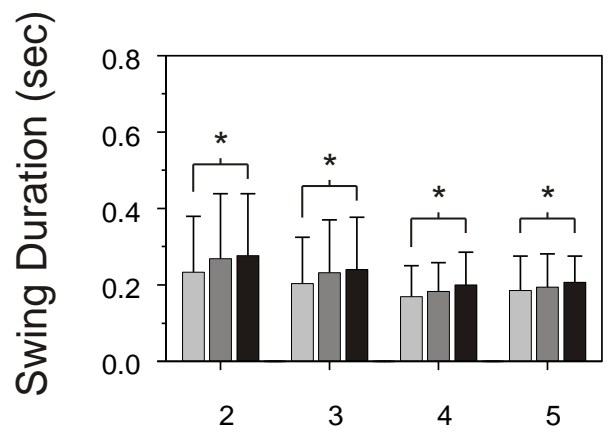

Leg

Figure 3.2. Stance and swing phases vary between anterior and posterior legs while periods show less change due to walking speed. A) **Mean periods in leg 5 are significantly larger than the mean periods of all other legs during similar induced walking speeds. The only leg to show a significant difference in period as a result of a change in belt speed was leg 4. Leg 4 showed significant differences in periods at each speed. B) Mean stance durations in legs 4 and 5 are significantly greater than mean stance durations in legs 2 and 3 at all velocities except leg 4 at low speed. *In legs 3-5 there is a significant decrease in stance duration from low to high belt speeds. Again the only exception was in leg 4 where excessive slipping may have caused a shorter mean stance duration and period. C) *There is also a significant increase in mean swing durations in all legs as belt speed increased from low to high speeds. At all speeds legs 2 and 3 were significantly different than all other legs with one exception: leg 3 vs. leg 5 at the slowest speed. (All comparisons were made using a one-way ANOVA and Tukey post-hoc test. Light gray bars indicate low belt speed: $0.37 \pm 0.09 \mathrm{~cm} / \mathrm{s}$; dark gray bars indicate medium belt speed: 0.48 $\pm 0.1 \mathrm{~cm} / \mathrm{s}$; black bars indicate high belt speed: $0.78 \pm 0.16 \mathrm{~cm} / \mathrm{s}$.) 
durations; however, the differences are still significant when comparing the slowest and fastest belt speeds (Figure 3.2C). Comparisons of legs at the same speeds show mean swing durations in legs 2 and 3 were significantly different from all other legs with the exception of the 3-5 comparison at slow speed.

Stance durations are highly correlated with periods in posterior legs at all speeds. The strongest correlations across all speeds were in the fourth and fifth legs (Figure 3.3), similar to freely walking conditions (Chapter 3). Correlations are more affected by velocity changes in anterior legs. Stance durations and periods of legs 2 and 3 became less correlated as walking speed increased. During slow walking stance duration was more highly correlated with period in legs 2 and 3. At the highest speed leg 2 and 3 swing durations showed higher correlation with period.

\section{Effects of limb amputation on ipsilateral coordination}

To determine how reducing sensory feedback from the limbs to the CNS would affect limb coordination, one then two ipsilateral legs were amputated (right leg 3 (R3), then right leg 4 (R4)). Because tethered walking at high speeds looks similar to unrestrained walking, animals were tested at this belt speed for the amputation studies.

Coordination is relatively unchanged in the left legs after amputation of leg(s) on the right side of the body (data not shown). After amputation of R4, ipsilateral phase relationships of the right legs are relatively unaffected. The R4 stump maintains a predominantly alternating coordination with R5 (top row, Figure 3.4). The R4 stump does show a slight phase shift to the first half of the R3 step cycle. There are more occurrences of an alternating coordination between legs R3 and R2 after amputation of R4. After amputation of R3, the R4 and R3 stumps were predominantly in-phase with each other (bottom row, Figure 3.5). The R5 in R4 phase relationship still showed a strong alternating coordination suggesting that both R3 and R4 stumps are beginning their "step" cycles out of phase with R5. The R3 stump also maintained a mostly alternating coordination with $\mathrm{R} 2$ but the phase distribution is more widely distributed. 

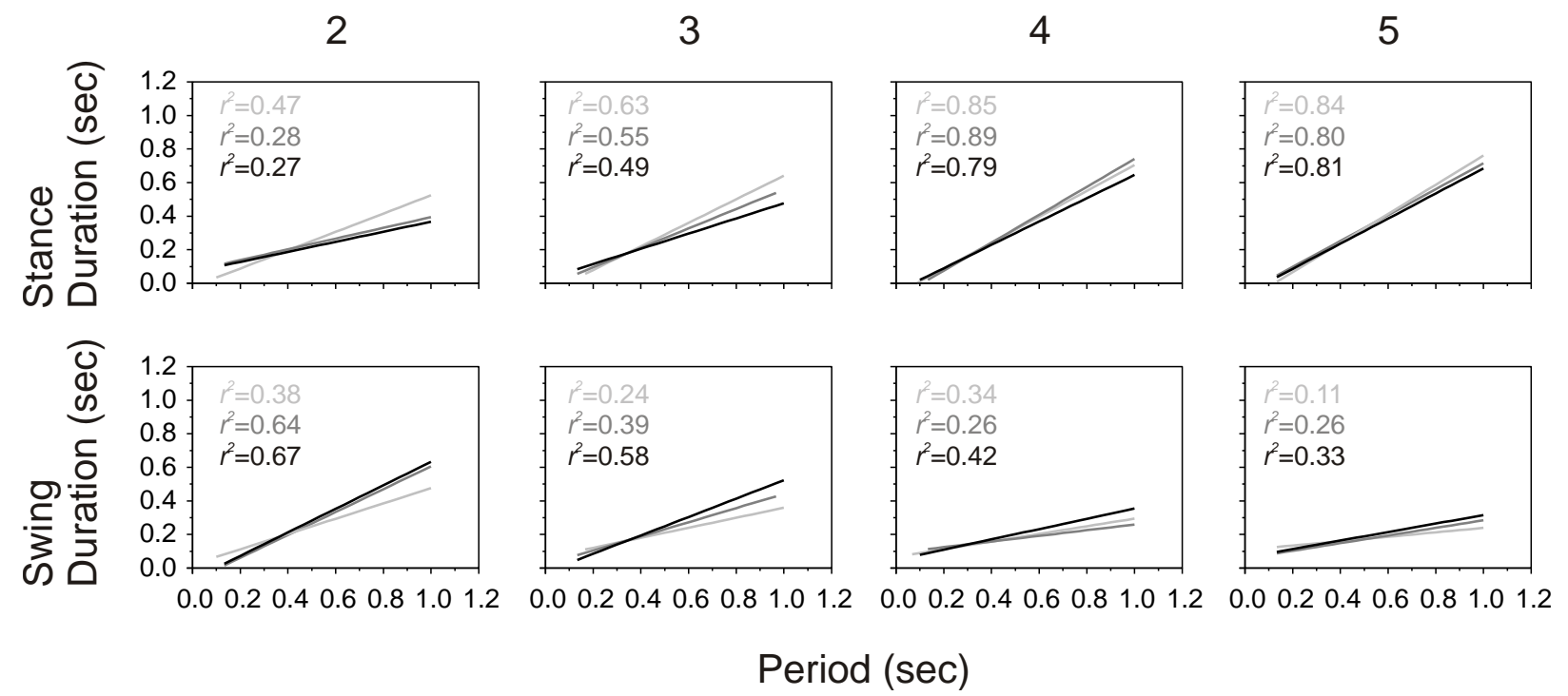

Figure 3.3. Contributions of stance and swing phase to the step period differ between legs and velocities. A) The duration of a step cycle is more closely related to the duration of the stance phase in the posterior legs 4 and 5. As velocity increases the relationship noticeably decreases in the anterior legs. (There is a significant difference between low and high speed correlations based on ANCOVAs.) B) Necessarily, the opposite is true between swing phase duration and period. There is a slightly higher correlation in the anterior legs that becomes stronger at higher belt speeds. Black line represents high speed; dark gray line represents medium speed; light gray line represents low speed. 


\section{Effects of limb amputation on contralateral coordination}

Contralateral phase relationships did not show any more or less variability between legs. Intact legs 2-4 showed variable coordination before any amputations. After amputation of R4 and R3 there was no evidence of newly acquired coordination between contralateral leg pairs (data not shown). Across all conditions the R5 in L5 phase relationship showed an alternating coordination but the frequency was more widely distributed than ipsilateral relationships between R5 and R4.

\section{Effects of limb amputation on step variables}

Step variables were affected by leg amputations. Amputation of either one or two legs resulted in a significant increase in periods of both intact and amputated legs (Figure 3.5A). Step periods in the R4 stump and the adjacent intact legs (R3 and R5) were statistically the same. After amputation of R3, both the R2 and R5 intact legs and the R3 and R4 stumps all showed similar periods. Therefore, it appears that those legs adjacent to an amputated stump(s) assume a similar stepping frequency to one another whereas intact animals showed distinctions in the fifth pair of legs.

R2 and R3 both increased stance duration after amputation of R4 (Figure 3.5B). R2 further increased stance duration after R3 was amputated. Under this condition, the R2 stance duration showed no statistical difference from R5 stance duration and was by far the largest observed in that leg across all experimental conditions. Both stumps (R3 and R4) showed a significant decrease in stance duration after amputation. Conversely, R3 and R4 stumps displayed an increase in swing duration. Intact legs did not change swing durations after amputations to nearby legs (Figure 3.5C).

Stance durations of the intact fourth leg showed a strong correlation to the step period. The same comparison after amputation of $\mathrm{R} 4$ shows very little correlation between stance duration and period (Figure 3.6). After amputation of R3 the correlation between the stump stance duration and period looks more like that of the amputated R4 with low $\mathrm{r}^{2}$. 

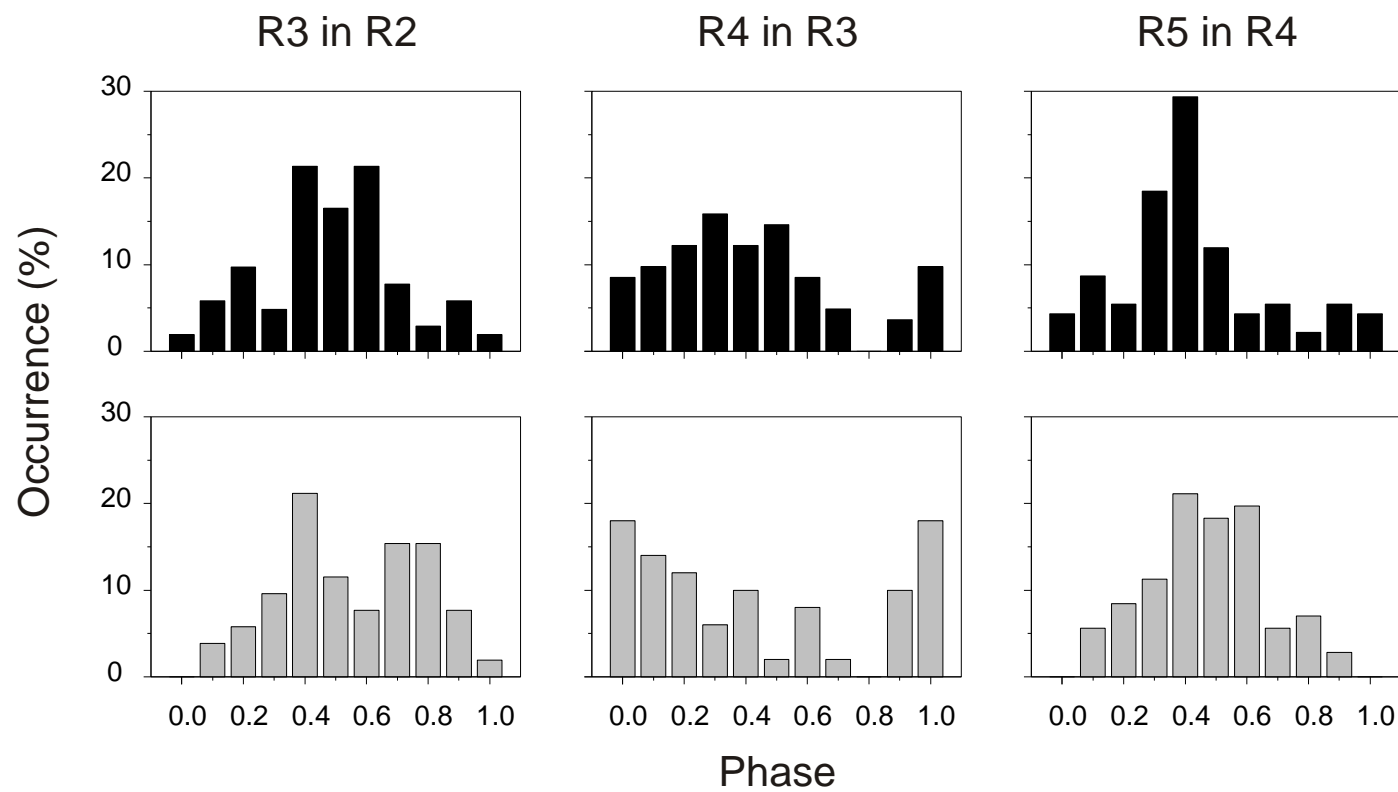

R4

R4 \& R3

Figure 3.4. Ipsilateral phase histograms describing interlimb coordination in the amputation experiments. Ipsilateral phase relationships show changes in coordination as one (R4, top row) or two legs (R3 \& R4, bottom row) are amputated. When only R4 has been amputated, there is a slightly stronger coordination between R3 and R2. The R4 stump began its power stroke predominantly within the first half of the R3 step cycle. R5 still showed an alternating coordination with R4 but with a slight phase shift toward a 0.4 phase value. When R3 was also amputated, coordination between R2 and the R3 stump became slightly more variable. The two stumps, R3 and R4, displayed a predominantly in-phase, or synchronous, coordination. R5 coordination shifted back to the middle of the R4 cycle but with a slightly wider distribution. 
A

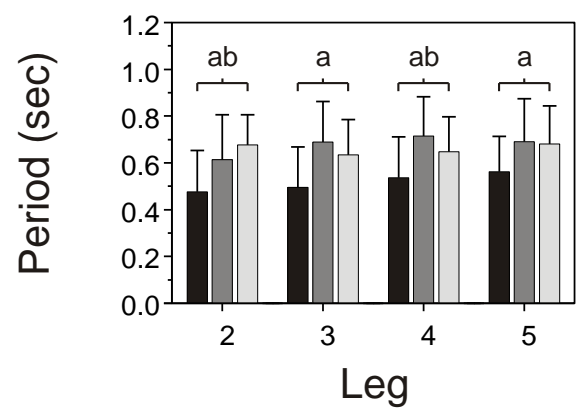

C

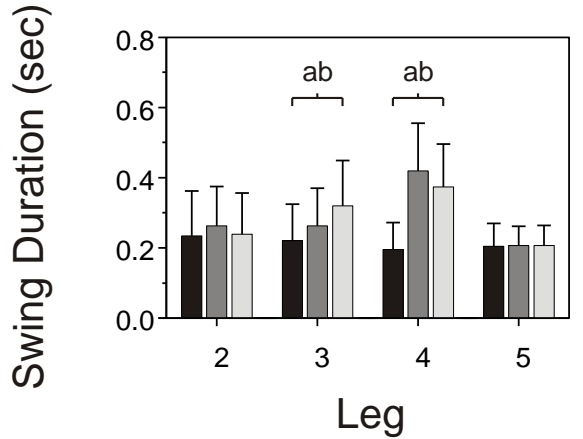

B

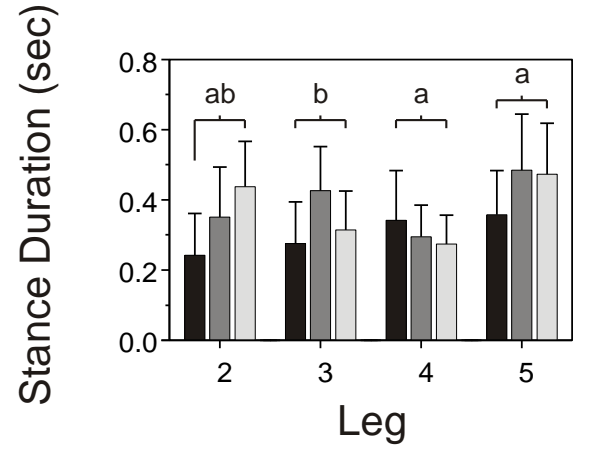

Figure 3.5. Components of the step cycle of all ipsilateral legs change after amputation of one or two legs. A) Mean periods of right legs during tethered walking at high speed (black bars) compared to periods in the same legs after amputation of R4 (dark gray bars) and R4+R3 (light gray bars). There was an increase in period across all legs after R4 was amputated (statistical significance between intact high speed vs. R4 amputation tested using one-way ANOVA and Tukey post-hoc tests and indicated by a). After amputation of R3 only the periods in legs R4 and R2 changed significantly (indicated by b). Comparing across legs showed that after amputation of R4 the periods of the intact legs R3 and R5 as well as the R4 stump became statistically similar. After amputation of R3 all leg periods were the same. B) Mean stance durations across all intact legs showed a significant increase after amputation of R4. R4 stance, or power stroke, duration decreased. After amputation of R3, R2 mean stance duration again increased while the power stroke duration in the R3 stump showed a significant decrease. Comparing between legs within each condition showed that all stance durations were statistically different after amputation of leg 4. After amputation of R3 the intact legs displayed similar stance durations that were different from the power stroke durations of the two stumps. C) The only significant changes to the mean swing duration occurred in legs 3 and 4 . When both R3 and R4 were amputated the swing durations in the intact legs R2 and R5 were the same. 


\section{Effects of limb amputation on gait}

At high speed, ipsilateral legs 4 and 5 are predominantly alternating while legs 2 and 3 are variable in their coordination with each other and the posterior legs (Figure 3.7). The posterior two legs 4 and 5 showed significantly longer stance durations while leg 5 also showed longer periods. After amputation of R4, the mean stance duration of R3 increased and coordination between R3 and R5 became predominantly alternating. It appeared that the R3 behavior assumed the role of the previously intact R4. The stump itself showed more variable coordination. Also, R4 stance duration decreased after amputation. After additional amputation of R3, mean stance duration in R2 increased significantly and showed strong alternation with R5 (right panel, Figure 3.7). Therefore, it appears that the intact limb adjacent to the amputated stump increases its role in the stepping pattern by increasing its stance duration and assuming an alternating coordination with the next adjacent intact limb. When both R3 and R4 are amputated, the coordination of the stumps becomes synchronous. The stance duration of the R3 stump decreased and resembled the stance duration of the R4 stump. Therefore, when sensory feedback is reduced in these ipsilateral adjacent limbs by amputation, the resulting coordination becomes synchronous and step parameters appear to be identical.

\section{Discussion}

Crayfish display flexible characteristics in their walking behavior during an ontogeny. Gait and step components within individual legs differ in juveniles and adults (Chapter 2). Kinematic analyses of juvenile walking suggest these animals use predominantly the posterior two pairs of legs in unrestrained conditions. Compared to adults, juveniles appear to use leg 5 in a more active role (Jamon and Clarac, 1995; Klärner and Barnes, 1986). The purpose of this study was to determine if these observed differences could be caused by developmental changes to the walking motor program. To test this hypothesis, juveniles were induced to walk at various speeds on a treadmill. Juveniles showed increasingly stereotyped behavior with an increase in walking speed. This is similar to adult Astacidea (Chasserat and Clarac, 1983; Clarac and 

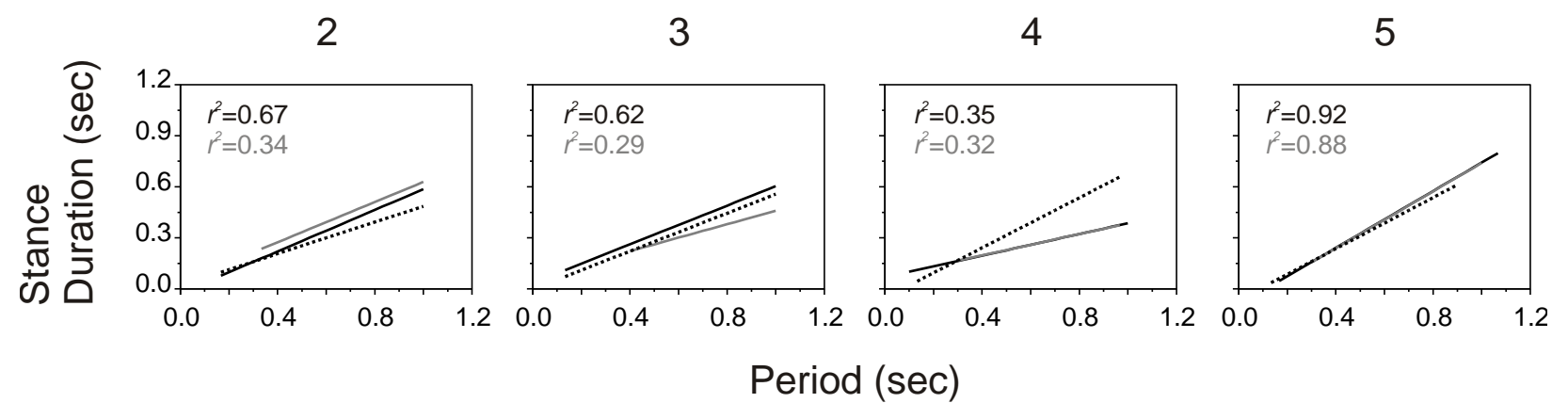

Figure 3.6. There was a statistically significant decrease in the relationship between stance duration and period in the stumps compared to the same intact legs at high speed on the treadmill. The two stumps were statistically similar. Dashed black line represents high speed intact condition; solid black line represents R4 amputated; solid gray line represents R3 \& R4 amputated. 
Chasserat, 1986) and suggests that juveniles and adult Astacidea use similar mechanisms to produce the observed behavior at different walking speeds. Legs were then amputated to reduce sensory feedback to the central nervous system to determine the endogenous properties of the leg central pattern generators. Juveniles showed a change in correlation between stance duration and period that is indicative of reduced sensory feedback to the CPG in adult crayfish and lobsters (Clarac, 1981; Chrachri and Clarac, 1990). Juveniles also displayed a new coordination in the intact legs that was the same in adult crayfish and lobsters (Clarac, 1981; Grote, 1985; Clarac and Barnes, 1985). This suggests a similar interlimb coordinating mechanism in juveniles and adults.

Faster walking speeds produce more stereotyped ipsilateral phase relationships in juvenile crayfish. Contralateral coordination is not affected by changes in walking speed. The same observations have been made in lobsters (Chasserat and Clarac, 1983). Coordination between adjacent ipsilateral legs becomes predominantly alternating as walking speed increases (Clarac and Chasserat, 1986). In both adult crayfish and lobsters, stepping frequency decreases with an increase in walking speed (Pond, 1975; Clarac and Chasserat, 1986). Leg 5 of juvenile crayfish was the only leg that showed a similar response. Jamon and Clarac (1995) have proposed that leg 4 controls the walking tempo in adult Procambarus. The results described here on juvenile crayfish may suggest that leg 5 has more of an effect on walking tempo than leg 4 . However, it is also possible that these differences are a consequence of tethering the juveniles. Freely walking juveniles show larger differences in step period between legs 3 and 4 that were not present during treadmill-walking.

Juvenile legs showed decreased stance durations and slightly increased swing durations as walking speed increases. Adult crayfish display increased swing velocities at higher walking speeds; however, swing durations in these experiments were not conclusive in their behavior (Cruse and Müller, 1984). A model of crustacean walking suggests that increasing load and/or walking speed produces shorter swing durations and longer stance durations (Cruse, 1983). Barnes (1977) showed that loading crayfish produces this response. However, results describing walking velocity effects on limb behavior of adult crayfish have yet to be reported. Lobsters also show decreased stance durations when walking faster, but swing durations in these animals are largely unaffected (Chasserat and Clarac, 1983; Clarac and Chasserat, 1986). Therefore, changes 
High Speed

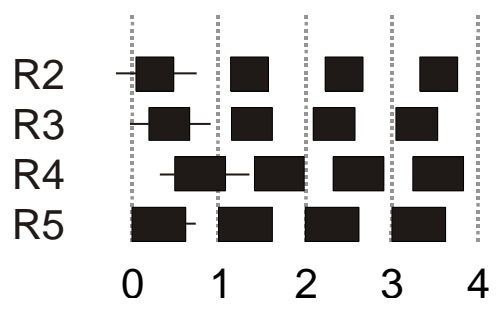

R4 Amputated

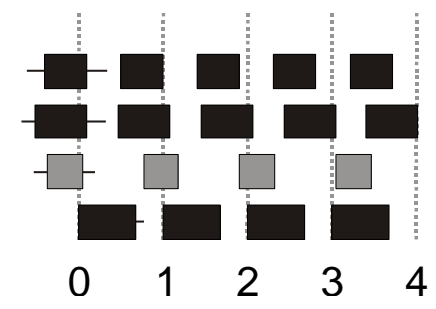

R3 \& R4 Amputated

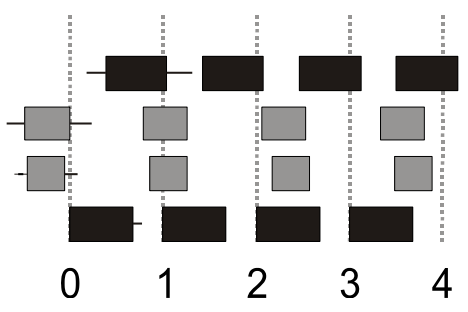

Figure 3.7. Gait diagrams of the right legs show changes in ipsilateral limb coordination before and after amputation of leg 4 and leg 3. Timing is referenced to R5 step onset. Boxes represent stance phase. A) Right legs at the high speed setting show longer stance durations in the posterior legs compared to the anterior legs. The coordination between legs R4 and R5 is predominantly alternating while coordination of the anterior legs is highly variable. B) After amputation of R4 the new coordination between the stump's power stroke (gray box) and the intact leg 5 becomes highly variable. R3 increases its stance phase and assumes a predominantly alternating coordination with leg 5. C) After amputation of R3 the two stumps assume a synchronous coordination. The intact R2 increases its stance phase and assumes an alternating coordination with leg 5 . 
in juvenile step components at various speeds are not in full agreement or contradiction with previous work. A description of the effects of walking speed on adult crayfish is required for a better comparison.

Treadmill-driven walking showed that stance durations correlated with periods strongest in the posterior legs 4 and 5. This relationship is the same for freely walking juveniles (Chapter 2). Also, velocity had less effect on this correlation in posterior legs. Anterior legs showed stronger stance duration - period correlations at slow speeds. This relationship changed at the highest walking speed where swing durations became more highly correlated with period. It has been suggested that increased sensory feedback is associated with a high correlation between stance duration and period, whereas high correlation between swing duration and period occurs when very little or no feedback is present (Clarac, 1981; Chrachri and Clarac, 1990). In juveniles anterior legs 2 and 3 may experience more feedback and contribute more to walking at slower speeds. As velocity increases anterior legs are not as active. This is similar to the behavior of fast moving cockroaches (Full and Tu, 1991), except the cockroaches undergo a drastic change in posture that totally prevents the anterior legs from being included in the stepping pattern (these cockroaches change gait). It may be simply that the effects of velocity are present in crayfish legs but the animals cannot reach speeds high enough to change posture that results in the cockroach-like behavior.

In isolated nerve cord studies on adult crayfish, coordination of oscillators in ipsilateral hemiganglia is synchronous (Sillar et al., 1987; Chrachri and Clarac, 1990). In juveniles, two adjacent amputated leg stumps oscillated in-phase with each other suggesting that with reduced sensory feedback the juvenile ipsilateral CPGs function in-phase. Synchrony was also observed in amputated ipsilateral legs of adult crayfish and lobsters (Clarac and Barnes, 1985; Clarac and Chasserat, 1979). Strong correlations between stance duration and period in juvenile posterior legs was the same as that found in posterior legs during driven-walking in lobsters (Clarac and Chasserat, 1983). When the lobsters walked backwards, the correlation became stronger in the second and third legs suggesting that the relative posterior legs are making the largest contribution to locomotion. However, when a contributing limb is amputated, the stump swing duration becomes more highly correlated with the period (Clarac, 1978; Clarac, 1981). Behavior of isolated CPGs in vitro show that the swing duration to period correlation is also predominant 
in the CNS after feedback is eliminated (Chrachri and Clarac, 1990). Juvenile leg 4 shows strong correlation between stance duration and period. After amputation of this leg, stump swing durations become more correlated with the period. This is further evidence that the properties of individual leg CPGs are the same in juveniles and adults.

Furthermore, Sillar et al. (1987) reported that no coordination between contralateral oscillators exists in the isolated nerve cords of adult crayfish. Variability in contralateral phase relationships between an intact leg and an amputated stump suggest that no coordinating mechanism exists in juveniles either. It may be that the contralateral coordination observed in the juvenile leg 5 is a result of increased sensory input from relatively larger loads on these legs. Therefore, a stereotyped contralateral coordination may be a consequence of load exchange between these posterior legs. Zill et al. (2009) has described a load mechanism in cockroach legs that may not require direct neural communication between ipsilateral legs to produce alternating coordination. There could be a similar mechanism used by crayfish that prohibits a leg from beginning its swing phase until it is sufficiently unloaded. If the same principles apply to contralateral legs, the resulting coordination may be more variable because these legs are more spatially separated than ipsilateral neighbors.

Juveniles show a change in the behavior of individual intact legs when an amputation occurs (Figure 3.8). Leg R3 increased its stance duration after R4 was amputated. R3 also showed a more alternating coordination with R5. After the additional amputation of R3, R2 displayed an alternating coordination with R5. The same responses have been observed in adult crayfish and lobsters after amputation of R4, and R3 + R4 (Clarac and Barnes, 1985; Clarac and Chasserat, 1979). Therefore, it appears that not only are the CPGs functionally similar in Astacidea, but the responses of intact legs to these perturbations are also the same. Differences in juvenile and adult walking behavior are more likely a result of morphological and environmental influences (such as relatively different load distributions based on size) rather than developmental changes to the central pattern generators. 


\section{References}

Alexander, R. M. (2003). Principles of animal locomotion. Princeton University Press, Princeton, Oxford, pp 53-67

Barnes, W.J.P. (1977). Proprioceptive influences on motor output during walking in the crayfish. J. Comp. Phsiol. (Paris) 73, 543-564

Chasserat, C. and Clarac, F. (1980). Interlimb coordinating factors during driven walking in crustacean. J. comp. Physiol. 139, 293-306

Chasserat, C. and Clarac, F. (1983). Quantitative analysis of walking in a decapods crustacean, the rock lobster Jasus lalandii. II. Spatial and temporal regulation of stepping in driven walking. J. exp. Biol. 107, 219-243

Chrachri and Clarac, F. (1990). Fictive locomotion in the fourth thoracic ganglion of the crayfish, Procambarus clarkii. J. Neurosci. 10(3), 707-719

Clarac, F. (1978). Locomotory programs in basal leg muscles after limb autotomy in the Crustacea. Brain Res. 145, 401-405.

Clarac, F. (1981). Decapod crustacean leg coordination during walking. In: Locomotion and energetic in arthropods. Herreid, C.F. and Fourtner, C.R. (eds.). Plenum Press, New York, London. pp 31-71

Clarac, F. and Barnes, W.J.P. (1985). Peripheral influences on the coordination of the legs during walking in decapods crustaceans. In: Coordination of motor behavior. Bush, B.M.H. and Clarac, F. (eds.). Cambridge University Press, Cambridge. pp 249-269

Clarac, F. and Chasserat, C. (1979). Experimental modifications of interlimb coordination during locomotion of a Crustacea. Neurosci. Lett. 12, 271-276 
Clarac, F. and Chasserat, C. (1983). Quantitative analysis of walking in a decapods crustacean, the rock lobster Jasus lalandii. I. Comparative study of free and driven walking. J. exp. Biol. $107,189-217$

Clarac, F. and Chasserat, C. (1986). Basic processes of locomotor coordination in the rock lobster. I. Statistical analysis of walking parameters. Biol. Cybern. 55, 159-170

Clarac, F. and Cruse, H. (1982). Comparison of forces developed by the leg of the rock lobster when walking free or on a treadmill. Biol. Cybern. 43, 109-114

Cruse, H. (1983). The influence of load and leg amputation upon coordination in walking Crustacea: a model calculation. Biol. Cybernetics 49, 119-125

Cruse, H. and Müller, U. (1984). Short communications: A new method measuring leg position of walking crustaceans shows that motor output during return stroke depends upon load. J. exp. Biol. 110, 319-322

Delcomyn. F. (1971). The locomotion of the cockroach, Periplaneta americana. J. exp. Biol. $54,443-452$

Full, R.J. and Tu, M.S. (1991). Mechanics of a rapid running insect: two-, four- and six-legged locomotion. J. exp. Biol. 156, 215-231

Grote, J.R. (1981). The effect of load on locomotion in crayfish. J. exp. Biol. 92, 277-288

Hughes, G. M. (1952). The coordination of insect movements. I. The walking movements of insects. J. exp. Biol. 29, 267-284

Huxley, T.H. (1880). The Crayfish: An introduction to the study of zoology. D. Appleton and Co, New York

Jamon, M. and Clarac, F. (1995). Locomotor patterns in freely walking crayfish (Procambarus clarkii). J. exp. Biol. 198, 683-700 
Johnston, R.M. and Levine, R.B. (1996). Locomotory behavior in the hawkmoth Manduca sexta: kinematic and electromyographic analyses of the thoracic legs in larvae and adults. J. exp. Biol. 199, 759-774

Klärner, D. and Barnes, W. J. P. (1986). The cuticular stress detector (CSD2) of the crayfish. II. Activity during walking and influences on leg coordination. J. exp. Biol. 122, 161-175

Macmillan, D. L. (1975). A physiological analysis of walking in the American lobster (Homarus americanus). Phil. Trans. R. Soc. B. 270, 1-59.

Müller, U. and Clarac, F. (1991a). Dactyl sensory influences on rock lobster locomotion. I. Intrasegmental and intersegmental leg reflexes during standing and walking. J. exp. Biol. 148, $89-112$

Müller, U. and Clarac, F. (1991b). Dactyl sensory influences on rock lobster locomotion. II. Role in interleg coordination. J. exp. Biol. 148, 113-128

Müller, U. and Cruse, H. (1991a). The contralateral coordination of walking legs in the crayfish Astacus leptodactylus. I. Experimental results. Biol. Cybern. 64, 429-436

Pond, C.M. (1975). The role of the 'walking legs' in aquatic and terrestrial locomotion of the crayfish Austropotambius pallipes (Lereboullet). J. exp. Biol. 62, 447-454

Sillar, K., Clarac, F., and Bush, B.M.H. (1987). Intersegmental coordination of central neural oscillators for rhythmic movements of the walking legs in crayfish, Pacifasticus leniusculus. J. exp. Biol. 131, 245-264

Sleinis, S. and Silvey, G. (1980). Locomotion in a forward walking crab. J. Com. Phsiol. 136, $301-312$

Stevenson, P.A. and Kutsch, W. (1988). Demonstration of functional connectivity of the flight motor system in all stages of the locust. J. comp. Phys. A. 162, 247-259

Sullivan, J. and Macmillan, D. (2001). Embryonic and postembryonic neurogenesis in the ventral nerve cord of the freshwater crayfish Cherax destructor. J. exp. Zool. 290, 49-60 
Zill, S.N., Keller, B.R., Duke, E.R. (2009). Sensory signals of unloading in one leg follow stance onset in another leg: Transfer of load and emergent coordination in cockroach walking. $J$. Neurophsiol. 101, 2297-2304 
Chapter IV: Kinematic analysis of crayfish walking suggests a mechanism for adapting to differential loads 


\begin{abstract}
Juvenile and adult crayfish body morphologies are proportional in size with exception of the chelae. Adult crayfish may have chelae twice as long as juveniles relative to overall body size. Longer, more massive chelae mean a more anterior center of mass. The effects of different locations of centers of mass may affect how load is distributed across the legs during walking. In this study kinematics are investigated in juveniles and adults with two different center of mass locations. Both sized animals show changes in limb behavior in the anterior and posterior legs depending on proximity to center of mass. With a more rostral location, animals increased stance durations in anterior legs and decreased stance durations in posterior legs. Juveniles and adults also use the chelae in the stepping pattern when the center of mass was more rostral. When the center of mass is caudal, the posterior legs increase stance duration and anterior legs show a decrease. Posterior legs also display increased alternating coordination with neighboring legs when the center of mass is nearer. Furthermore, the posterior leg 5 shows large changes in joint kinematics depending upon center of mass location. These findings suggest that juvenile and adult crayfish adapt similarly to changes in load distribution. It appears that individual legs can change their behavior based on the amount of relative load on the leg.
\end{abstract}

\title{
Introduction
}

Juvenile and adult crayfish, Procambarus clarkii, use different gaits during walking (Chapters 23; Jamon and Clarac, 1995). The differences are probably not due to developmental changes in the central nervous system as the endogenous activity of the limb movement is the same in both juveniles and adults (Chapter 2; Clarac and Barnes, 1985; Chrachri and Clarac, 1990). P. clarkii remain structurally similar throughout their ontogeny with the exception of the chelae, or first pair of thoracic appendages (Chapter 2). Chelae length is approximately half a body length in juveniles, while the adult claws can grow up to one body length (rostrum to caudal edge of tail fan, Chapter 2). This increase in relative length is accompanied by an increase in relative mass. Chelae mass represents $2 \%$ of the total juvenile mass, but $20 \%$ of the total mass of adults (Chapter 2). Larger chelae should result in a more anterior center of mass if all other body parts scale proportionally. To test whether this affects locomotion, adult crayfish were observed 
during unrestrained walking before and after amputation of the chelae. Juvenile crayfish were loaded to produce a center of mass location more similar to adults.

Although crayfish are decapods, they typically use only the eight posterior legs for locomotion. Adult crayfish show similar step cycle frequencies and stance and swing durations in all walking legs (Jamon and Clarac, 1995). In essence, adult crayfish are eight-legged walkers. Juvenile crayfish display differences in these step components between anterior (legs 2 and 3) and posterior (legs 4 and 5) limbs. The juveniles act more like four-legged walkers that occasionally use the anterior legs in a supporting role (Chapter 2). That is, juvenile posterior legs most often display an alternating ipsilateral and contralateral coordination and longer relative stance durations (Chapter 2-3). Increasing load on adults has the same effect on limb coordination and stepping behavior in all of the legs (Barnes, 1977; Clarac and Barnes, 1985; Grote, 1981). It may be possible that juveniles experience more load on the posterior legs due to a more caudal center of mass. Therefore, the effects of increased load may be localized to posterior limbs.

It is unknown how crayfish coordinate limb movements to produce stepping patterns. Endogenous activity of central oscillators suggests synchronous coordination in ipsilateral legs (Sillar et al,. 1987). This is contrary to the alternating coordination between neighboring legs when sensory feedback is present (Hughes, 1952; Müller and Cruse, 1984). Interlimb coordinating mechanisms have been suggested based on previous kinematic analyses (Cruse, 1990). These mechanisms assume neural connectivity between all legs. The proposed connectedness between legs is based largely on experiments that focused on whole-animal changes in load (Barnes, 1977; Grote, 1981; Cruse and Müller, 1984). Crayfish and stick insects can entrain leg oscillators through sensory feedback from muscle receptor organs (Sillar et al., 1987; Borgmann et al., 2007). This further suggests neural connectedness between legs; however, the output remains synchronous in adjacent ipsilateral oscillators. If juvenile and adult crayfish have different center of mass locations, this would mean that the nervous system has to account for relative differences in load distribution across legs. Adults and juveniles appear to have functionally similar nervous systems (Chapter 3). Therefore, a functionally similar nervous system has to account for dissimilar amounts of feedback based on relative load distributions. If individual legs can adapt to shifts in center of mass, then the effects of increasing feedback to the system may be isolated to individual feedback loops within the legs. 


\section{Methods}

\section{Experimental design}

All experiments were performed on crayfish, Procambarus clarkii, obtained from the Louisiana State Aquaculture Center (Baton Rouge, LA, USA). Three adult female P. clarkii were filmed in a 20 gallon aquarium ( 24 in $\times 12$ in $x 16$ in) at room temperature $\left(\sim 23^{\circ} \mathrm{C}\right)$. The size of the three crayfish follows: adult (A1) body length (rostrum to caudal edge of the tail fan) $=9.0 \mathrm{~cm}$, mass $=$ $53.5 \mathrm{~g}$; adult $(A 2)$ body length $=8.7 \mathrm{~cm}$, mass $=37.1 \mathrm{~g}$; adult $(A 3)$ body length $=8.3 \mathrm{~cm}$, mass $=$ $35.9 \mathrm{~g}$. Animals walked on a substrate made from a sand and Poly-Grip ${ }^{\mathrm{TM}}$ mixture that was allowed to dry and set a week before the experiment. Four juvenile P. clarkii (14-21 days old; sex unknown) were filmed in a smaller aquarium $(7.8 \mathrm{~cm} \times 2.2 \mathrm{~cm} \times 2.2 \mathrm{~cm})$ constructed of microscope slides with a Sylgard ${ }^{\mathrm{TM}}$ substrate (same methods as Chapter 2; Full et al., 1995). Juvenile size dimensions were: juvenile $(\mathrm{J} 1)$ body length $=0.81 \mathrm{~cm}$, mass $=0.026 \mathrm{~g}$; juvenile $(\mathrm{J} 2)$ body length $=0.93 \mathrm{~cm}$, mass $=0.024 \mathrm{~g} ;$ juvenile $(\mathrm{J} 3)$ body length $=0.93 \mathrm{~cm}$, mass $=0.026 \mathrm{~g}$; juvenile $(\mathrm{J} 4)$ body length $=0.97 \mathrm{~cm}$, mass $=0.028 \mathrm{~g}$.

The study consisted of four experimental conditions. Adult crayfish with larger chelae have a more rostral center of mass location. Chelae were amputated distal the plane of autotomy. This

resulted in a caudal shift in center of mass. Therefore, clawless adults have a more similar center of mass location to juveniles. Juveniles have more caudal centers of mass under normal conditions. Juveniles were loaded by gluing a small piece of wire (measuring $20 \%$ of the dry body mass) to the dorsal side of the thorax that extended over the rostrum. Loaded juveniles have more rostral centers of mass similar to adults. The effects of amputating the chelae and loading juveniles on center of mass location can be seen in Figure 4.3.

\section{Filming procedure}

Animals under all experimental conditions were allowed to walk freely while being filmed for one hour intervals. Adult crayfish were filmed from three camera angles (60 frames/sec; Canon 
GL-1; one directly overhead, two at $\sim 45^{\circ}$ angles to the first camera as well as $\sim 45^{\circ}$ from horizontal). The entire substrate was in the field of view of all three cameras during adult trials. Resolution was $780 \times 420$ pixels. Juvenile crayfish were filmed with two cameras overhead at $\sim 45^{\circ}$ of the long axis of the aquarium in either direction ( $60 \mathrm{frames} / \mathrm{sec}$; Canon model ZR60). Resolution during juvenile trials was also $780 \mathrm{x} 420$, the same as previous experiments (Chapter 2). The movies were viewed and clips were used as successful trials if the animals completed at least two steps in a straight line that did not consist of a start or a stop. A straight line qualified as no deviation in body axis trajectory greater than $15^{\circ}$. Four trials were collected for each animal under each condition.

\section{Marking and analyzing data points}

Adults were marked using Wite-Out ${ }^{\circledR}$ at 25 different points on the body and legs. The thorax was marked at the rostrum, the caudal edge of the thorax at the dorsal midline, and at the caudal end of two rostral ridges posterior and medial to the eye sockets. The abdomen was marked at the caudal edge of the tail fan along the dorsal midline. The right walking legs (2-5) were each marked on the dorsal side of the distal end of the leg segments: ischiopodite, meropodite, carpopodite, and propodite. The chelae (leg 1) were marked on the dorsal side of the distal end of the meropodite and the distal tip of the fixed portion of the dactylopodite. Juveniles were marked at the rostrum, the intersection of the midline (defined by the intestine) and the thorax, and the telson (visible by the illuminated telson muscles, Chapter 2). When loaded, the ends of the wire were also marked and digitized.

Trials were imported into 3D motion analysis software (Vicon Motus 7.0.1) and body markers were digitized for kinematic analysis. Vicon Motus calculated the center of mass for each animal. Segmental centers of mass were measured in the adult thoraces, abdomen, and chelae using the reference board method (for further descriptions see Vídal-Gadéa et al., 2008). Segmental center of mass positions were entered into Vicon Motus, which used the segmental method to calculate the overall center of mass. Due to the small mass of juvenile crayfish, it was not possible to find precise segmental center of mass locations for the thoraces, abdomen, and chelae. Instead relative segmental center of mass locations for the adults were applied to the 
juveniles substituting the percent total body mass of each juvenile body segment. This was determined to be sufficient based on the proportionality observed in body scaling relationships across crayfish sizes (Chapter 2). Vicon Motus calculated the final center of mass location based on these segmental centers of mass. When loaded, the strand of wire on the juveniles was included in the calculations.

Leg insertion points to the thorax were identified in adults to obtain accurate joint angle measurements for the proximal thoraco-coxopodite (TC) and coxo-basipodite (CB) joints. Thoraces were secured to a board dorsal-side down and the TC hinge point was measured in the $x, y$, and $z$ planes using the rostrum as the point of origin. These distances were referenced to a plane defined by the rostrum and caudal end of the rostral ridges. Each measurement was expressed as a vector in Vicon Motus which calculated the location of the insertion points for all ten of the legs relative to the thorax markers.

Spatial and temporal footfall markers were identified for all ten legs. Stance onset times were identified as the contact time of the dactyl with the substrate. The spatial coordinates for this point were classified as the anterior extreme position $(A E P)$ for that leg. Posterior extreme positions $(P E P)$ were identified as the last video frame in which the dactyl was in contact with the substrate before onset of the swing phase. The subsequent frame served as the temporal marker for swing onset.

Adult joint angles were calculated in Vicon Motus using selected spatial markers. Four joints were measured and analyzed in each walking leg (2-5) on the right side of the animal: TC, CB, ischio-meropodite (IM), and mero-carpopodite (MC) joints. TC joints were defined as the angle between the insertion points to ischiopodite segment relative to the body axis (rostrum to caudal edge of the thorax). CB joints were defined as an anatomical angle between three points: left insertion, right insertion and meropodite in the $x y$ plane. IM angles were calculated by subtracting the TC angle from a second angle defined between the insertion points to meropodite relative to the longitudinal body axis. Both the TC and IM articulate in the rostro-caudal plane relative to the body. MC angles were measured as the angle between the three points on the ischiopodite, meropodite, and carpopodite. 


\section{Treatment of data}

Walking velocities were calculated as the distance the rostrum traveled in the plane of the substrate between consecutive video frames. The mean velocity was found for all trials of each animal under both experimental conditions.

Step variables were measured using the temporal and spatial event markers similar to previous work on crayfish locomotion (Chapters 2-3). Step variables were compared in each leg before and after moving the center of mass. One-way ANOVAs were used to compare caudal and rostral conditions in each leg.

Phase relationships describe the relative temporal coordination between two legs (Chapters 2-3). Phase values were calculated as the onset of stance in leg $A$ relative to the period of the reference leg $B$, or: $\Phi_{\mathrm{A} \text { in } \mathrm{B}}=\left(\right.$ onset stance $_{A}-$ onset $\left._{\text {stance }}\right) / P_{\mathrm{B}}$.

Swing profiles were created and defined as the percent swing shared by two legs. Using MatLab (R2006a), all leg swing times were referenced to the swing times in the left fourth (L4) leg. Shared swing times were calculated as a percentage of the total L4 swing and graphed as a percentage ranging from 0 to $40 \%$ L4 swing.

Adult joint angles and angular velocities were filtered in Vicon Motus using the Butterworth method with a cutoff frequency of $2 \mathrm{~Hz}$. Data sets were sorted into individual steps and normalized to corresponding step periods (same as Chapter 2). Angles in the normalized steps were averaged for each of the two load conditions and presented as the mean \pm s.e.m.

\section{Results}

\section{Walking velocities}

Juvenile crayfish walked relatively faster than adults (Figure 4.1). The range of mean velocities for adults was approximately 1 to $3.6 \mathrm{~cm} / \mathrm{s}$, whereas the juveniles walked with mean velocities ranging from 0.5 to $1.3 \mathrm{~cm} / \mathrm{s}$. Normalizing walking velocity to body lengths per second (bl/s) showed the range of mean velocities was $0.1-0.4 \mathrm{bl} / \mathrm{s}$ for adults and $0.6-1.5 \mathrm{bl} / \mathrm{s}$ in juveniles. 

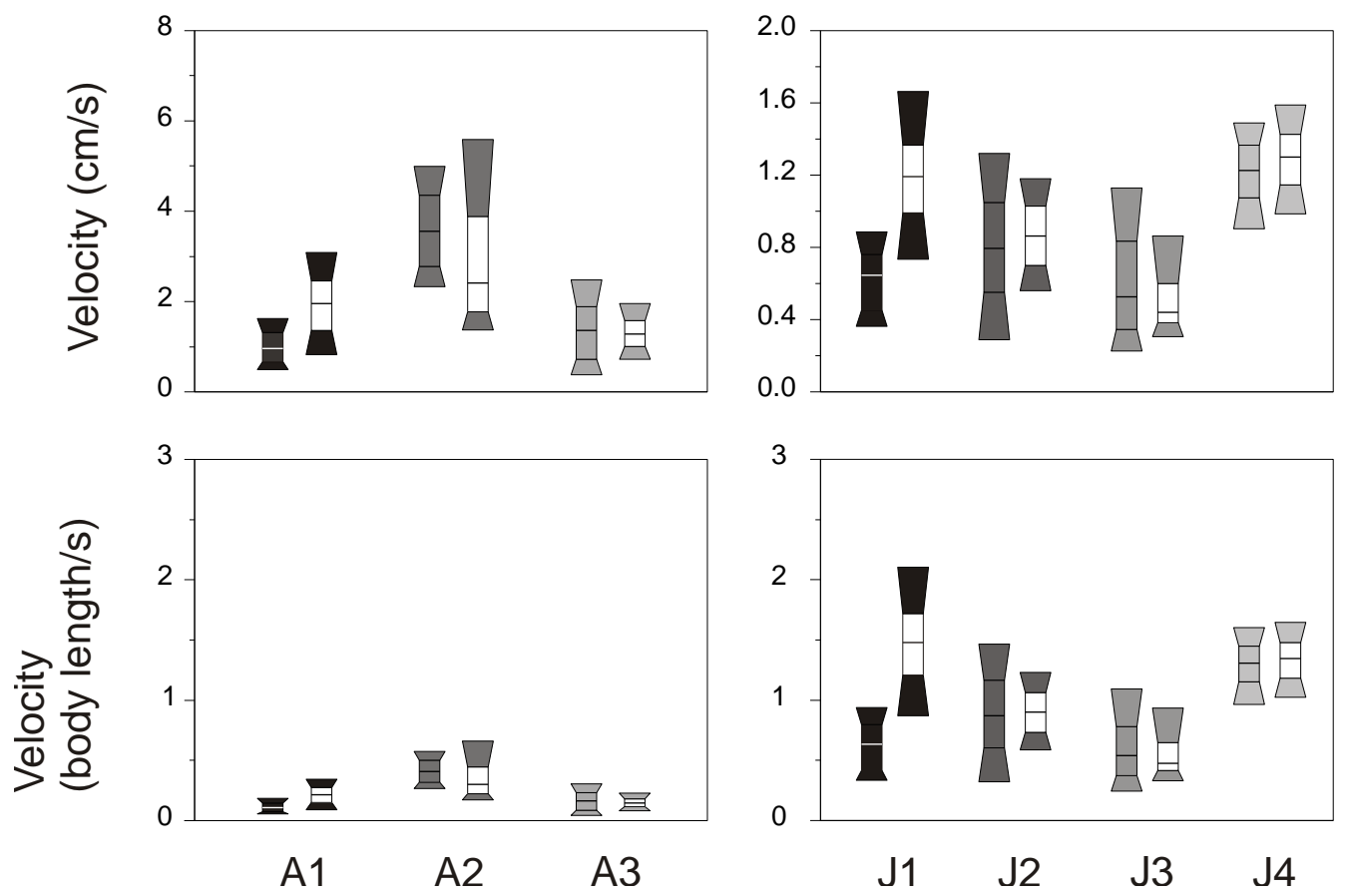

Figure 4.1. Walking velocities for freely walking adult (left column) and juvenile (right column) crayfish with caudal (shaded boxes) and rostral (white boxes) centers of mass. Individual animals (A, adults; J, juveniles) are plotted on the $x$-axis. Box plots show the $10^{\text {th }}, 25^{\text {th }}, 50^{\text {th }}, 75^{\text {th }}$, and $90^{\text {th }}$ percentiles for the distibutions. 


\section{Step variables}

Legs 2 and 5 showed the largest differences in stepping behavior when the center of mass was shifted. Adults showed a shorter period duration in leg 5 compared to clawless adults (Figure 4.2A). Shifting the juvenile center of mass did not show any difference in leg periods (Figure 4.2B). However, both clawless adults and juveniles showed significantly larger stance duration in the fifth legs. Under these same conditions, leg 2 significantly decreased its stance duration.

Both adults and juveniles increased leg swing durations when the center of mass was more caudal. This was true for all adult legs except for leg 3 which showed no significant change. Juveniles also increased swing duration in legs 2, 3, and 4. However, leg 5 showed decreased swing duration. Swing ratios increased in leg 2 and decreased in leg 5 in caudal conditions (Figure 4.2C). Loaded juvenile legs 3 and 4 also showed larger swing ratios.

All legs increased their stride length when the center of mass was caudal-located in both juveniles and adults (Figure 4.2C). Comparing adults to loaded juveniles and clawless adults to juveniles, normalized stride lengths were similar across legs while swing ratios were generally larger in both juvenile conditions (Figure 4.2D).

\section{Spatial orientation of legs relative to body axis and center of mass}

The effects of changing the center of mass on the spatial orientation of the legs were investigated. In adults and loaded juveniles leg 5 excursion lengths were shorter (Figure 4.3, top row). This is due to a more anterior posterior extreme position (PEP) compared to the mean PEP when the center of mass was caudal. Another noticeable difference was observed in the relative spatial orientation of the chelae between adults and loaded juveniles. Extreme positions of adult chelae were more anterior than juveniles relative to the body position. Juvenile extreme positions (legs 2-5) were also slightly offset in the caudal direction relative to adults (Figure 4.3A). Spatial coordinates of the mean AEP for legs 2 and 3 are anterior to the rostrum in adults and posterior to the rostrum in juveniles. The same trend appears in legs 4 and 5, most 


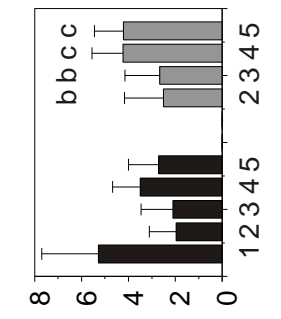

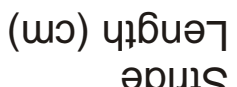

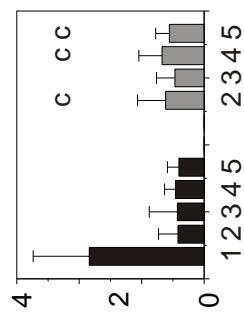

(s) uolpesno bu!̣s

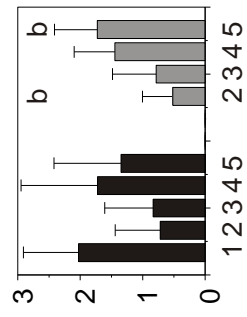

(s) uo!teing

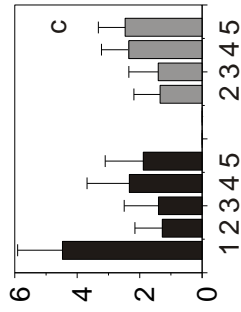

(s) po!̣əd

$\varangle$

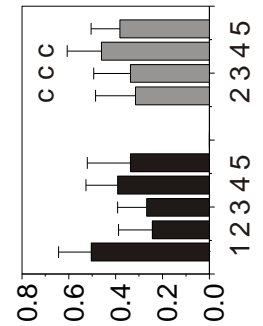

(mว) 41биәา әp!ıtS

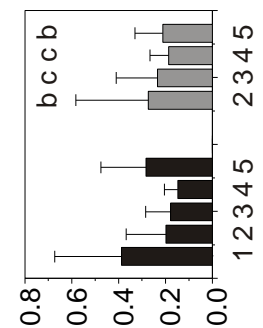

(s) uolpexno buims

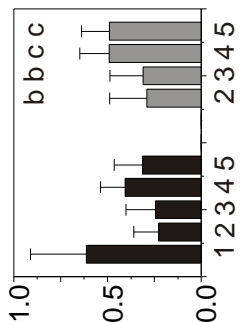

әр!ฺฺS I|np

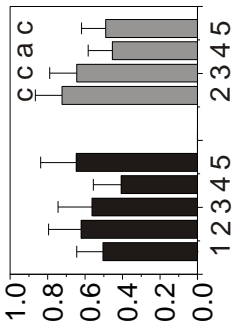

(s) uoliexng әJuetS

O!leג 6u!MS әा!ข^n^

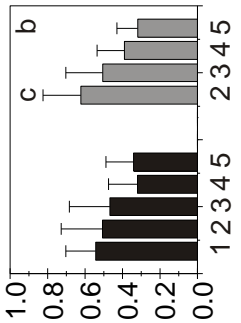

(s) po!lıd

o!̣ed bu!ns IInp $\forall$

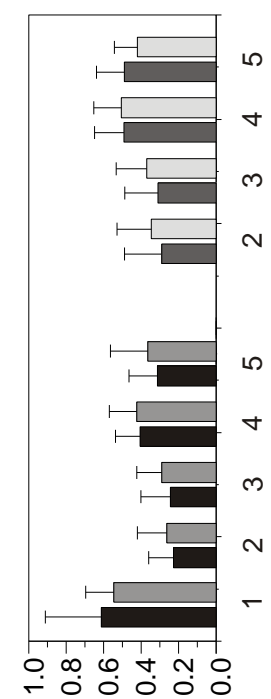

әp!גIS ןEMION

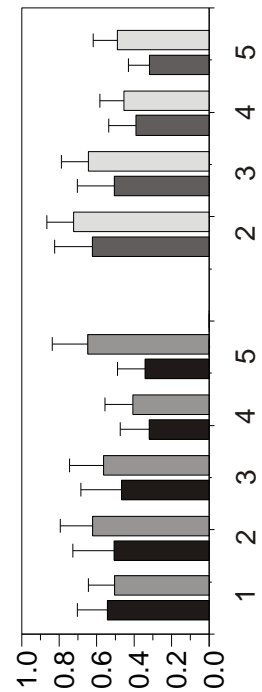

olpey bu!ns

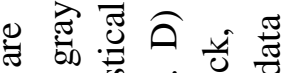

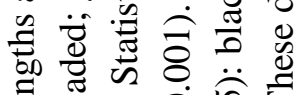

巳

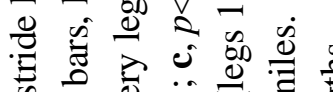

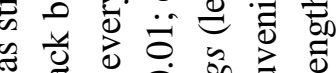

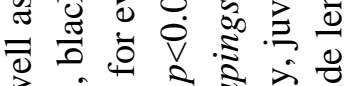

उ

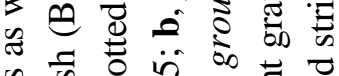

a 包

卷

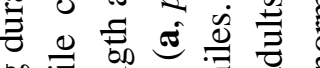

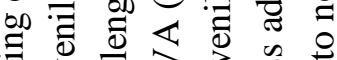

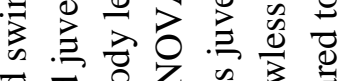

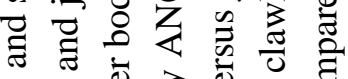

๑

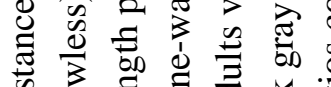

ช் $\frac{\overline{0}}{0}$ 。

की

Q

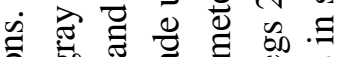

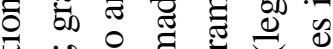

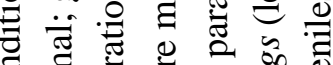

उ

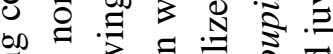

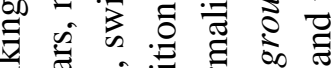

ส

3 पै

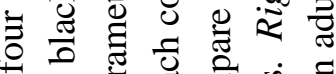

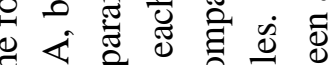

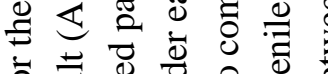

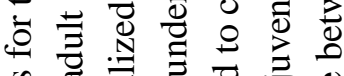

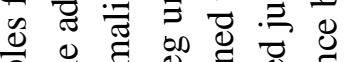

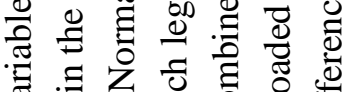

పี $\Xi$ 己

$>$ को $08 \%$ 巳ी仓

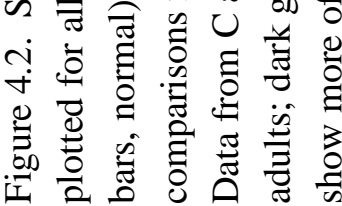


noticeably in the leg 4 PEP and leg 5 AEP relative to the cross-section at the caudal end of the thorax. This finding suggests that there are slight differences in footfall coordinates between juveniles and adults that are not contingent on location of the center of mass. However, it appears that shifting the center of mass causes similar changes to leg 5 footfall coordinates relative to body position of both sizes of crayfish.

Spatial profiles of leg excursions relative to the center of mass describe potential leg combinations that may be used as a base of support. Comparing adult and juvenile profiles reveal a greater change to the juvenile profile after the animals are loaded (Figure 4.3B, bottom row). In juveniles the lateral plane through the center of mass nearly bisects the stance excursion of leg 4. After rostral loading, the center of mass plane remains anterior to the leg 4 excursion and is never crossed by the leg 4 AEP. The rostral shift in juvenile center of mass also results in the stance excursions of legs 2 and 3 to be centered about the center of mass plane. In juveniles the entire excursions of these two pairs of legs remained anterior to the center of mass. With rostral center of mass, the juvenile chelae were the only legs that maintain a complete step that is anterior to the center of mass plane. Furthermore, the distance between leg 5 footfalls and the center of mass increases with the rostral shift in load. These findings suggest that during normal juvenile walking conditions the base of support provided by the legs appears to come from legs 3-4-5. Under loaded conditions the juveniles appear to use legs 1 and 4 as the primary base of support.

Adult profiles showed less change between conditions. Leg 4 stance excursions crossed the lateral plane of the center of mass under both conditions. However, with a caudal center of mass the mean leg 4 PEP was only slightly posterior to the center of mass plane. Under normal conditions, leg 4 AEP was slightly anterior to the center of mass plane with a larger relative posterior displacement of the PEP. Legs 2 and 3 remained anterior to the center of mass during their steps but were shifted closer to the center of mass in intact animals. Leg 5 extreme positions remained posterior to the center of mass during both conditions but increased the posterior displacement of its AEP and PEP in animals with a rostral center of mass. The chelae maintained positions well anterior to the center of mass, more than twice the relative distance observed in the juveniles. It appears that the majority of the base of support is provided by the same legs (3-4-5) during both conditions of adult walking. 
A

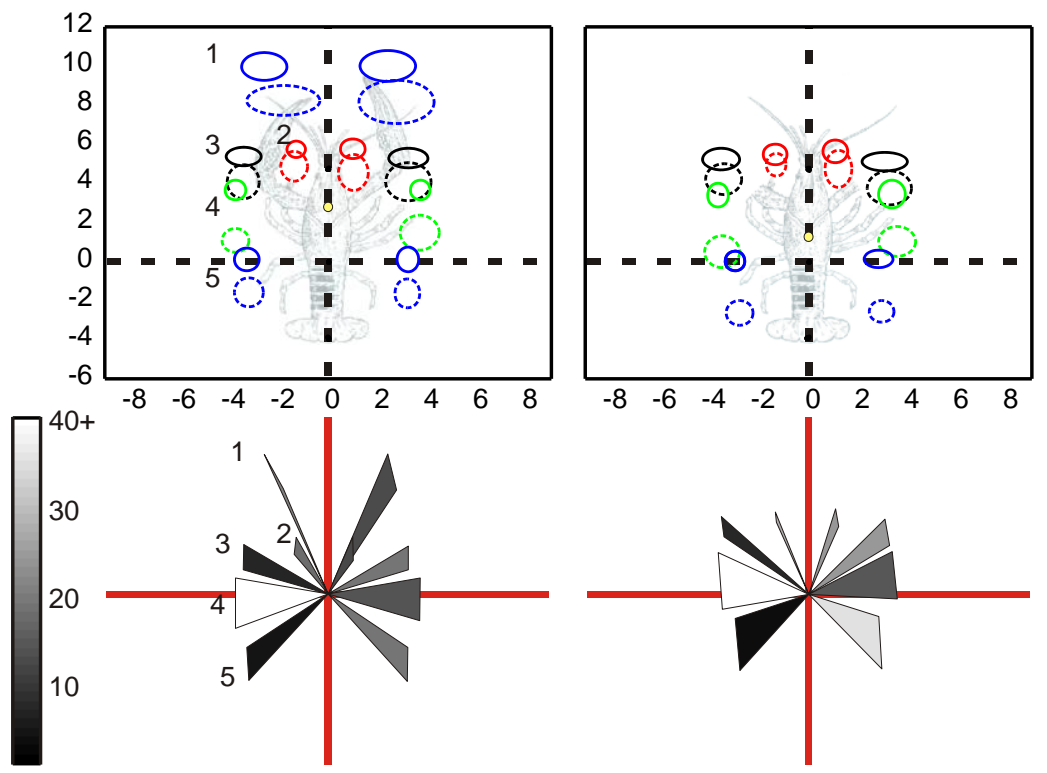

B

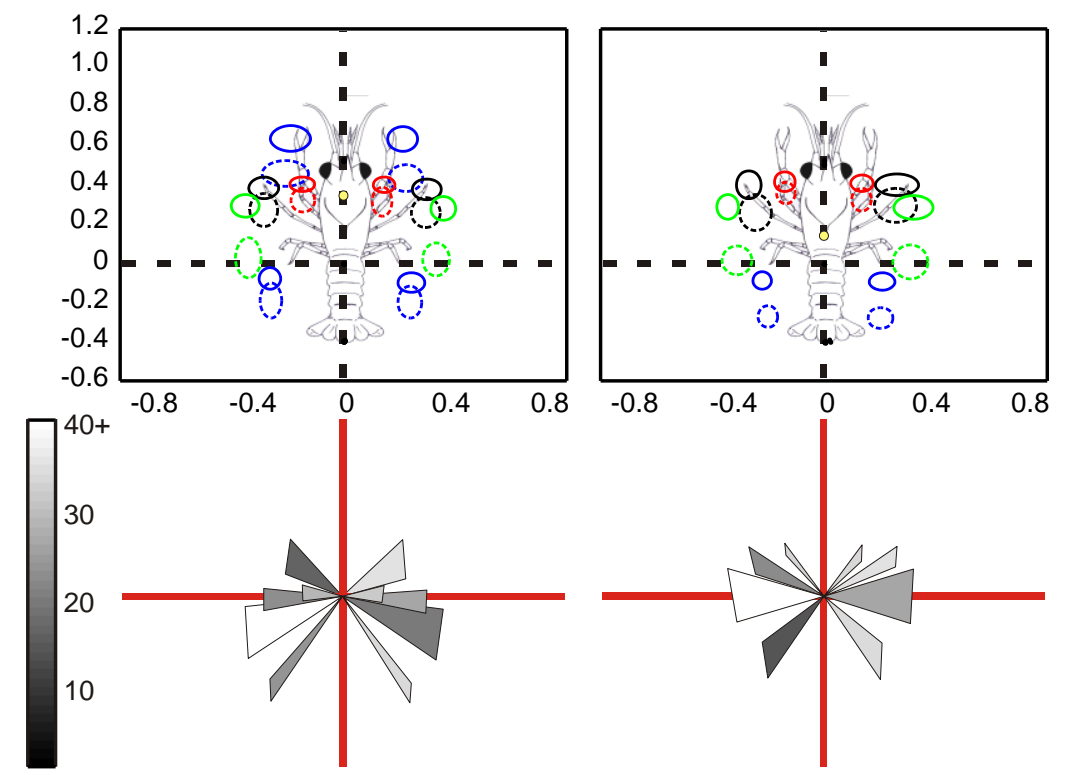

Figure 4.3. Rows 1 and 3: Anterior and posterior extreme positions (AEP, solid lines; PEP, dashed lines; leg 1, blue; leg 2, red; leg 3, black; leg 4, green; leg 5, blue) plotted relative to the body axis and center of mass (yellow circles). A) Adults (left panel, normal; right panel, clawless) and B) Juveniles (left panel, loaded; right panel, normal). Circles are constructed as the standard deviation in the $x$ and $y$ direction around the mean extreme position. Dashed crosshairs represent the intersection of the thorax and abdomen along the dorsal midline (axes in centimeters). Rows 2 and 4: AEPs and PEPs are connected to represent the excursion length between the two mean extreme positions. Extreme positions are oriented to the center of mass location (intersection of the red crosshairs). The colormap represents the percentage of swing shared with the reference leg left 4 (L4). Therefore, legs L3 and L5 very seldom swing at the same time as L4 during adult walking (bottom panels in A). In the juveniles, legs L3 and L5 share a larger percentage of L4 swing, especially in loaded animals (leftt panel, bottom row, B). Scale is the same as the top row. 
Comparing the percentage of swing that legs share provides another perspective as to how legs are coordinated. Swing times were contrasted against leg L4 to determine which legs were likely to move together. In the adults leg 4 least often shared swing with its ipsilateral neighbors L3 and L5 suggesting that these legs were on the substrate while leg 4 was swinging (Figure 4.3A). Adult L4 shared 6\% swing time with L3 and 3\% with L5 during both conditions. Contralateral right legs R3-R4-R5 also showed the lowest shared swing percentages when normalized to R4 (data not shown). Using 15\% shared swing as a determinant, legs L3-L5-R4-R2-R1 were more often in stance while legs L4-L2-L1-R5-R3 were more likely to swing with a rostral center of mass (Figure 4.3A, first panel bottom row). These animals showed angular excursions in legs 1 and 2 extending from the center of mass that were very near (L1-L2) or overlapping (R1-R2) one another. The effect of this spatial orientation with the relative timing of swing makes the adult profile appear much like an alternating tetrapod in which legs 3 and 5 on one side of the body move with legs 4 and 2 (here $2+1$ ) of the contralateral side. Amputating the chelae did not greatly alter the profile of the walking legs. Again using the $15 \%$ criterion, clawless adults resembled an alternating tripod in which legs L3-L5-R4 were more likely to remain in stance while L4-R3-R5 were swinging. The distinction between potential alternating groups of legs is more discernible in clawless adults when the center of mass is in a more caudal position. Legs L2 and R2 both share a high percentage of swing with L4. These legs also share similar swing percentages with R4. The variability suggested by these results coupled with the large swing ratios observed in legs L2 and R2 indicates that these legs may not play a large role in postural support when the center of mass is shifted farther from these legs.

Juvenile profiles resembled clawless adults. However, shared swing percentages were larger in all legs. Ipsilateral L5 shared a larger percentage with L4 (13\% in normal juveniles, $3 \%$ in adults), but was still the lowest percentage observed across all juvenile legs (Figure 4.3B, bottom row). The same relationships held true for right ipsilateral legs. The difference between adults and juveniles may be attributed to the higher walking velocities in juveniles. Due to this general increase in shared swing across all legs, the criterion for qualifying like groups of juvenile legs was adjusted to $26 \%$ shared swing. Under this scenario legs L3-L5-R4 were most likely to occupy stance while L4 was swinging. Legs 2 also behaved much like clawless adult legs 2. Therefore, at times it appears that normal juvenile walking also resembles an alternating tripod. However, juvenile shared swing percentages were relatively higher between legs 3 and 4 (22\% 
and 29\%, left and right legs, respectively) compared to legs 4 and 5 (13\% and 12\%, left and right legs, respectively). The higher shared swing percentage between ipsilateral legs 3 and 4 suggests that juveniles walk more like four-legged walkers with L4-R5 and R4-L5 acting as a base of support.

Loaded juveniles showed spatial and swing profiles different from any of the other three conditions. Following the $26 \%$ criterion, legs show potential groupings of L1-L3-L5-R4 and L2L4-R1-R2-R3-R5. The lowest shared swing percentages relative to L4 were found in legs L1 and R4 (16 and 19\%, respectively). The spatial profile describing limb orientation in loaded juveniles shows that legs 1 and 4 excursion lengths cover more distance than the remaining legs (Figure 4.3B, top row). Data presented earlier show that legs 1 and 4 have much longer stance durations compared to the other legs. These three results would suggest that the base of support in the loaded condition comes primarily from alternating leg pairs L1-R4 and R1-L4.

\section{Ipsilateral coordination}

Juveniles with a caudal center of mass displayed strong alternating coordination between legs 4 and 5 (Figure 4.4). This relationship was completely diminished when loaded. In adult legs 4 and 5 a rostral center of mass lowered the occurrences of alternating coordination and broadened the phase distribution but it did not have as strong effect as in juveniles. In juveniles, leg 4 predominantly steps in the first half of the leg 3 step when the center of mass is caudal. A rostral shift in center of mass shows a wide distribution that peaks between 0.4-0.6 phase values. In adults with a rostral center of mass the leg 4 in 3 phase relationship shows a larger peak distribution between 0.4-0.6. With a caudal center of mass, the adult 4 in 3 relationship is more variable. Coordination between adult and juvenile ipsilateral legs 2 and 3 showed very little change after a shift in center of mass.

Contralateral coordination was highly variable in all juvenile leg pairs under both conditions. The fifth pair of legs showed the highest frequency of coordination of all legs when center of mass was caudal (20.5\% frequency at $0.4 \pm 0.05$ phase value) (Figure 4.5$)$. The fourth legs were the most coordinated with a rostral center of mass ( $20.0 \%$ occurrence at $0.7 \pm 0.05$ phase value). 

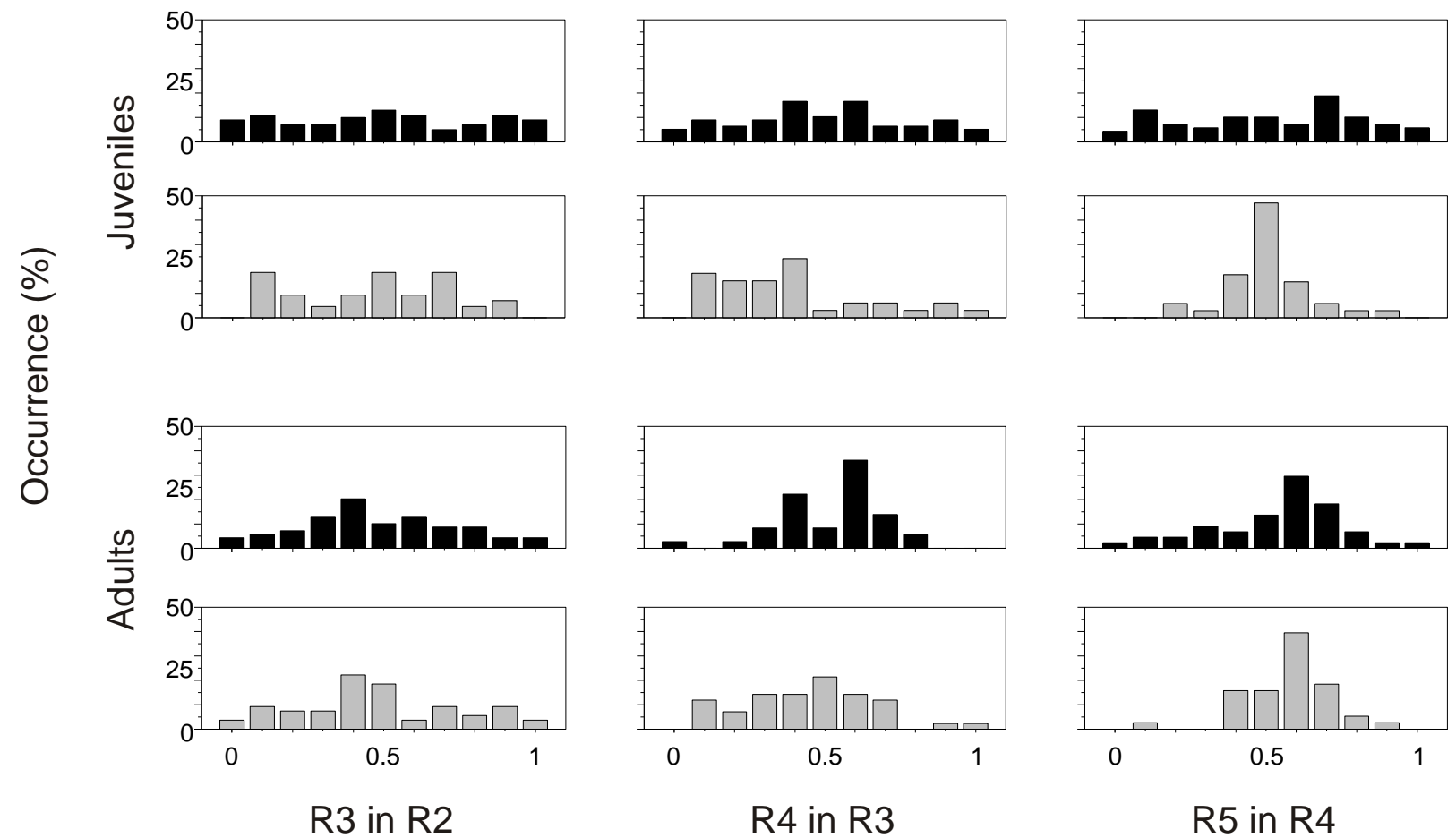

$\mathrm{R} 4$ in $\mathrm{R} 3$

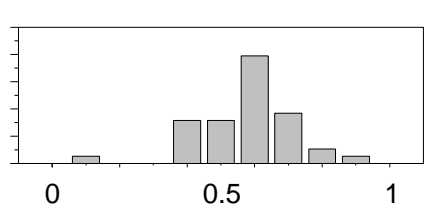

R5 in R4

Figure 4.4. Phase distributions of ipsilateral legs. The top two rows show ipsilateral phase distributions in juveniles (loaded, black; normal, gray). The bottom two rows show adult phase distributions (normal, black; clawless, gray). 


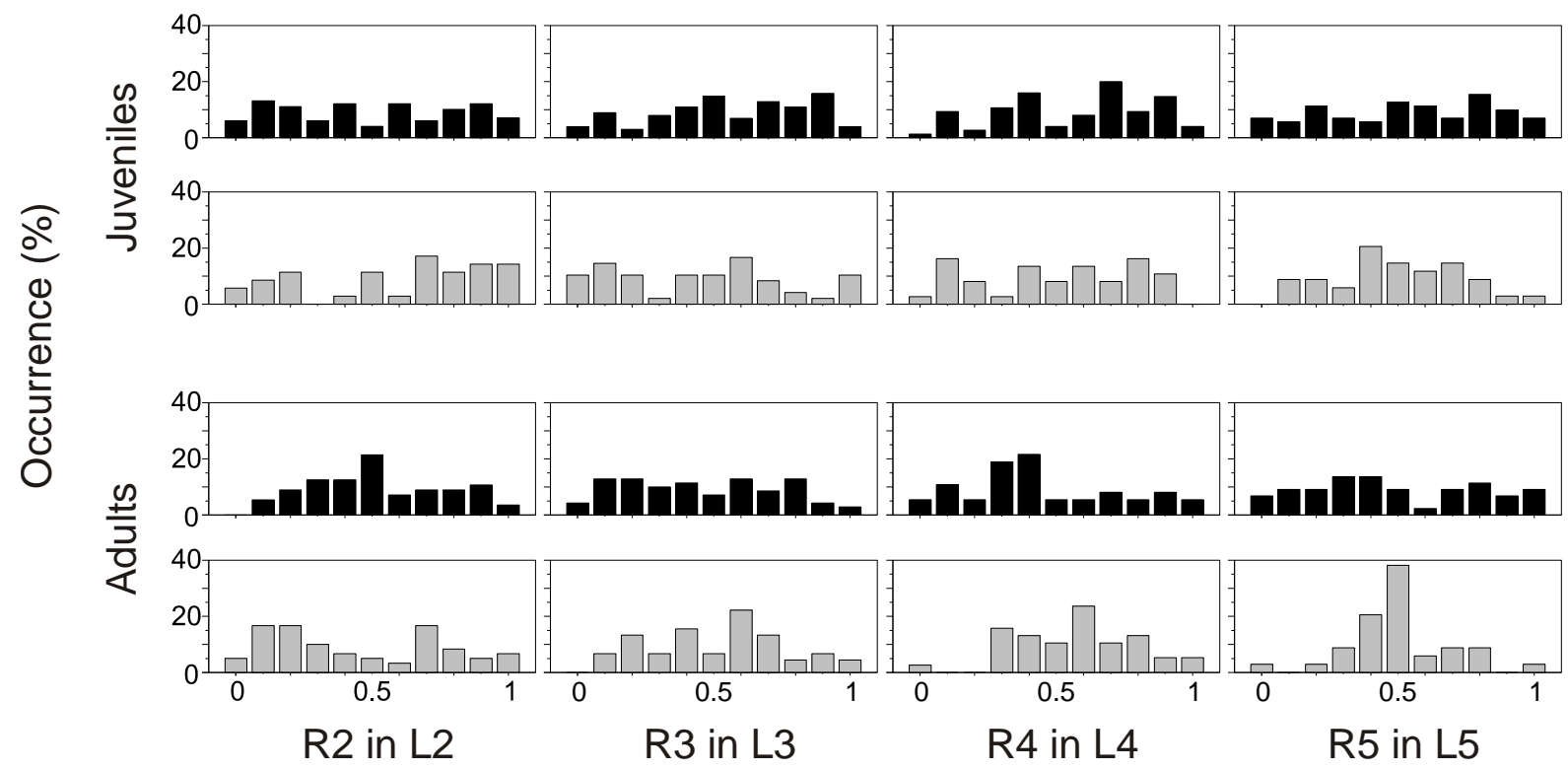

Figure 4.5. Contralateral phase relationships in juveniles (top rows; loaded, black; normal, gray) and adults (bottom rows; normal, black; clawless, gray). 

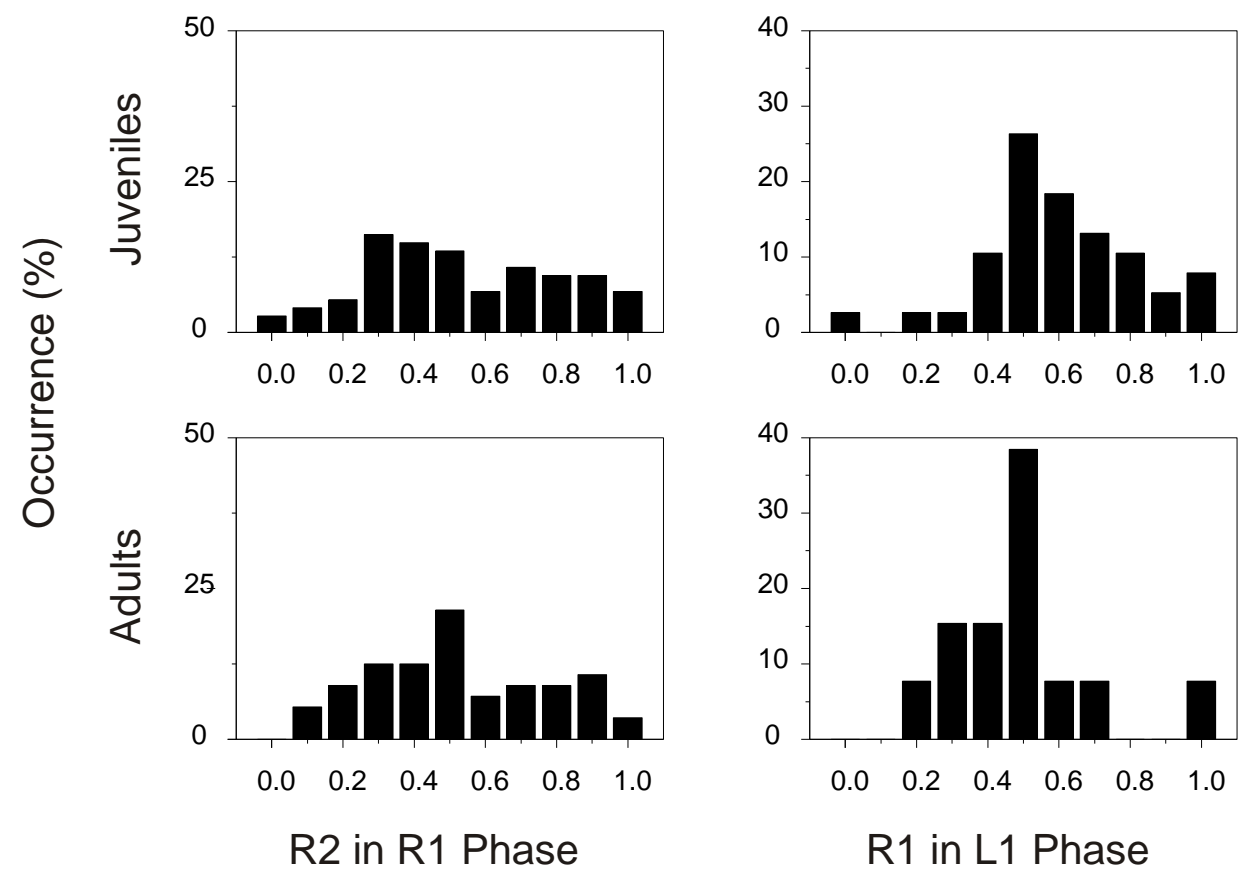

R1 in L1 Phase

Figure 4.6. Ipsilateral (left column) and contralateral (right column) phase relationships of juvenile (top row) and adult (bottom) chelae during walking trials with a rostral center of mass. 
Adults showed the same trend with less variability. The fifth legs showed more frequent alternating coordination with a caudal center of mass. A rostral center of mass resulted in leg four phase relationships with $48.6 \%$ of the steps in a $0.25-0.45$ phase range.

Adults and loaded juveniles used the chelae during walking. Two of the three adults (A1 and A3) as well as all loaded juveniles included the chelae in the stepping pattern. The resulting coordination was predominantly alternating between left and right chelae (Figure 4.6).

\section{Limb kinematics}

Kinematics were characterized at four proximal joints for both conditions in adults. Figure 4.7 shows an example of joint kinematics during a walking trial (clawless adult, A2). Of all the legs, leg 5 showed the most consistent trajectories across all four joints. Joints in leg 5 typically displayed the largest ranges of motion.

All steps were normalized to periods and joint angles were averaged for the adult trials. Legs 4 and 5 displayed the largest ranges of motion in the TC, CB and MC joints (Figure 4.8). Leg 5 joint angle trajectories were most affected by a shift in center of mass. A rostral center of mass reduced the ranges of motion in these joints. The IM joint, which is mostly inactive, showed displacement in leg 5 with a caudal center of mass.

\section{Joint angular velocities}

Angular velocities of the leg 5 joints are also affected by the location of the center of mass. The highest velocities are observed during the swing phase of a step. In leg 5 the mean peak angular velocities occur when there is a caudal center of mass (Figure 4.9). This increase was observed in all four of the joints. The three proximal joints in leg 4 are unaffected by the location of the center of mass. However, it appears that a rostral center of mass increases the peak angular velocity in the MC joint. The proximal joints in legs 2 and 3 show greatly reduced velocities 


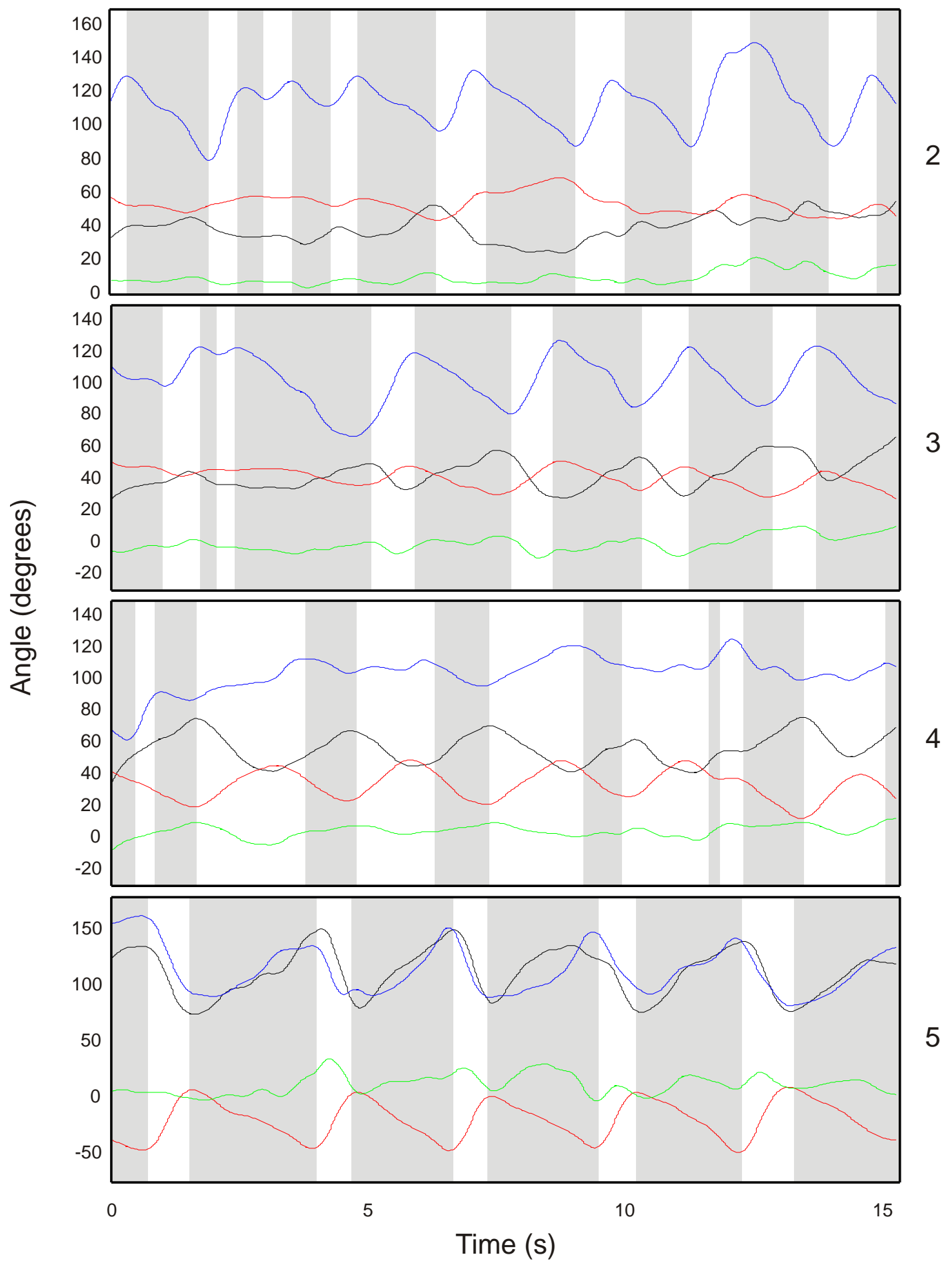

Figure 4.7. Kinematics of four joints in the walking legs during one trial (animal A2, clawless adult). The legs are plotted anterior to posterior (2-5, top to bottom). Joints: TC, black; CB, red; IM, green; MC, blue. Shaded area: stance phase. 
TC

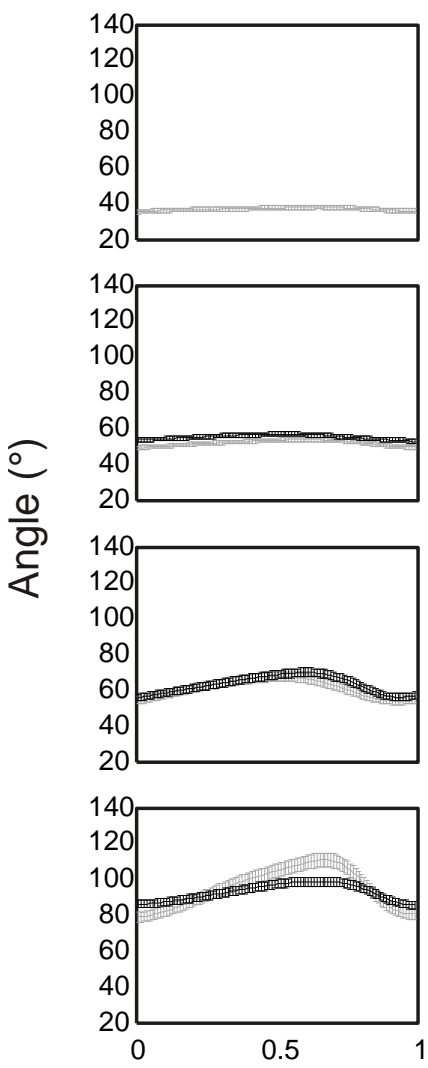

$\mathrm{CB}$
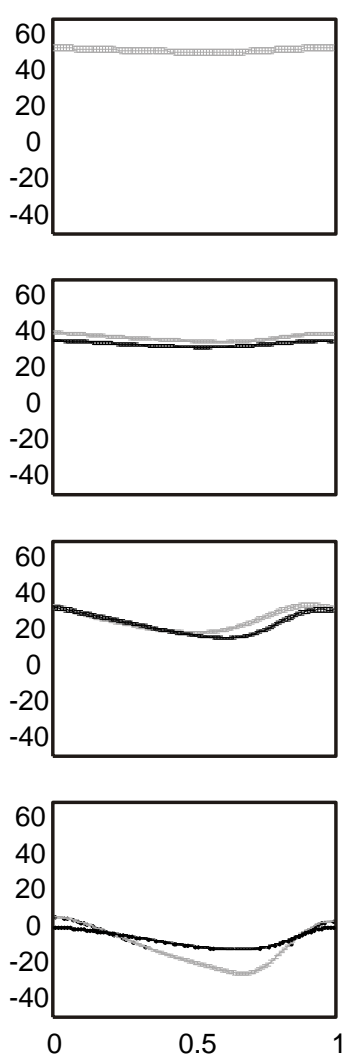

IM
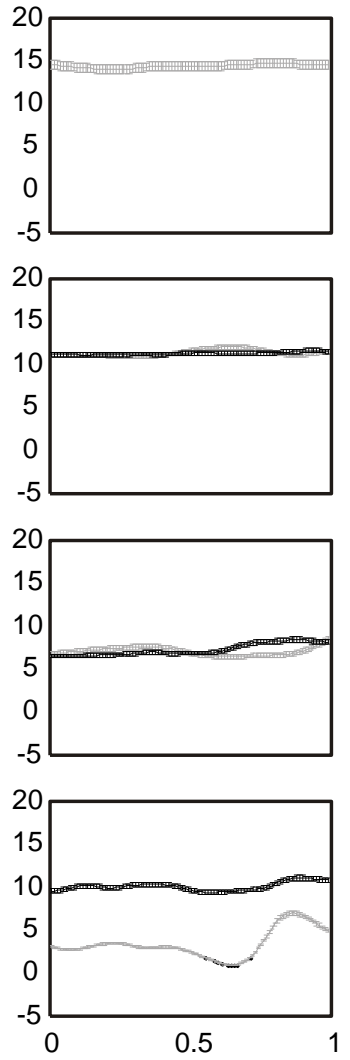

$\mathrm{MC}$

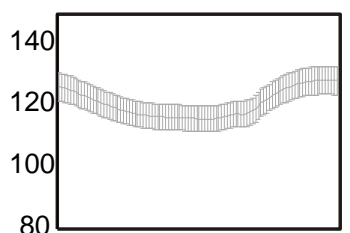

2

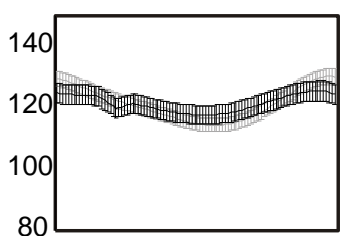

3

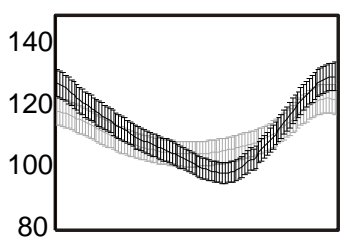

4

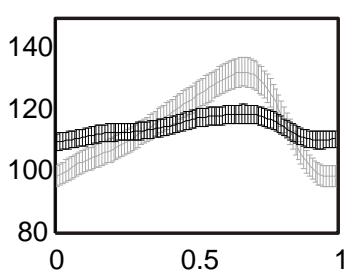

Figure 4.8. Mean angles show that joint kinematics in leg 5 change with a caudal shift in center of mass. Data are averaged for all adult trials with chelae (normal, black \pm s.e.m.) and without chelae (clawless, gray \pm s.e.m.) and plotted as a normalized step ( 0 represents onset of stance and 1 represents the end of swing). Columns (left to right): TC, CB, IM, MC. Leg 2 was often obstructed when the chelae were intact; therefore, few data were gathered for TC and CB and no data were obtained for IM and MC. 
TC
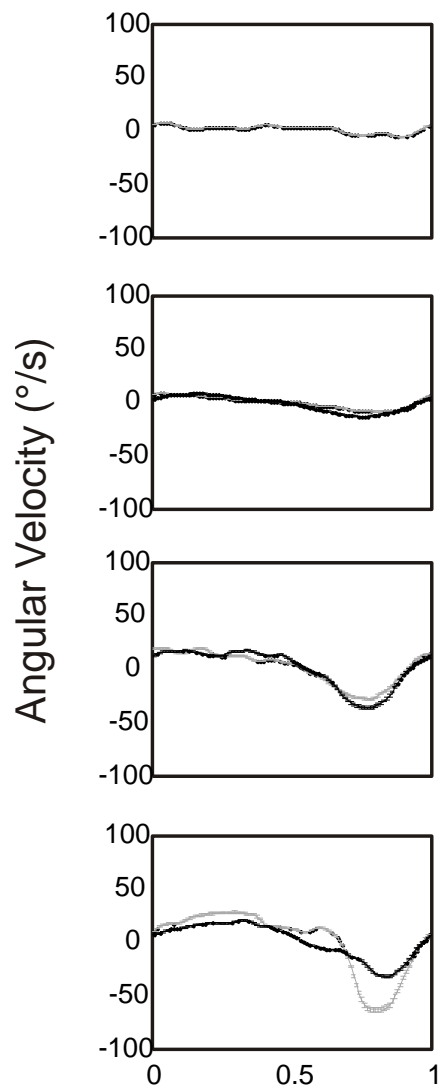

CB
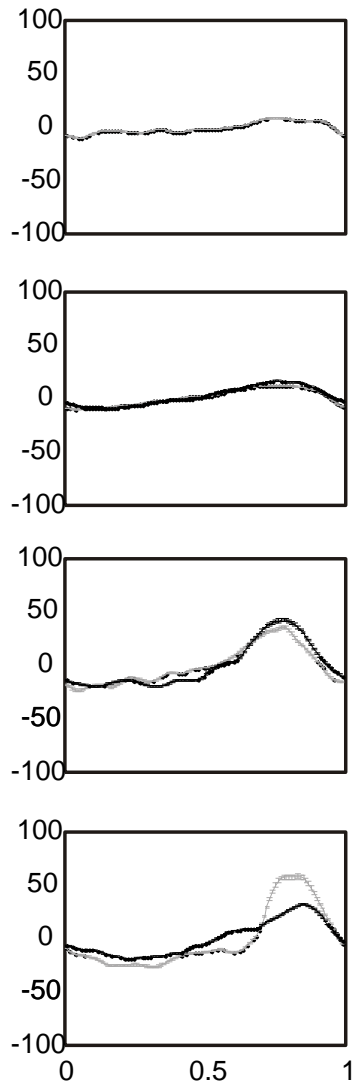

IM
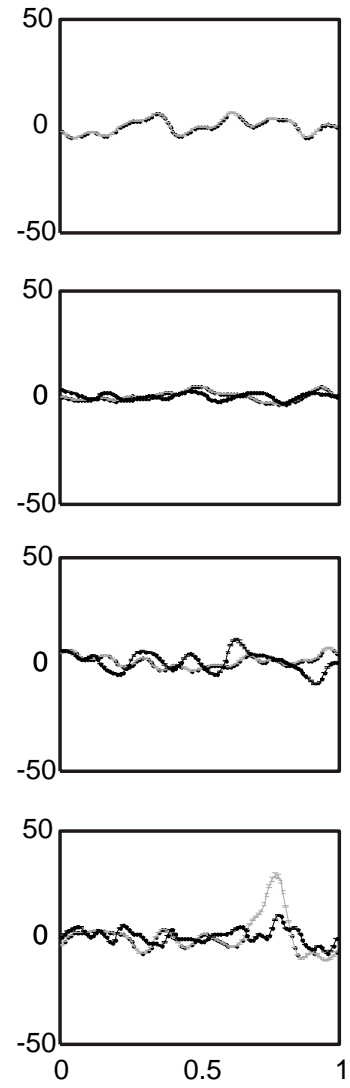

MC

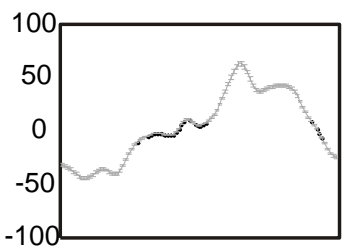

2

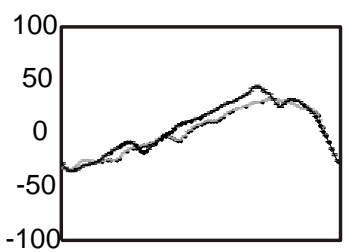

3

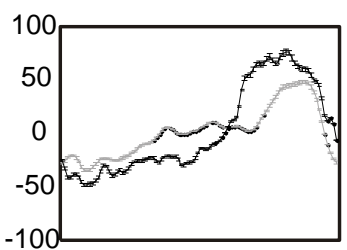

4

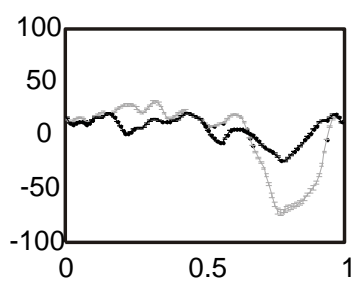

\section{Normal Step}

Figure 4.9. Angular velocities of the four proximal joints in the adult walking legs (normal, black; clawless, gray). Angular velocity during the swing phase of each joint increases in leg 5 and decreases in leg 4 (TC and MC) when the center of mass is in a more caudal location. Joints, TC and MC, in leg 4 show a slight but significant decrease in the swing velocity when center of mass is located caudally. Data are plotted as the mean angular velocity \pm s.e.m. 
during both conditions. The MC joints in these legs display peak velocities comparable to legs 4 and 5. However, the slope of the increase is flatter than legs 4 and 5. This suggests that the angular acceleration is not as fast during the beginning of the swing phase for legs 2 and 3 .

\section{Discussion}

Crayfish show different limb behaviors and stepping patterns depending on the location of their center of mass. This occurs regardless of body size or age. It has been shown that juvenile and adult crayfish display differences in walking behavior (Jamon and Clarac, 1995; Chapter 2). However, adult crayfish used in this experiment have shown that some aspects of walking behavior at very slow velocities more resemble juvenile behavior. Adults displayed lower swing ratios and longer stance durations in posterior legs 4 and 5 similar to juveniles which walk at relative speeds over four times faster. This contradicts previous findings in adult Procambarus which showed these animals displayed more uniformity in behavior of all limbs (Jamon and Clarac, 1995). Those animals used a goal-oriented behavior whereas the adults in this study were likely showing exploratory walking behavior. The goal-oriented animals showed walking speeds much higher than those recorded here (Jamon and Clarac, 1995). Jamon and Clarac (1995) also report no instances of their crayfish using the chelae in the stepping pattern. Therefore, it appears that during slow exploratory walking crayfish will use their chelae. Forward walking soldier crabs (Mictyris platycheles) have been shown to use their chelae when walking at slower speeds (Sleinis and Silvey, 1980). This could have a direct influence on the anterior legs 2 and 3 if the chelae are relieving some of the load on these legs. The fastest walker observed here (A2) did not walk with her chelae.

Juveniles also used their chelae during walking but only with a rostral center of mass. It appears that a rostral center of mass in juveniles produces a unique gait in which the primary base of support comes from the chelae and the fourth pair of legs. This is likely due to a center of mass that is more anterior to the AEP of the walking legs. As mentioned above for adults, use of the chelae during walking could reduce the effects of increased load on anterior legs 2 and 3. However, both juveniles and adults showed a significant increase in leg 2 stance durations when the center of mass was rostral. Conversely, a caudal center of mass resulted in a significant 
increase in leg 5 stance durations. Therefore, relative changes in load on individual legs can affect how that leg functions during a step.

Posterior legs change coordination with the location of the center of mass. Ipsilateral legs 4 and 5 as well as contralateral legs 5 show higher frequencies of alternating coordination when the center of mass is closer to them. A rostral center of mass reduces this coordination but enhances it in ipsilateral legs 3 and 4 and contralateral legs 4 . When the chelae are used in the stepping pattern with a rostral center of mass, they also show an alternating coordination. An increase in the total load has been shown to increase bouts of alternating coordination in contralateral leg pairs 2-5 (Barnes, 1977; Grote, 1985; Clarac and Barnes, 1985). The results presented here suggest that individual legs can change their coordination with neighboring legs when changes in load are localized.

Center of mass affected the kinematics of leg 5 joints. Joint ranges of motion were reduced the farther away the center of mass. This suggests that an increase in load on a leg produces a change in the motor output of that leg. There may be a mechanism that changes whole-limb function by increasing stance duration when load on a leg increases. Such a mechanism may be mediated by cuticular stress detectors (CSD) or the funnel canal organ (FCO). Inputs from these load receptors have been shown to alter stance-swing transitions within individual legs (Klärner and Barnes, 1986; Libersat et al., 1987). Each of the proximal segments shows an increase in its angular displacement. However, leg 4 shows slight changes only in the MC joint when the center of mass moves. Therefore, a second potential mechanism may be located in individual segments within a leg. Muscle and tendon receptor organs measure changes in length and contractile strength of individual muscles (Duysens et al., 2000). It may be possible that the observed changes in limb behavior are a culmination of segmental reflexes responding to load.

Load-mediated reflexes can explain increases in joint displacement during stance phase. However, an increase in load on a leg also produces changes to the joints during swing phase. When leg 5 is nearer the center of mass, angular velocities of the joints increase during the swing phase but remain constant during the stance phase. Because there is no load on the leg during the swing portion of the step, it is unlikely that load receptors directly influence swing velocities. More likely, proprioceptors that respond to joint displacement, called chordotonal organs (COs), 
are involved (Cattaert and Le Ray, 2001). If load increases angular displacement during stance, COs can measure the resulting segmental displacement and produce a reflex relative to the amount of that displacement (Clarac et al., 1978). Faster swing velocities would be beneficial in maintaining posture for an animal that is experiencing large loads to given legs.

In summary, moving the center of mass produces changes in behavior of individual limbs similar to loading the entire animal (Barnes, 1977; Grote, 1981; Clarac and Barnes, 1985). Posterior limbs respond to local increases in load by changing their kinematics. More stereotyped coordination results in those legs that are closest to the center of mass. If legs use isolated reflex mechanisms to accommodate these changes, coordinating mechanisms between legs may not be necessary. This appears to be the case in cockroaches which can detect load transfer between neighboring ipsilateral legs (Zill et al., 2009). A similar mechanism could explain how crayfish during ontogeny deal with a change in center of mass with nervous systems that are functionally the same (Chapter 3).

\section{References}

Barnes, W. J. P. (1977). Proprioceptive influences on motor output during walking in the crayfish. J. Comp. Phsiol. (Paris) 73, 543-564

Borgmann, A., Scharstein, H., and Büschges, A. (2007). Intersegmental coordination: Influence of a single walking leg on the neighboring segments in the stick insect walking system. $J$. Neurophysiol. 98, 1685-1696

Cattaert, D. and Le Ray, D. (2001). Adaptive motor control in crayfish. Progress in Neurobiology. 63, 199-240

Chrachri and Clarac, F. (1990). Fictive locomotion in the fourth thoracic ganglion of the crayfish, Procambarus clarkii. J. Neurosci. 10(3), 707-719

Clarac, F. and Barnes, W. J. P. (1985). Peripheral influences on the coordination of the legs during walking in decapods crustaceans. In: Coordination of motor behavior. Bush, B.M.H. and Clarac, F. (eds.). Cambridge University Press, Cambridge. pp 249-269 
Clarac, F., Vedel, J.P. and Bush, B.M.H. (1978). Intersegmental reflex coordination by a single joint receptor organ (CB) in rock lobster walking legs. J. exp. Biol. 73, 29-46

Cruse, H. (1990). What mechanisms coordinate leg movement in walking arthropods? Trends Neurosci. 13, 15-21

Cruse, H. and Müller, U. (1984). Short communications: A new method measuring leg position of walking crustaceans shows that motor output during return stroke depends upon load. J. exp. Biol. 110, 319-322

Duysens, J., Clarac, F. and Cruse, H. (2000). Load-regulating mechanisms in gait and posture: comparative aspects. Physiological Reviews. 80, 83-133

Grote, J. R. (1981). The effect of load on locomotion in crayfish. J. exp. Biol. 92, 277-288

Hughes, G. M. (1952). The coordination of insect movements. I. The walking movements of insects. J. exp. Biol. 29, 267-284

Jamon, M. and Clarac, F. (1995). Locomotor patterns in freely walking crayfish (Procambarus clarkii). J. exp. Biol. 198, 683-700

Klärner, D. and Barnes, W. J. P. (1986). The cuticular stress detector (CSD2) of the crayfish. II. Activity during walking and influences on leg coordination. J. exp. Biol. 122, 161-175

Libersat, F., Clarac, F. and Zill, S. (1987). Force-sensitive mechanoreceptors of the dactyl of the crab: single-unit responses during walking and evaluation of function. J. Neurophysiol. 57, $1618-1637$

Müller, U. and Cruse, H. (1991a). The contralateral coordination of walking legs in the crayfish Astacus leptodactylus. I. Experimental results. Biol. Cybern. 64, 429-436

Sillar, K. T., Clarac, F. and Bush, B. M. H. (1987). Intersegmental coordination of central neural oscillators for rhythmic movements of the walking legs in crayfish. Pasifastacus leniusculus. J. exp. Biol. 131, 245-264 
Sleinis, S. and Silvey, G. (1980). Locomotion in a forward walking crab. J. Com. Phsiol. 136, $301-312$

Zill, S.N., Keller, B.R., Duke, E.R. (2009). Sensory signals of unloading in one leg follow stance onset in another leg: Transfer of load and emergent coordination in cockroach walking. $J$. Neurophsiol. 101, 2297-2304 
Chapter V: Modeling crayfish locomotion in a real-world environment reveals walking as an emergent property 


\begin{abstract}
Successful locomotion requires complex interactions between an environment and an organism. During walking, legs must produce forces necessary for propulsion in the desired direction of movement while also producing forces to counteract gravity to support the animal in the vertical plane. In multi-legged systems it appears that behavior is not always uniform across legs and limb function depends on location relative to the body. Crayfish leg behaviors can change depending upon their proximity to the animal's center of mass. The neural circuitry underlying this flexible control in crayfish is unknown. I have designed an eight-legged model crayfish with a simplified neuromechanical system to test exactly how much, or how little, control is required by an animal to accommodate changes in center of mass and produce successful locomotor behavior. Control in the model nervous system is isolated to loops in individual legs. Kinematic movements are identical in all legs when no feedback is present. The model lacks any interlimb neural circuitry and does not use any intrinsic coordinating mechanisms between legs. The only feedback from the environment necessary to produce realistic walking behavior comes from contact sensors at the end of the legs which influence leg central pattern generators. As a result, walking behavior arises from indirect mechanical coupling of the legs through the environment and body of the animal. The model produces realistic gaits and adapts to changes in load distributions and limb amputations the same as actual crayfish. These data suggest that animals do not need complex control mechanisms because they already exist in the form of biomechanical interactions.
\end{abstract}

\title{
Introduction
}

Crayfish respond to changes in load distribution at the level of individual legs (Chapter 4). This finding prompted the question as to how tightly coupled interlimb communication might be. Changes in center of mass can be controlled in the animals but neural circuitries involved in motor control have yet been identified. Therefore, modeling a crayfish with realistic body design and a simplified neuromechanical system makes it possible to test for the minimal amount of neural control necessary to produce the observed changes in behavior that occurs with different center of mass locations. 
Limb movements can be driven by oscillating neural networks in the central nervous system without sensory feedback (review by Delcomyn, 1980). These oscillating networks are known as central pattern generators, or CPGs. Stick insects appear to have a CPG associated with each leg joint (Büschges et al., 1995; Bässler and Büschges, 1998). In crayfish, studies on isolated nerve cords have shown output from the oscillatory network can be recorded in motor neurons of the proximal-most leg segments (Sillar et al., 1987; Chrachri and Clarac, 1990). The rhythm of oscillators of a leg as well as adjacent ipsilateral ganglia can be entrained by sensory feedback (Sillar et al., 1987). Rhythmic output from these ganglia suggests that ipsilateral oscillators may be neurally coupled, but they fire in phase with each other when feedback is reduced or eliminated (Borgmann et al., 2007). This is opposite to what is seen in intact animals, which show alternating coordination between neighboring legs (Hughes, 1952). Ipsilateral limbs show such strongly coupled movements that it is often assumed that legs must communicate with one another (Cruse, 1990; Cruse et al., 2007). However, analysis of walking in juvenile and adult crayfish has suggested that ipsilateral coupling of legs may not be as highly controlled as previously supposed. These animals showed more variable ipsilateral coordination in limbs farther from the center of mass (Chapter 4).

Contralateral coupling between legs is more variable than between ipsilateral legs (Müller and Cruse, 1991a; Jamon and Clarac, 1995; Chapters 2-3). Contralateral oscillators show no coordinated pattern of activation in the absence of sensory feedback and are thought not to be coupled through the nervous system (Sillar et al., 1987). However, increasing load on a crayfish influences contralateral leg pairs toward a predominantly alternating rhythm (Barnes, 1977; Grote, 1981). When load distribution changes, contralateral legs nearest the center of mass show higher occurrences of alternating coordination (Chapter 4). These findings suggest that load affects coordination between legs that may not be neurally coupled. However, there is no direct evidence to support this idea. One aim of this study is to determine if, and how, limb coordination is dependent on an increase in load feedback to individual legs and to what extent the nervous system is needed.

Stepping patterns can also change when an animal loses a limb, as the remaining intact neighboring legs adopt an alternating coordination (Barnes, 1977; Clarac and Chasserat, 1979; Chapter 3). These results argue against strong neural coupling between legs unless an additional 
circuit exists to carry a signal between and past thoracic ganglia. This would suggest that a mechanism exists to re-coordinate limbs after an amputation event. This seems like an inefficient solution because of the extra circuitry devoted to this specific event. If the model is efficient and robust, the neuromechanical control system should account for limb amputations.

A previous model describing limb coordination in cockroaches uses mutual inhibition between legs as a mechanism to produce walking stability (Beer, 1990). The result is a highly stereotyped alternating tripod gait in a six-legged system. Other models based on stick insect walking (Kindermann et al., 2002; Cruse et al., 2000; Cruse et al., 2002) do not use a centralized walking program, but instead legs are coupled based on a set of coordinating mechanisms (Cruse, 1990). All of these models are highly adaptive and produce robust behavior but they still rely on strict interlimb communication. In the model crayfish presented here, there is no neural coupling between legs. Therefore, there is no communication or coordinating mechanism between legs. This model tests the hypothesis that neither a master neural code nor interlimb coordinating mechanisms are necessary to produce realistic behavior. Successful model walking would show that behavior arises from collective parts rather than from pre-programmed control (Chiel and Beer, 1997).

\section{Methods}

\section{Simulation Software}

The simulation software AnimatLab (Cofer et al., 2006) was used to create a multi-legged animal to test the hypothesis. A model animal with body morphology and behavioral plan was designed based on a crayfish (Procambarus clarkii). Walking simulations in AnimatLab are run within a physics engine, Vortex ${ }^{\mathrm{TM}}$, licensed by CM-Labs, Inc. The physics engine allows for specialized control over real-world parameters such as hydrodynamics and gravity within the virtual environment. The software also allows for discrete control over the location of the center of mass and body density of the model crayfish. 


\section{Body design of the model crayfish}

The body plan of the model crayfish is based on that of a large-bodied P. clarkii. The body is a $15 \mathrm{~cm}$ long cylinder with a radius of $1 \mathrm{~cm}$ and a density of $1.2 \mathrm{~g} / \mathrm{cm}^{3}$, resulting in a total body mass of nearly $60 \mathrm{~g}$.

Design and orientation of the limbs is greatly simplified in the model. Eight identical legs were oriented with bilateral symmetry. The eight legs are referenced as legs $2-5$ with a left and right designation (L2 - R2, L3 - R3, etc.). All legs connect to the ventral side of the body in the same plane. Spatial orientation of the legs is arranged so that ipsilateral legs have a distance of $2.4 \mathrm{~cm}$ between them. The legs are offset rostral to the longitudinal midpoint of the body by $3 \mathrm{~cm}$. Therefore, leg 2 inserts with the body at a distance of $6.6 \mathrm{~cm}$ from the midpoint in the rostral direction, leg 3 at $4.2 \mathrm{~cm}$, leg 4 at $1.8 \mathrm{~cm}$, leg 5 at $-0.6 \mathrm{~cm}$ (Figure 5.1). Limbs were space based on relative dactyl contact points with the substrate in real animals (Vídal-Gadéa et al., 2009).

Each limb is constructed of three segments (analogous to the coxopodite, meropodite, and carpopodite). Limb segment dimensions are based on mean lengths and densities of $P$. clarkii legs. The proximal coxopodite segment is $0.5 \times 0.3 \times 0.25 \mathrm{~cm}$ (height $\mathrm{x}$ width $\mathrm{x}$ length). The meropodite segment is $0.5 \times 0.5 \times 2.85 \mathrm{~cm}$ and the carpopodite is $0.3 \times 0.3 \times 2.8 \mathrm{~cm}$. Each segment also has a defined starting orientation with respect to the body. The coxopodite has a null position orthogonal to the body axis and the dorso-ventral plane. The null position of the meropodite is offset by $15^{\circ}$ toward dorsal. The carpopodite has a $100^{\circ}$ orientation relative to the meropodite (Figure 5.1). All leg starting positions are based on measurements of standing animals (Vídal-Gadéa et al., 2009).

Each limb has three major contributing joints, two of which are active during walking (thoracocoxopodite joint (TC) and coxo-basipodite joint (CB)) and the inactive mero-carpopodite (MC) joint). The TC joint is the most proximal of seven joints in a crayfish limb and moves in the rostro-caudal plane. The $\mathrm{CB}$ joint is the next distal joint and moves the limb in the dorso-ventral plane. The MC joint is the fifth-most proximal joint and normally moves the distal leg in the dorso-ventral plane. This joint is inactive to reduce the degrees of freedom allowed to distal leg 


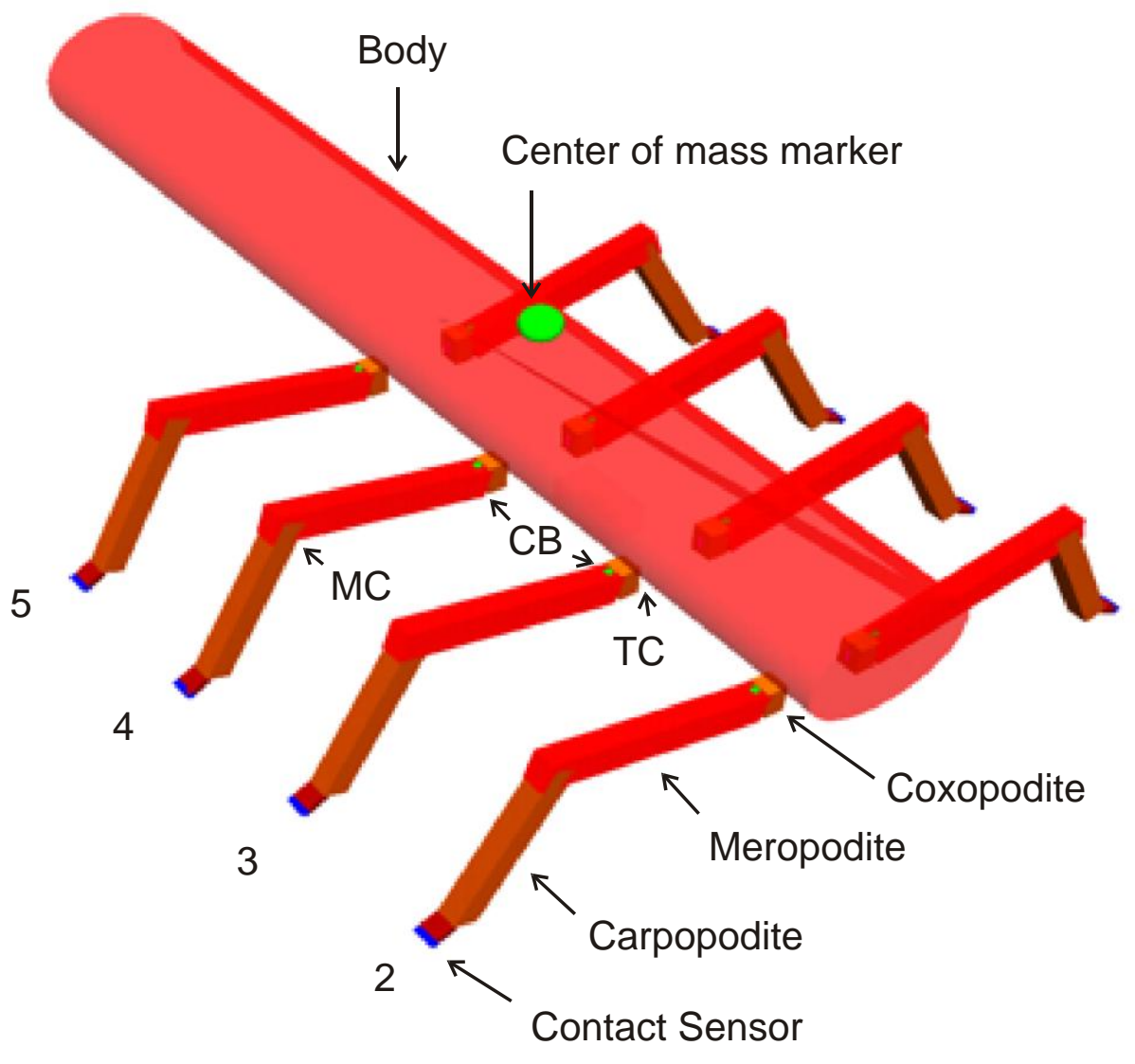

Figure 5.1. Body plan of virtual crayfish. The crayfish was built with a $15 \mathrm{~cm}$ body representative of a thorax and abdomen. Legs are numbered from 2 to 5 (anterior to posterior). The crayfish has eight identical legs, each with three segments (coxopodite, meropodite, carpopodite) and three joints (thoracocoxopodite (TC) joint, coxo-basiopodite (CB) joint, mero-carpopodite (MC) joint). 
movements and to simplify neural control of the limb. Therefore, the MC joint makes the distal leg function as a strut. Studies on lobsters have shown that the MC joint and distal leg can act as a strut during actual decapod walking (Ayers and Clarac, 1978).

Joint ranges of motion are also restricted. Crayfish, as well as most decapods, have ranges of motion at the joints that greatly exceed those observed during walking (Vídal-Gadéa et al., 2009). Range of motion at the $\mathrm{TC}$ joint is limited to $70-110^{\circ}$ around the null position of $90^{\circ}$. The primary reason for this is to prevent collisions between legs and the potential for adjacent legs to tangle, but $40^{\circ}$ is also a typical range of motion observed in TC joint movements of real crayfish during walking (Jamon and Clarac, 1997; Chapter 4). CB joints are limited to 5-25 from their original position of $15^{\circ}$. This, too, is a normal observed range of motion for this joint (Jamon and Clarac, 1997; Chapter 4). Limiting the CB joint in this manner also prevents inclusion of detailed mechanisms of postural control in the behavioral network.

\section{Behavioral plan / Neural circuitry}

Motor control is identical in all eight of the legs. Each leg is driven by two oscillators: one controls the TC joint, the other controls CB joint function. Work on stick insects has suggested that each leg joint is driven by its own oscillator (Büschges et al., 1995). The model oscillators are based on a half-center model and use reciprocal inhibition to produce rhythmic output (Graham Brown, 1914; Friesen, 1994). Cells making up the TC and CB oscillators have identical membrane and synaptic properties. Each half of each oscillator excites a motor neuron that provides input to one of the joints (Figure 5.2). The TC oscillator excites two motor neurons, a promotor which causes the leg to move rostrally, and a remotor which causes the leg to move caudally. The $\mathrm{CB}$ oscillator excites levator and depressor motor neurons which cause the leg to move up and down, respectively. Decay rates at the synapses and accommodation factors in the cells produce cycles with a period of approximately $2 \mathrm{~s}$, each half-center fires for half the period, or $\sim 1$ second. This period is similar in duration to the stepping cycle of an adult crayfish leg walking at a medium to slow pace (Chapter 4). 

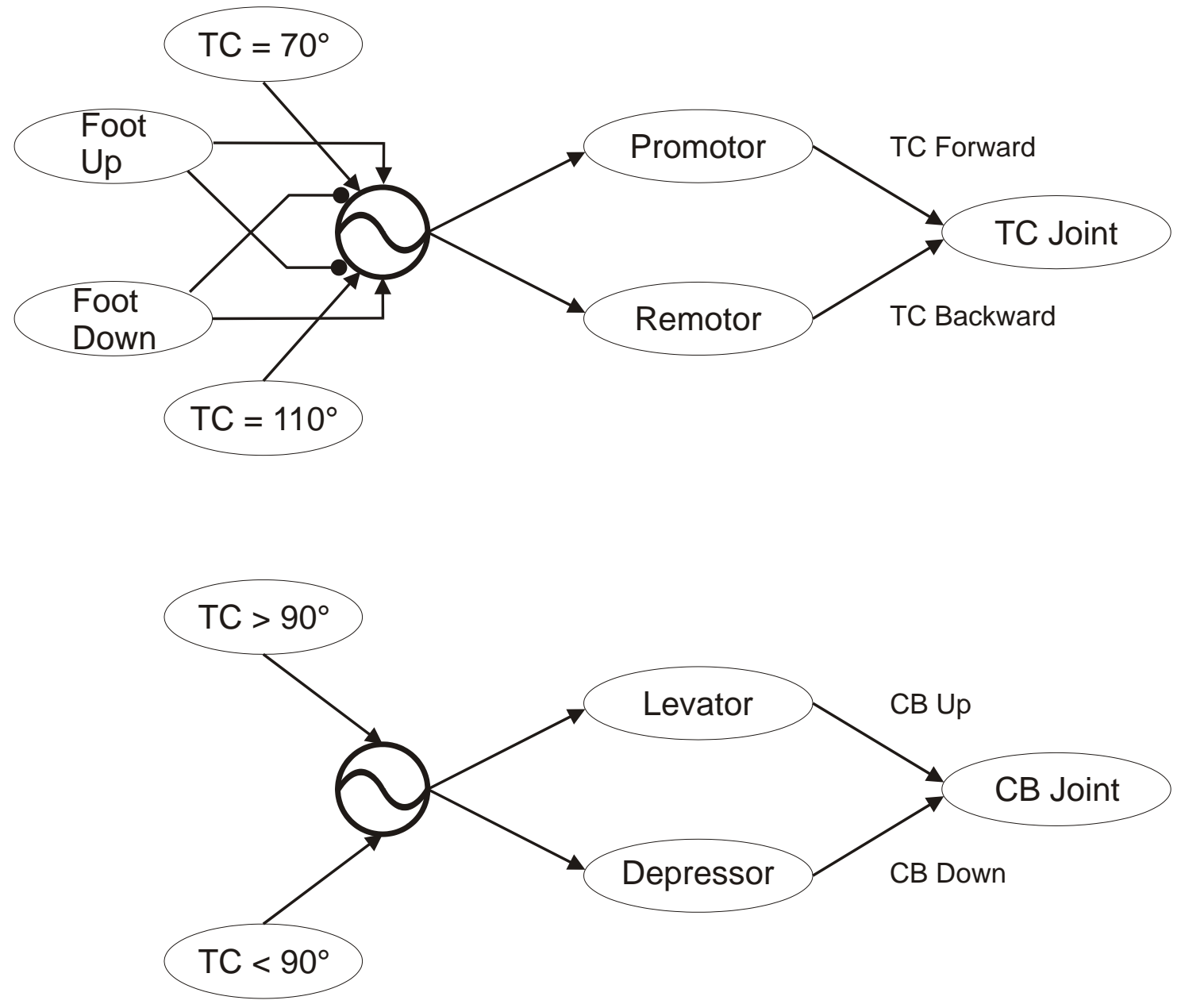

Figure 5.2. Wiring diagram of an individual leg. Each leg has two oscillators: one driving the TC joint and one driving the $\mathrm{CB}$ joint. The $\mathrm{CB}$ oscillator is coupled to the $\mathrm{TC}$ oscillator by position sensors on the TC joint. The TC oscillator receives feedback from position sensors in the TC joint. The TC oscillator also receives inputs from contact sensors on the end of the leg. 
The oscillators receive inputs from position sensors in the TC joint. In the crayfish, chordotonal organs located at the joints in the crayfish leg measure displacement of the leg segment distal to that joint (review by Cattaert and LeRay, 2001). Four sensory neurons representative of chordotonal organs were used at the model TC joint to measure position changes. Two of these neurons are excited when the TC joint reaches one of its extreme ends of range of motion. When the limb reaches its anterior extreme position (AEP) as determined by a $70^{\circ}$ displacement of the TC joint, a sensory neuron (named TCCO AEP2) is excited. This neuron provides an excitatory input to the half of the oscillator that drives the remotor neuron and moves the TC joint in the opposite direction. The model leg has a complimentary mechanism that fires the promotordriven half of the oscillator when the TC joint reaches its posterior extreme position (PEP) determined by a $110^{\circ}$ displacement of the joint. The remaining two chordotonal cells measure any displacement from $90^{\circ}$. One is excited by any anterior displacement; the other is excited when the leg displays posterior displacement. These two cells provide input to the $\mathrm{CB}$ oscillator and effectively link the two oscillators. If the TC is displaced towards the anterior, the TCCO AEP excites the depressor half of the CB oscillator. If the TC is displaced posteriorly, the TCCO PEP excites the levator causing the leg to lift. Activation of this circuit results in movement of the distal leg in an elliptical motion around the null position in the axial plane. The displacement is elongated in the rostro-caudal direction due to the larger TC range of motion. The kinematic output of the two model joints is based largely on kinematic analysis of the fourth leg of real animals (Jamon and Clarac, 1997; Chapters 2,4). The fourth leg was chosen because this is the leg nearest the center of mass in most walking conditions. It is also the most likely of the four legs to cross a $90^{\circ}$ orientation with the longitudinal body axis during real walking.

The model has two additional sensory cells that provide feedback to the TC oscillator. These sensory neurons are activated by contact sensors on the end of the leg (dactyl). The contact sensor sends an excitatory signal to a "Foot Down" neuron when contact with the substrate is made. The Foot Down neuron inhibits another cell named Foot Up. Therefore, one of these two cells is always firing during walking. The Foot Down neuron sends excitatory inputs to the remotor half of the TC oscillator and inhibitory inputs to the promotor half. The Foot Up neuron has the opposite role; it excites the promotor half and inhibits the remotor half of the TC 
oscillator. These sensory neurons are analogous to funnel canal organs (FCOs) in the dactyl of crustacean walking legs are excited by contact with the substrate (Müller and Clarac, 1990).

\section{Experimental procedure}

Simulations began with animals in the middle of a $100 \mathrm{~cm}^{2}$ area. The animals were allowed to walk until they reached the edge of the substrate. In every trial animals walked in a straight line. Trials usually lasted for 65-70s, but the first 10s of each trial was discarded. During the beginning of most trials, the model displayed erratic behavior as individual TC and CB leg oscillators established a rhythm.

\section{Treatment of data}

Interlimb coordination was quantified in the form of phase relationships between two legs. Phase values were calculated by determining the onset of a leg's step cycle relative to the cycle period of a reference leg (Chapter 2-4). Phase relationships were calculated for all legs relative to their ipsilateral and contralateral neighbors. For the amputation experiments, R5 and R3 were considered neighboring legs when R4 was removed. When both R3 and R4 were removed, R5 and R2 became ipsilateral neighbors.

Step variables were also quantified. Four variables were measured: mean periods (duration of a step cycle), mean swing ratios (swing duration / period), mean stance durations, and mean swing durations. All step variables were analyzed similar to the same data in the behavioral experiments (Chapters 2-4).

Gait diagrams were generated for four walking conditions: an eight-legged crayfish, amputation of leg R4, amputation of legs R3 and R4, and a four legged crayfish (legs L2-R2 and L5-R5). Gait diagrams were quantified using the same method presented in previous chapters 2-4. 


\section{Results}

\section{Walking behavior}

The first goal was to determine if the model construct could produce realistic interlimb coordination during walking. The model behavior was remarkably similar to actual crayfish. Coordination was more tightly distributed in posterior legs. Peak phase distributions showed that ipsilateral legs 3-4 were predominantly alternating while legs 4-5 were almost always alternating (Figure 5.3A). Contralateral legs 4 and 5 also showed predominantly alternating coordination but with a slightly wider, slightly offset, distribution than ipsilateral neighbors (Figure 5.3B). This is very similar to interlimb coordination in real crayfish (Chapters 2,4 ). The phase distributions suggest limb coordination in which legs 4 and 5 alternate both ipsilaterally and contralaterally. Most of the time leg 3 showed a similar coordination suggesting that the model animal uses an alternating tripod gait for the majority of its steps.

When inputs from the dactyl contact sensors were eliminated, ipsilateral coordination became extremely variable in the posterior legs but increased in the anterior legs (Figure 5.3C). The coordination between ipsilateral legs 2 and 3 did not show as tight a distribution as legs 4 and 5 when the sensors were intact. Contralateral legs also showed a change in behavior. Contralateral legs 5 showed no defined coordination after contact sensors were removed. However, contralateral legs 2 showed an increase in alternating coordination. Contralateral legs 4 also showed a preferred coordination. With no contact sensors, the phase distribution shifted towards a value of one implying a more in phase coordination.

The effects of contact sensors on step components were also investigated. With contact sensors, the model crayfish showed shorter cycle periods and shorter stance durations in the anterior legs (Figure 5.4A). Removing the contact sensors resulted in more uniformity across legs with respect to cycle and stance durations (Figure 5.4B). Feedback from the contact sensors to the oscillators can cause a change in the period of a step cycle. 


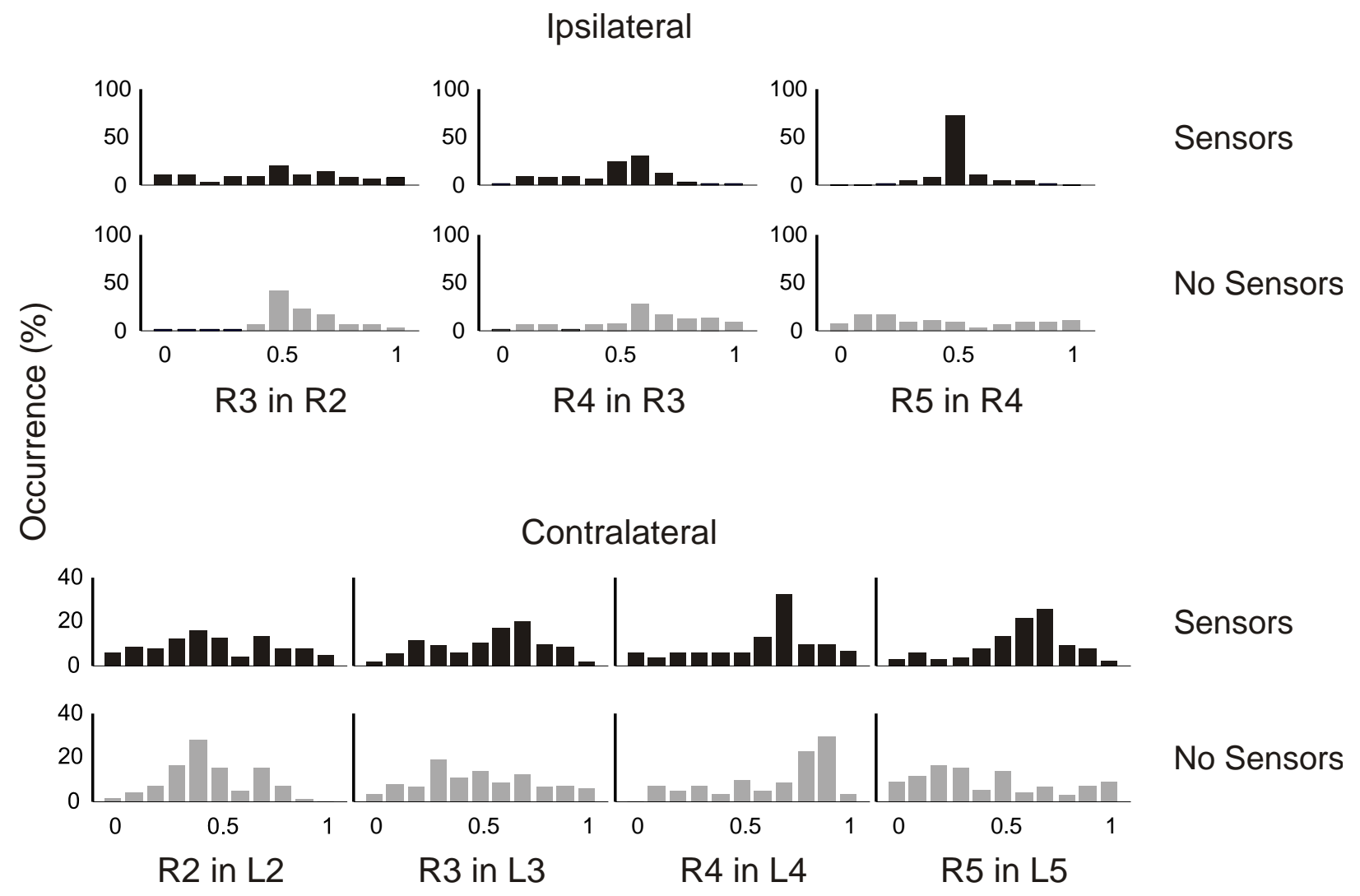

Figure 5.3. Phase relationships of model crayfish legs with functioning contact sensors (black) and without contact sensors (gray). Ipsilateral phase relationships show a high occurrence of alternating coordination between legs 4 and 5 when contact sensors are intact (top row). Without contact sensors, leg 4 and 5 coordination became more variable; however, anterior legs 2 and 3 showed more alternation (second row). Contralateral phase relationships are more variable in anterior legs with intact contact sensors (third row). Contralateral legs 2 alternated in the absence of contact feedback (bottom row). Leg 4 also showed a distinct trend in contralateral coordination that was shifted toward in phase. In both cases ipsilateral phase relationships showed higher occurrences (note difference in y-axis between the top two rows compared to the bottom two rows). 
A

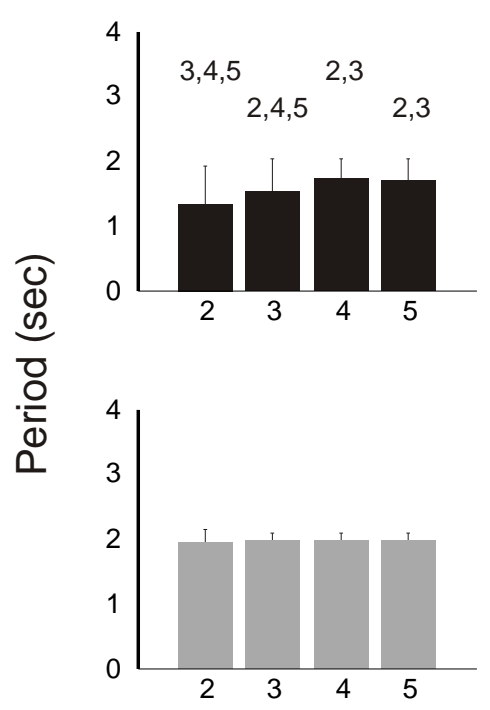

C

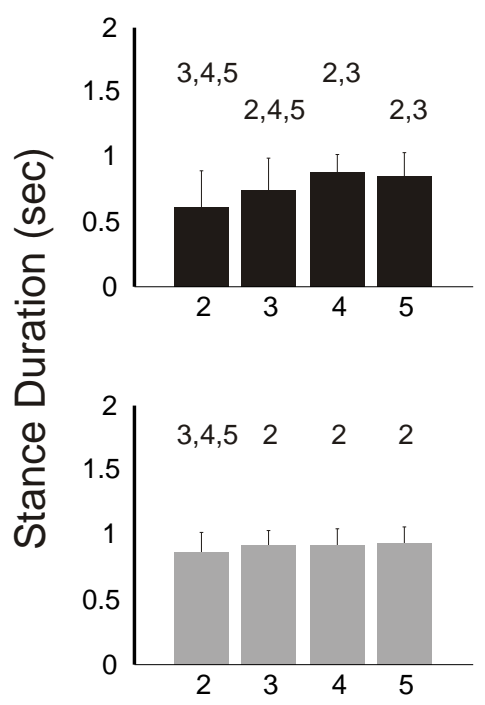

B

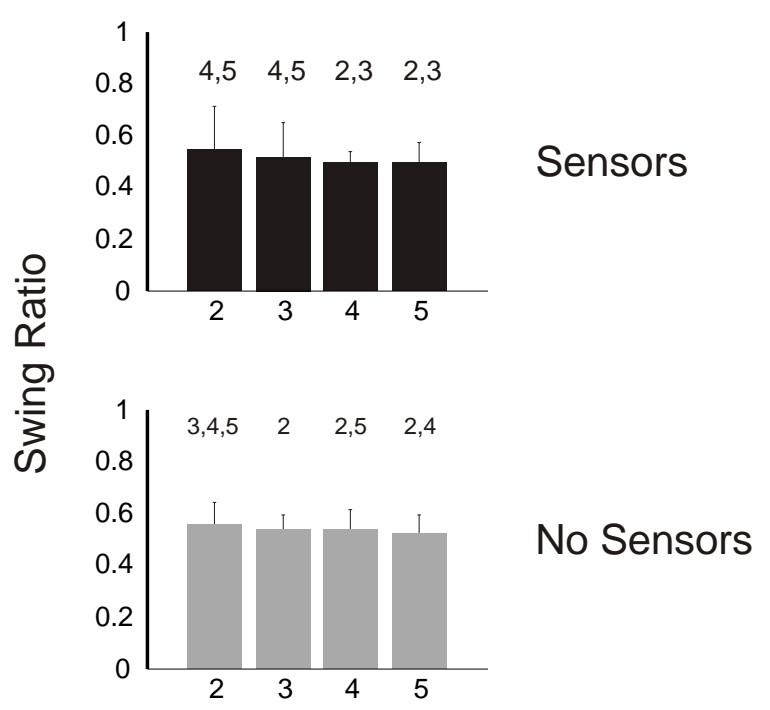

D

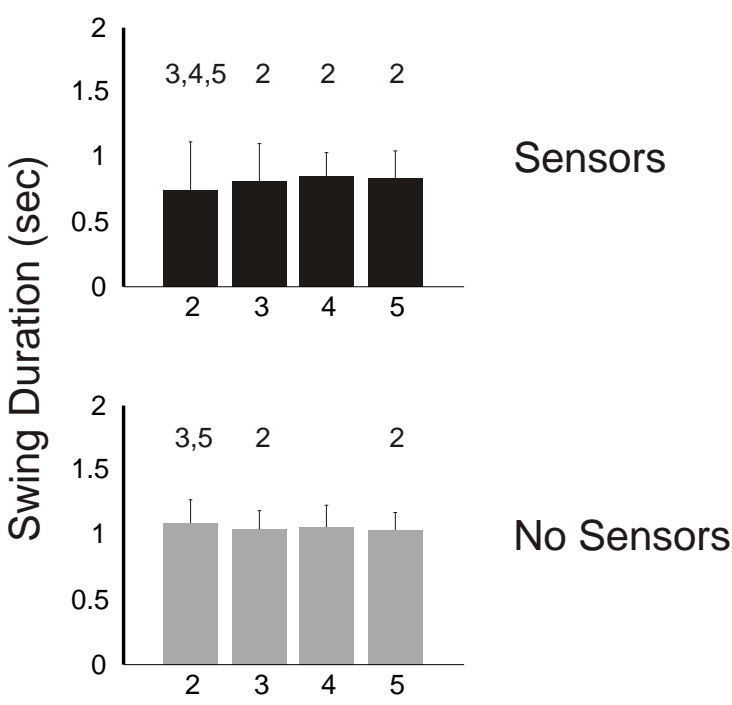

Leg

Figure 5.4. Step variables with (black bars) and without (gray bars) contact sensors. A) Cycle periods show differences between legs with contact sensors intact (numbers identify legs with a significant difference of $p<0.01$; one-way ANOVA). All legs cycle with the same period without contact sensors. Periods increase in each leg after contact sensors are removed (all legs showed significant differences without contact sensors, one-way ANOVA, $p<0.01$ ). B) Anterior legs 2 and 3 have longer swing ratios with contact sensors (Kruskal-Wallis nonparametric test, $p<0.01$ ). When contact sensors were removed limb behavior was uniform. C-D) Stance and swing durations showed similar trends as leg periods when sensors were functional (one-way ANOVA). After removal of sensors, leg 2 showed a change in function. 


\section{Center of mass influence on coordination}

The effect of changing the location of the center mass was tested next. The center of mass was placed in three locations: equidistant from legs 2 and 3 hinge points, then legs 3 and 4 , and finally legs 4 and 5 . Under all three conditions, the ipsilateral legs nearest the center of mass showed strong alternating coordination (Figure 5.5). A more anterior center of mass resulted in strong alternation between legs 2 and 3 as well as legs 3 and 4 (Figure 5.5A). Coordination between legs 4 and 5 was more variable as phase distributions appeared to be bimodal with small peaks in phase distribution at 0.5 and $0 / 1$, or out- and in-phase. Shifting the center of mass to a location between legs 3 and 4 (also the midpoint between all eight legs), caused alternating coordination in all ipsilateral legs (Figure 5.5B). The more posterior center of mass resulted in predominantly alternating coordination between ipsilateral legs 3 and 4 . However, this center of mass location resulted in phase values 0.5-0.6 for all steps comparing leg 4 to leg 5 (Figure 5.5C). Coordination between legs 2 and 3 became highly variable when the center of mass was farther away. The behavior of the model suggests that the closer legs are to the center of mass, the more likely they will alternate with their ipsilateral neighbors.

Contralateral coordination in all legs showed the same trends as ipsilateral coordination except with wider, more variable, distributions (Figure 5.6A-C). Alternation was more likely in contralateral legs nearer the center of mass. When the center of mass was in a medial location to all legs, coordination was variable across all legs.

\section{Center of mass influence on step variables}

Step variables were also affected by the location of the center of mass. The most obvious changes in behavior occurred in legs 2 and 5. An anterior center of mass resulted in mostly uniform behavior in the step components of legs 2-4. However, leg 5 showed distinct differences. Leg 5 showed shorter periods suggesting the leg cycled at a higher frequency than the other three legs (Figure 5.7A). Leg 5 also showed a higher swing ratio, meaning that for a given step this leg was off the substrate for a longer amount of time. This coincides with the observed mean stance and swing durations. Leg 5 showed much shorter stance durations but 


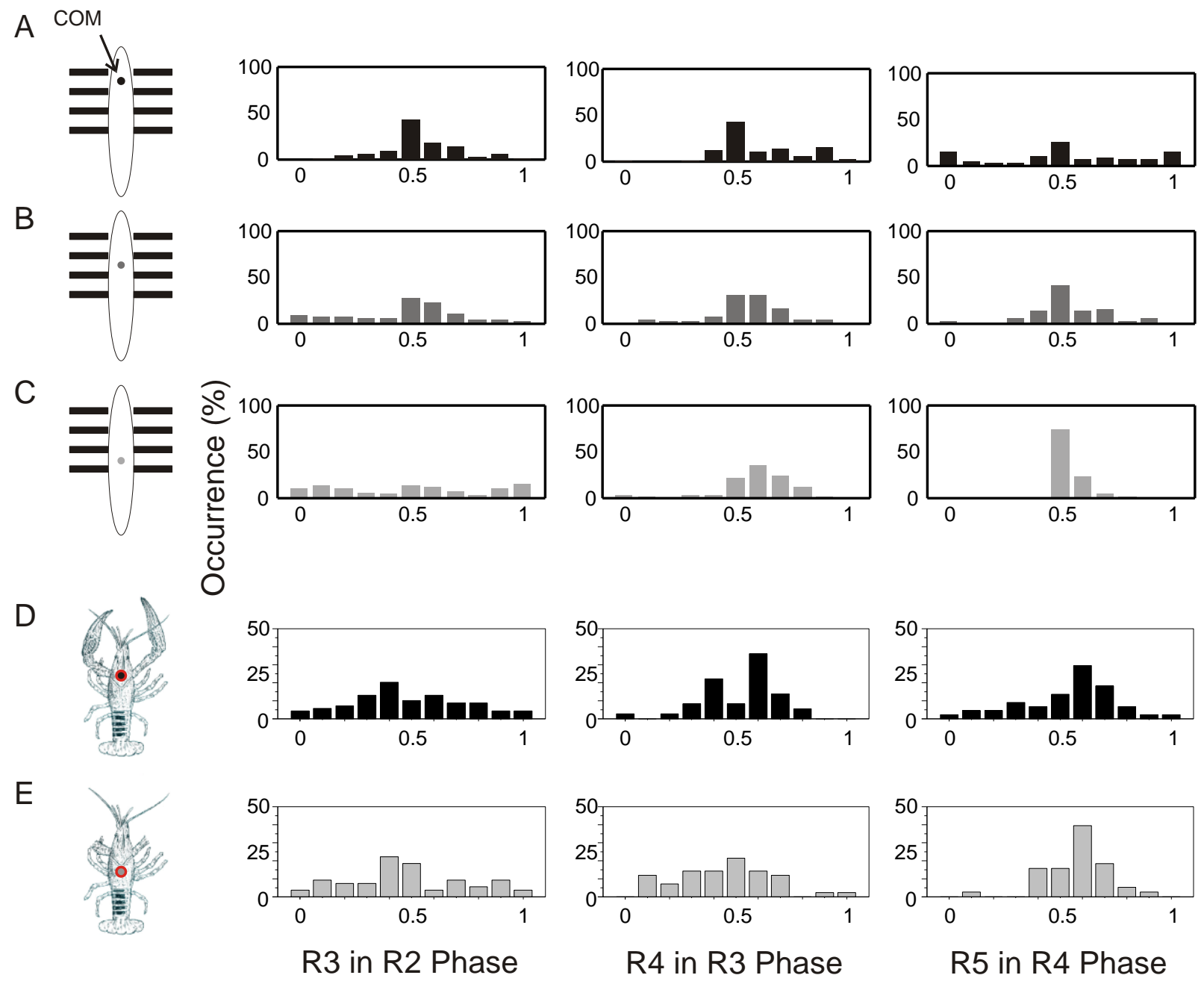

Figure 5.5. Ipsilateral phase relationships during simulated walking with three different COM locations (A-C) are compared to behavioral data from adult crayfish (D-E). Pictures on the left side of the figure represent location of center of mass relative to the animal's body. A) An anterior center of mass results in more occurrences of alternating coordination in anterior legs. B) A central center of mass shows alternation of all ipsilateral legs. C) Posterior center of mass gives predominantly alternating coordination in legs 4 and 5. D-E) Kinematic analysis of adult crayfish show similar responses in limb coordination with a shift in center of mass. 


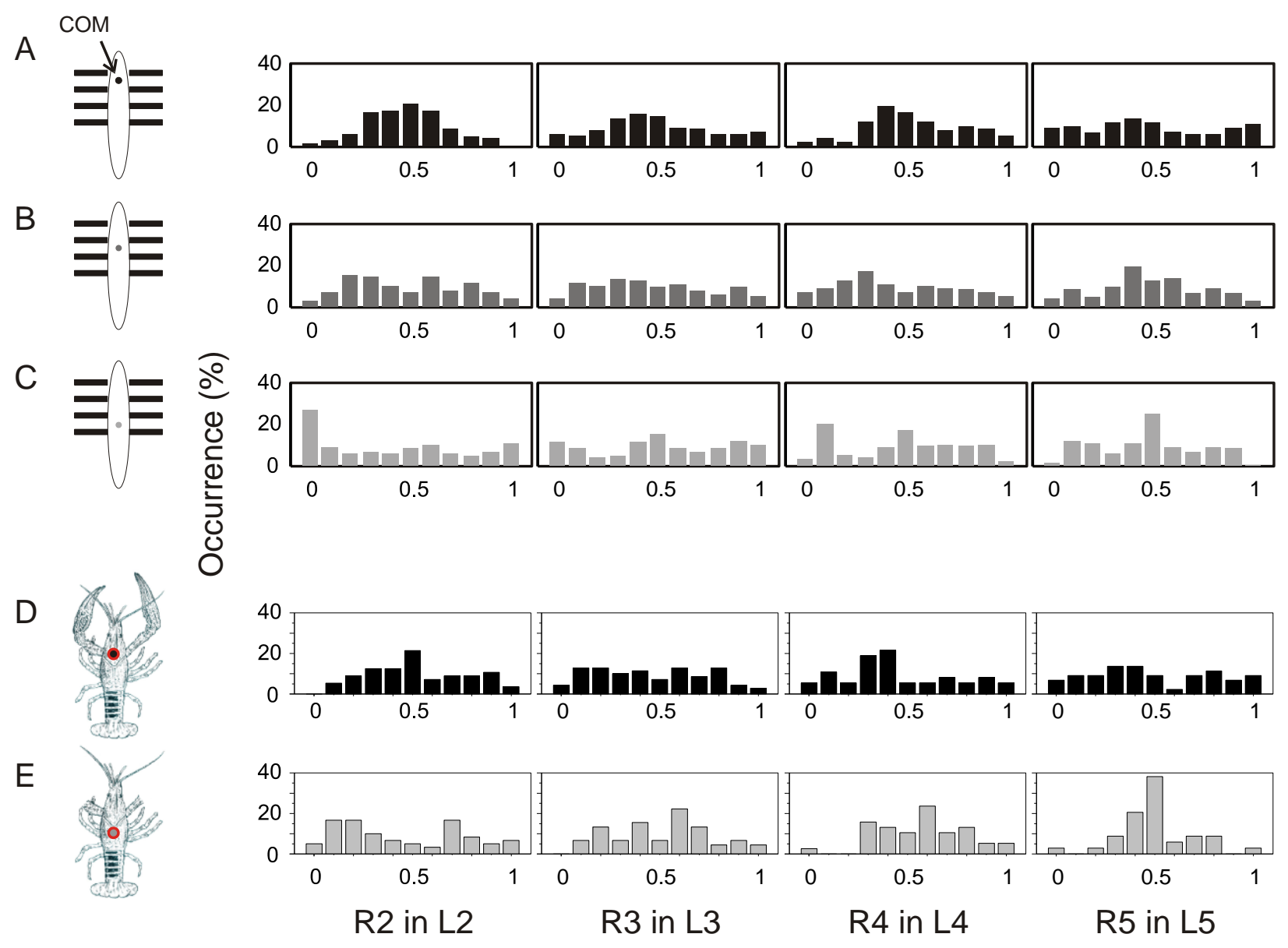

Figure 5.6. Contralateral phase distributions cluster at or near 0.5 in those legs closer to the COM. Model phase distributions (A-C) are compared with data from adult crayfish with different centers of mass (D - E). 

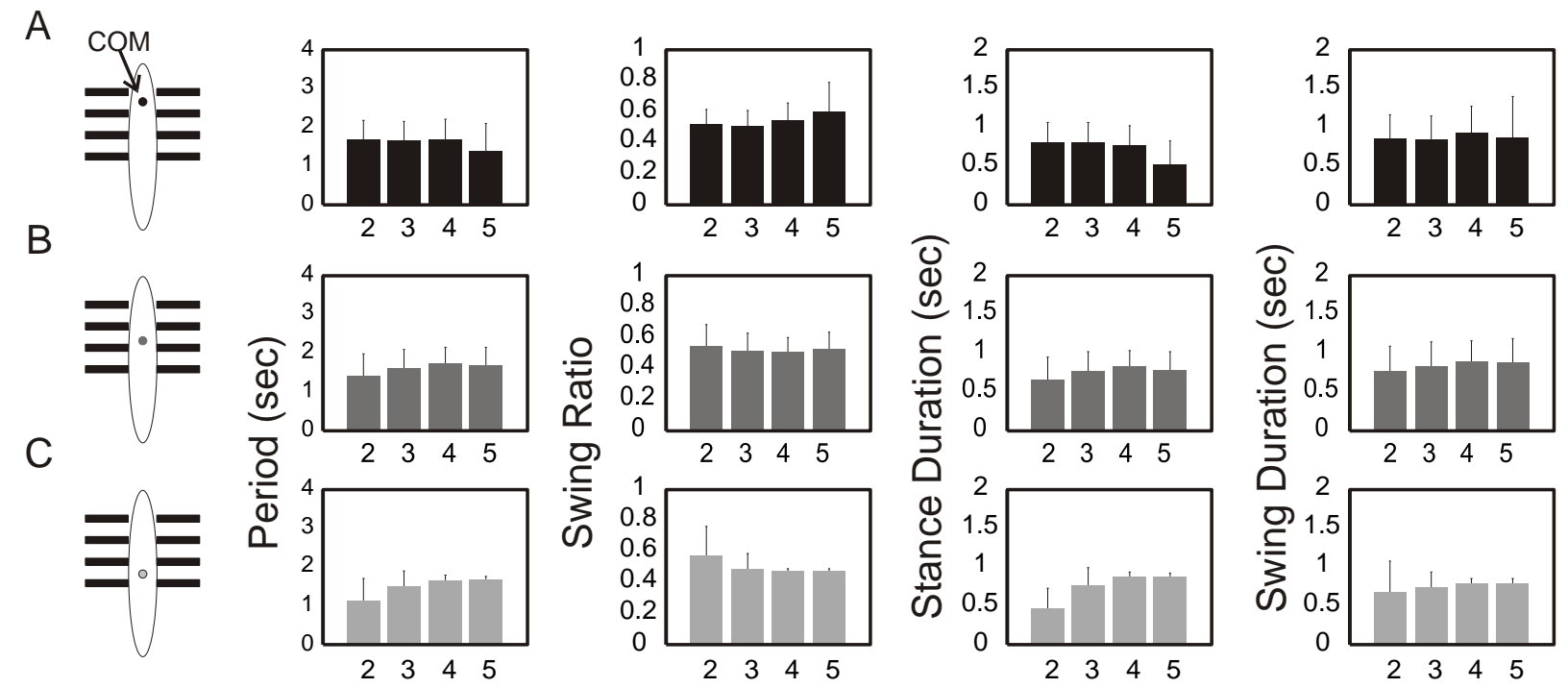

D
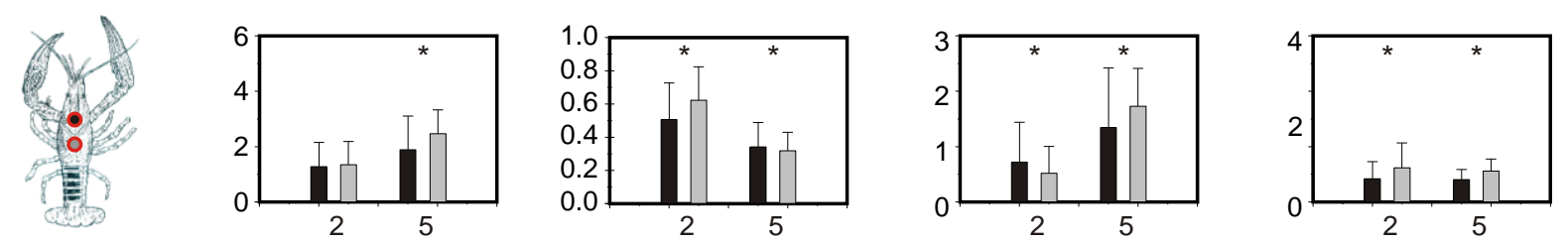

Leg

Figure 5.7. Step variables change with center of mass location. A) An anterior center of mass produces a unique function in leg 5. B) When the center of mass is centrally located, leg 5 acts more like legs 3 and 4 while leg 2 starts to show differences. C) A posterior center of mass location results in a larger change in leg 2. D) Changes in the model are similar to those observed in adult crayfish with the exception of swing duration (data taken from Figure 4.2). 
maintained similar swing durations as the other legs. When the center of mass was placed between legs 3 and 4, behavior changed in legs 2 and 5. Under this condition, leg 5 behavior became more similar to legs 3 and 4 (Figure 5.7B). Leg 2 showed changes that resembled those observed in leg 5 when the center of mass was more anterior. Cycle periods and stance durations in leg 2 were shorter with a more posterior center of mass. A further shift in the center of mass between legs 4 and 5 amplified these changes in behavior. With the center of mass even farther from leg 2, there was a larger decrease in its step period and stance duration. There was also a noticeable increase in the leg 2 swing ratio, much like that of leg 5 in the anterior center of mass condition (Figure 5.7C). Therefore, it appears that legs nearest the center of mass show stepping properties in which cycle periods and stance durations are longer while swing ratios become shorter.

\section{Perturbations to the model / Amputation study}

For the amputation experiments the center of mass was placed directly over the insertion points for leg 4, same as the control experiments. When leg R4 is removed legs R3 and R5, which show variable coordination under normal conditions, show very high occurrences of alternation (Figure 5.8). Also, the coordination between legs R3 and R2 displayed a preferred stepping pattern. On the left side of the body, L4 and L5 maintained a phase relationship similar to that seen prior to R4 removal.

When legs R3 and R4 were remove, the phase distribution between legs R2 and R5 was at or very near 0.5 for $100 \%$ of the trials. Again, legs L5 and L4 still showed a predominantly alternating behavior suggesting coordinating influences between these legs were unchanged. Changes in gait were limited to the coordination of neighboring ipsilateral legs.

Stepping patterns of the model crayfish under intact and amputation conditions show changes in limb coordination as limb number is reduced (Figure 5.9). Model gaits are remarkably similar to juvenile gaits under similar conditions (Figure 5.9B). 

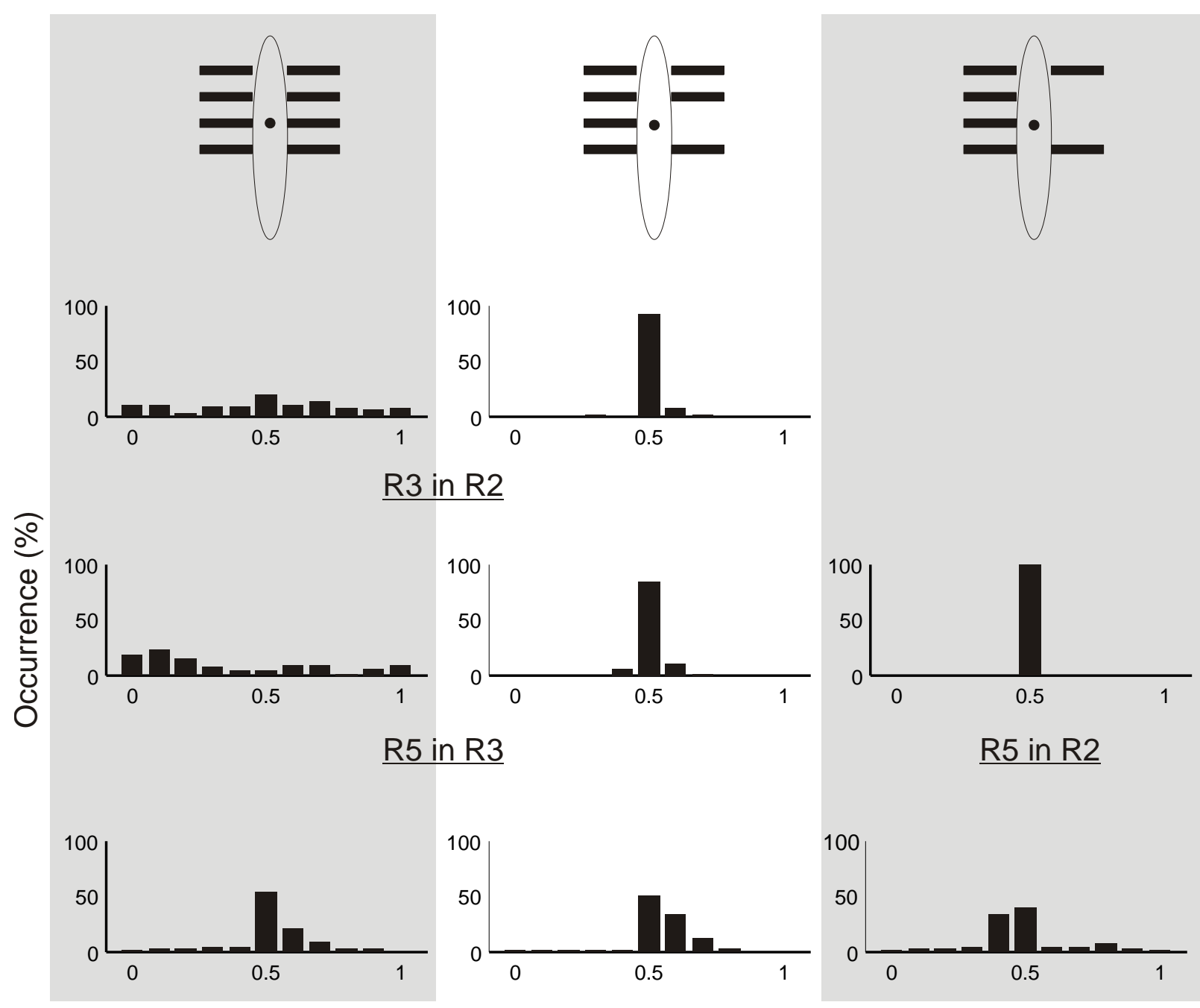

$\mathrm{R} 5$ in R3

$\underline{\mathrm{R} 5 \text { in } \mathrm{R} 2}$
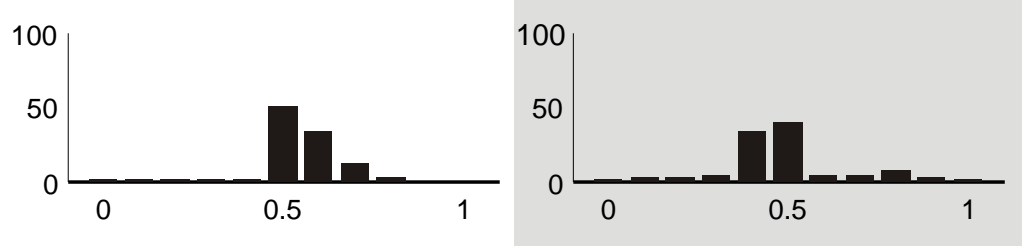

\section{L5 in L4}

Figure 5.8. Phase relationships changed when one, then two legs, were removed from the model. Intact neighboring legs always maintained a strong alternating coordination during whole animal experiments (A), with leg R4 removed (B), and with legs R3 and R4 removed (C). The data also show that the fewer ipsilateral legs, the stronger the coordination between neighboring legs on the side of limb amputation. 


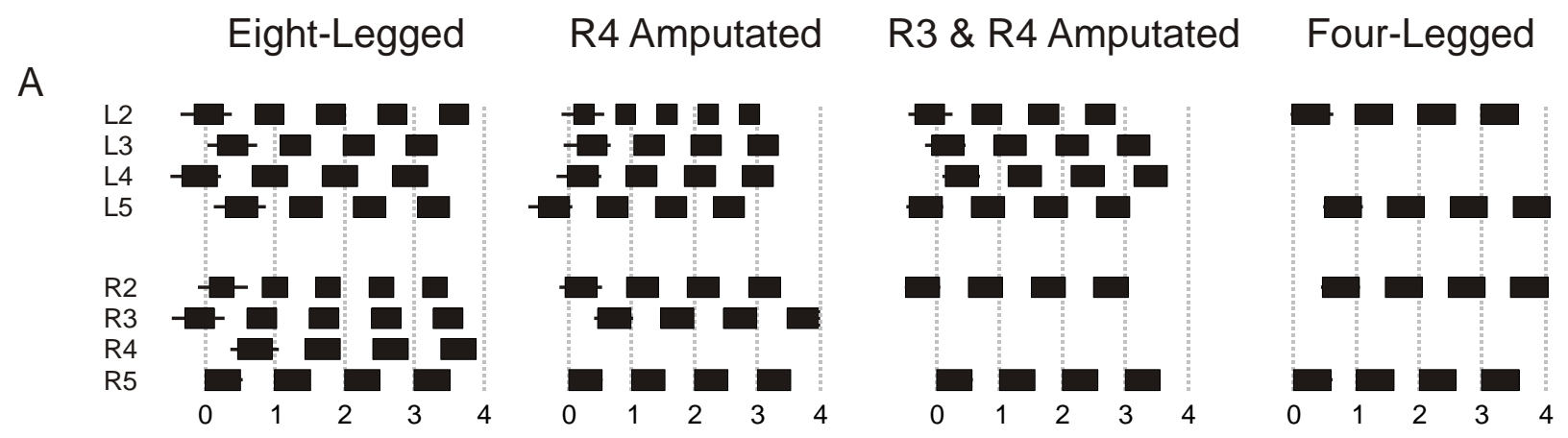

B
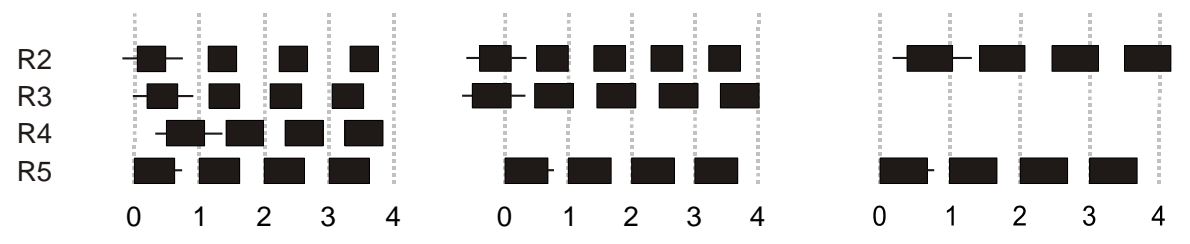

Leg R5 Step Cycle

Figure 5.9. A) Model gaits of an eight-, seven-, six-, and four-legged crayfish. B) Stepping patterns of right legs of juvenile crayfish under similar conditions (presented in Chapter 3). Boxes indicate stance; open areas between boxes show swing in that leg. Error bars to the left side of stance boxes represent standard deviation of stance onset in that leg relative to leg R5 stance onset. Error bars on the right side of the stance boxes represent standard deviation of that leg's swing onset relative to leg R5 stance onset. (Model data are the average of one trial: eight-legged, $\mathrm{n}=32 ; \mathrm{R} 4$ amputated, $\mathrm{n}=32 ; \mathrm{R} 3$ and $\mathrm{R} 4$ amputated, $n=29$; four legs, $n=35$. Real animals: eight-legged, $n=140 ; R 4$ amputated, $n=102 ; R 3$ and $\mathrm{R} 4$ amputated, $n=84$. Sample sizes are the number of steps of the R5 reference leg.) 


\section{Four-legged crayfish}

A four-legged animal has fewer potential limb combinations that can produce support and, therefore, fewer combinations of gaits that can hold the animal up while also producing forward movement. Therefore, simulations were run to determine if the model could successfully walk as a quadruped. The model was successful and produced a very stereotyped gait in which diagonal legs moved in phase with each other and alternated with the opposite diagonal pair (Figure 5.9). The four-legged walker did stumble on occasion. During stumbling bouts, the model would temporarily lose the necessary interlimb coordination required for forward walking. However, after a few small steps, the model always regained coordination and resumed walking.

\section{Discussion}

The crayfish model shows that multi-legged walkers do not need the nervous system to provide coordinating mechanisms between legs to produce realistic behavior. Gravitational forces influence stereotyped movements of individual legs resulting in behavior that is observed in real crayfish. This is achieved by mechanical coupling through the environment. The leg oscillators produce an elliptical trajectory at the tip of the leg, or dactyl. At any given point in the dactyl trajectory the leg has the potential to occupy stance or swing. Stance and swing phases are dependent upon the geometrical orientation between the body and the ground (Figure 5.10). If the body maintains a parallel orientation with the ground, as it does with a centrally-positioned center of mass, all legs should contact the ground at the same point in their trajectories. A posterior shift in the center of mass causes a postural pitch in the animal due to the rigidity of the body. This shift results in a new orientation between body and ground that effectively changes the contact points within leg trajectories. Lowering the posterior end of the animal means potential dactyl contact points in posterior legs are more dorsal in the trajectory path.

Conversely, anterior legs have less possible trajectory space to contact the ground. This explains why stance and swing durations change with center of mass location in the model. Actual crayfish show similar trends in legs based on proximity to the center of mass (Chapter 4). This could be a physical mechanism that changes the state of the leg without the need for a control 

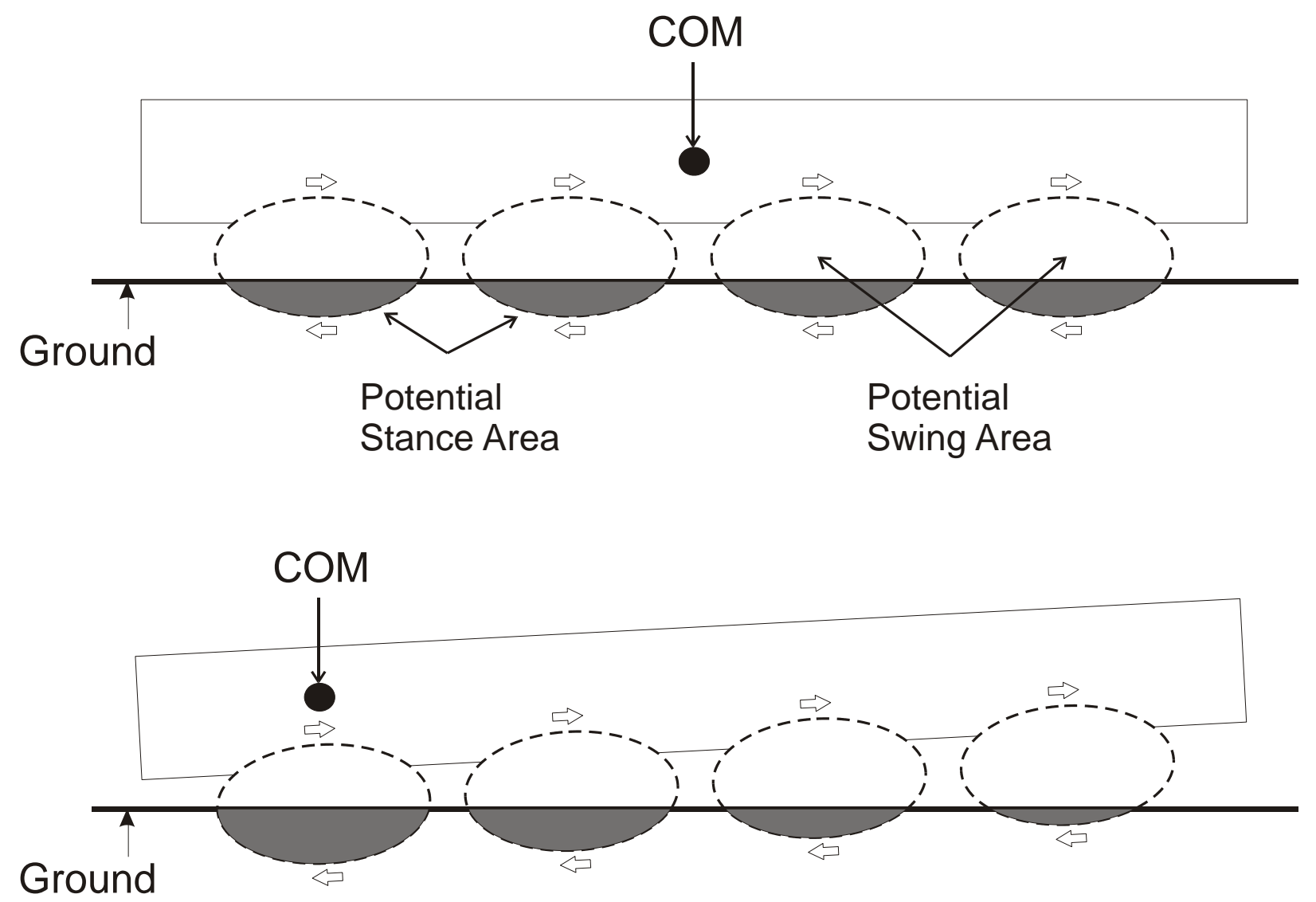

Figure 5.10. Idealized diagram of four identical dactyl trajectories (dashed ellipses) relative to a rigid body and the ground. When the center of mass (COM) is located near the center of the body, load distribution is uniformly distributed across the legs (top diagram). Step variables, stance and swing, are potentially the same in all legs. If the center of mass is shifted caudally, there is a change in posture of the animal that causes a posterior pitch (bottom diagram). This results in a new load distribution across legs. The posterior legs now have a greater area within the dactyl trajectory that is associated with stance. Conversely, the anterior legs show greater potential swing areas. This is the physical constraint that produces variability in the step variables of the model. 
mechanism in the limb (Figure 5.4B, D). Without contact feedback, periods remain the same across all legs.

Introducing contact feedback to the leg oscillators has two effects. First, contact inputs amplify the differences in stance and swing durations between legs. The state of the leg (Foot Up or Foot Down) has a feed-forward effect on that leg. If a leg is in the stance phase, the Foot Down neuron excites the remotor half of the oscillator and inhibits the promotor half. This feedforward mechanism can be stopped by either reaching the maximum TC joint displacement (in which case the $\mathrm{CB}$ oscillator elevates the leg off the substrate if there is sufficient postural support elsewhere), or by being lifted off the substrate by the physical mechanism described above. The second effect of contact feedback is changes to the cycle frequency of the oscillators. When contact sensors were added to the legs, all periods were significantly shorter. This occurs because transitions between stance and swing immediately switch the activity of the oscillators. With no feedback the oscillators produced dactyl trajectories without discrimination of the state of that leg. The trajectories can be shortened with contact feedback if a state transition occurs before the leg reaches the limits of its spatial parameters. Periods in legs farthest from the center of mass are significantly shorter than all other periods. This is a result of the physical mechanism acting on the structural system and the feed-forward mechanism in the neural circuitry. These leg trajectories have small potential stance areas because of posture relative to the ground (Figure 5.10). When these legs do make contact it is usually for a relatively short time. The feed-forward mechanism at these sometimes rapid step transitions results in greatly reduced trajectories. The aesthetic result appears remarkably similar to "double steps" in actual animals (Chapter 2).

Because there are no coordinating programs in the CNS of the model, limb coordination is an emergent property of collective limb and body dynamics relative to the ground and environmental influences. Figure 5.10 shows body to ground orientation under static conditions. Animals, real and virtual, experience postural pitch, yaw, and roll displacements as they move. The crayfish model does not have elastic elements. Therefore, all postural displacements are more representative of a rigid-bodied system (Cavagna et al., 19977; Blickhan and Full, 1987) than in other models using elastic components (spring-loaded models, Raibert et al., 1986; Schmitt and Holmes, 2000ab). The result of a rigid-bodied model means that the body will fall 
towards areas of instability at the end of the stance trajectory of a given leg. Muscles and tendons (elastic elements) can produce kinetic energy at the end of a stance trajectory that counteracts the fall of the body (Full and Koditschek, 1999). Following body and center of mass trajectories of the rigid system over several steps shows the mechanism by which limb coordination emerges. A center of mass located within a base of support of three or more legs is statically stable (Figure 5.11) (Full et al., 2002). When only two legs are contacting the substrate, the center of mass is considered statically unstable and will fall in a direction based on the sum of the forces acting on the body (gravity plus the forces produced by the legs in contact with the ground). This scenario will produce a change in body posture resulting in a new spatial relationship between leg trajectories and the ground. As long as the body to ground angle does not reach parallel, the legs directly anterior and adjacent to the center of mass will have the next largest potential stance area in their trajectory. This increases the probability that these legs will acquire a stereotyped coordination with those legs currently in contact with the substrate. Walking is forward-directed and mass is distributed primarily in the rostro-caudal plane (VídalGadéa et al., 2009). Therefore, the magnitude of force acting on the center of mass is primarily anterior-directed. This causes ipsilateral adjacent legs nearest the center of mass to acquire strict alternating coordination. Orthogonal displacements, yaw and roll, are less severe but still large enough to influence contralateral coordination. This agrees with findings in real crayfish and lobsters that show ipsilateral coordination is more stereotyped than contralateral coordination (Chapters 2-4; Cruse and Müller, 1986; Müller and Cruse, 1991a). This same mechanism sufficiently explains how legs acquire different coordinations after amputation of leg(s) as well as that seen in four-legged animals. The four-legged model occasionally lost coordination because there are larger displacements of the center of mass in all planes and fewer legs to compensate for a temporary loss of support.

Previous animal-inspired, multi-legged (6 or more) models have used designs in which leg controllers have inputs to and from adjacent legs (Beer, 1990; Cruse et al., 2007). In vitro and in situ studies support the idea that intersegmental neural signaling may be present in ipsilateral legs (Sillar et al., 1987; Borgmann et al., 2007). In many of these cases, rhythmic output in ipsilateral hemiganglia is in-phase or very near in-phase. Sillar et al. (1987) also found that the ipsilateral synchrony showed no preferred coordination between contralateral hemiganglia in the nerve cord. Therefore, neural connectedness in the 

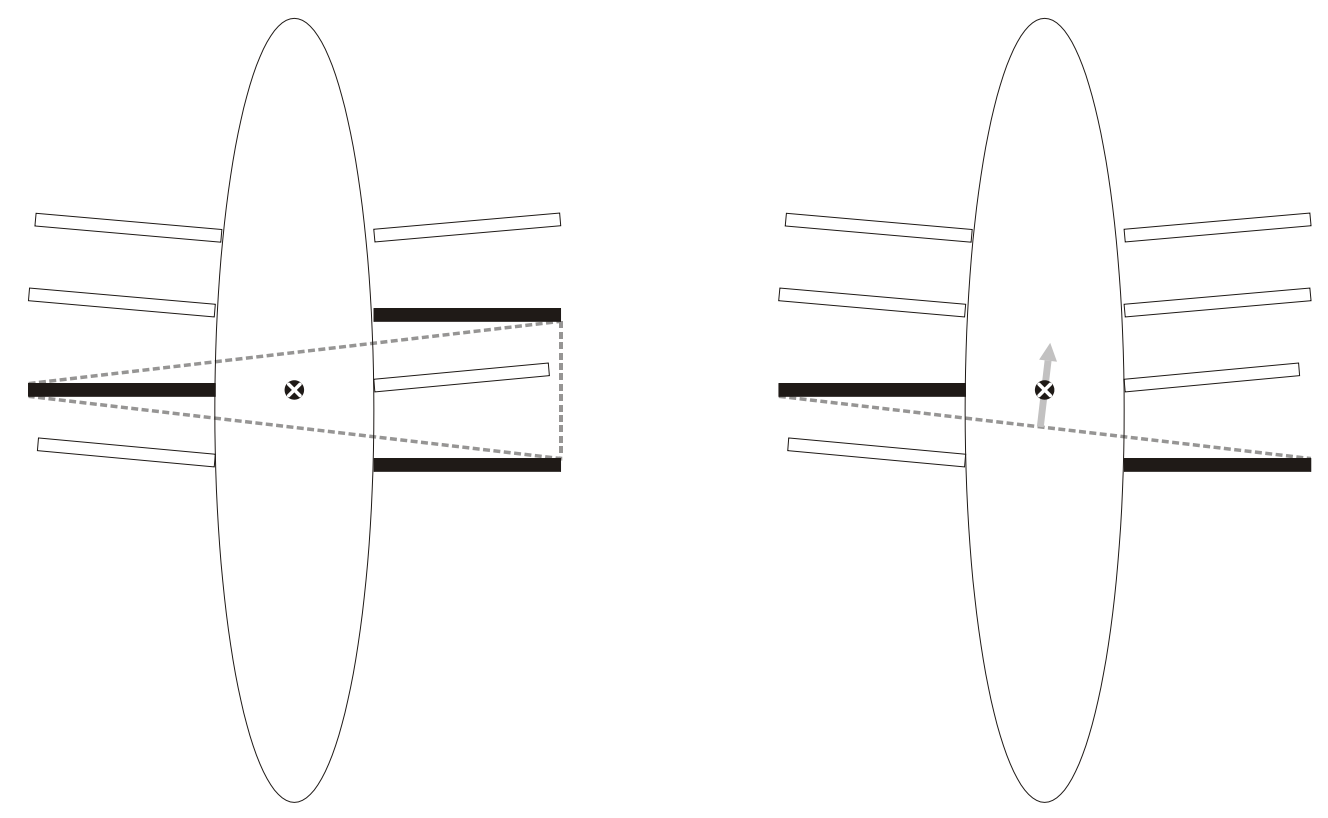

Figure 5.11. A walking system is statically stable when the center of mass (left, black circle with cross) lies within a base of support. The figure on the left shows three legs in contact with the substrate (black). The center of mass is within the base of support (dashed line). The figure on the right shows a statically unstable scenario. In this situation the center of mass will fall in the direction of the arrow. 
walking nervous system may be between ipsilateral legs only, but this coordination does not look like actual walking behavior. The present study shows that the interlimb neural circuitry is not necessary to produce realistic walking and that mechanically coupling the legs through the environment by way of the body and center of mass is sufficient. A recent study in cockroaches showed that load receptors in an isolated leg fire when an adjacent leg has relieved it of load (Zill et al., 2009). This sudden decrease in the leg's load is correlated with swing onset of that leg. This would suggest that cockroach legs do not need to be coupled neurally because the load receptor measures the mechanical coupling and can potentially keep a leg programmatically isolated while the animal shows a highly coordinated gait. However, the model presented here shows that these gaits can be achieved without load feedback. Therefore, it may be possible that more complex neural systems used by real animals are redundant mechanisms that have evolved due to the environmental constraints shown in the model.

In summary, the crayfish model shows realistic walking behavior can be the result of a relationship between organism and environment that is more dependent upon physical influence than previously realized. A multi-legged organism does not need highly integrated controllers to produce stable walking behavior. The behavior of individual legs, their step components, interlimb coordination, stability, and walking behavior itself arise as emergent properties of a simple neuro-mechanical system under collective interactions with the environment. AnimatLab

and its real-world Vortex ${ }^{\mathrm{TM}}$ physics engine have allowed this hypothesis to be tested in three dimensions under environmental conditions that are not easily controllable in actual behavioral experiments.

\section{References}

Ayers, J. L. and Clarac, F. (1978). Neuromuscular strategies underlying different behavioral acts in a multifunctional crustacean leg joint. J. comp. Physiol. 128, 81-94

Barnes, W. J. P. (1977). Proprioceptive influences on motor output during walking in the crayfish. J. Comp. Phsiol. (Paris) 73, 543-564 
Bässler, U and Büschges, A. (1998). Pattern generation for stick insect walking movements multi-sensory control of a locomotor program. Brain Res. Rev. 27, 65-88

Beer, R.D. (1990). Intelligence as adaptive behavior: an experiment in computational neuroethology. Chandrasekaran, B. (ed.). Academic Press, Inc., San Diego

Blickhan, R. and Full, R.J. (1987). Locomotion energetic of the ghost crab. II. Mechanics of the center of mass during walking and running. J. exp. Biol. 130, 155-174

Borgmann, A., Scharstein, H., and Büschges, A. (2007). Intersegmental coordination: Influence of a single walking leg on the neighboring segments in the stick insect walking system. $J$. Neurophysiol. 98, 1685-1696

Büschges, A., Schmitz, J., and Bässler, U. (1995). Rhythmic patterns in the thoracic nerve cord of the stick insect induced by pilocarpine. J. exp. Biol. 198, 435-456

Cattaert, D. and Le Ray, D. (2001). Adaptive motor control in crayfish. Progress in Neurobiology. 63, 199-240

Cavagna, G.A., Heglund, N.C., and Taylor, C.R. (1977). Mechanical work in terrestrial locomotion: two basic mechanisms for minimizing energy expenditure. Am. J. Physiol. 233, R243-R261

Chasserat, C. and Clarac, F. (1980). Interlimb coordinating factors during driven walking in crustacean. J. comp. Physiol. 139, 293-306

Chrachri and Clarac, F. (1990). Fictive locomotion in the fourth thoracic ganglion of the crayfish, Procambarus clarkii. J. Neurosci. 10(3), 707-719

Clarac, F. and Chasserat, C. (1979). Experimental modifications of interlimb coordination during locomotion of a Crustacea. Neurosci. Lett. 12, 271-276

Clarac, F. and Barnes, W. J. P. (1985). Peripheral influences on the coordination of the legs during walking in decapods crustaceans. In: Coordination of motor behavior. Bush, B.M.H. and Clarac, F. (eds.). Cambridge University Press, Cambridge. pp 249-269 
Clarac, F. and Chasserat, C. (1983). Quantitative analysis of walking in a decapods crustacean, the rock lobster Jasus lalandii. I. Comparative study of free and driven walking. J. exp. Biol. $107,189-217$

Cofer, D.W., Reid, J., Zhu, Y., Cymbalyuk, G., Heitler, W.J. and Edwards, D.H. (2006).

AnimatLab: A physics based 3D graphics environment for behavioral neurobiology research. Computational Neuroscience, Edinburgh, UK, 2006

Cruse, H. (1976). The function of the legs in the free walking stick insect, Carausius morosus. J. comp. Physiol. 112, 235-262

Cruse, H. (1983). The influence of load and leg amputation upon coordination in walking crustaceans: A model calculation. Biol. Cybern. 49, 119-125

Cruse, H. (1990). What mechanisms coordinate leg movement in walking arthropods? Trends Neurosci. 13, 15-21

Cruse, H. and Müller, U. (1986). Two coupling mechanisms which determine the coordination of ipsilateral legs in the walking crayfish. J. exp. Biol. 121, 349-369

Cruse, H., Bartling, C., Dean, J., Kindermann, T., Schmitz, J. and Schumm, M. (2000). A simple neural network for the control of a six-legged walking system. Proc. 1993 IEEE/RSJ Int. Conf. Intell. Robot. Syst. 1, 545-552

Cruse, H., Dean, J., Dürr, V., Kindermann, T., Schmitz, J. and Schumm, M. (2002). A decentralized, biologically based network for autonomous control of (Hexapod) walking. Auton. Robots pp. 383-400

Cruse, H., Dürr, V. and Schmitz, J. (2007). Insect walking is based on a decentralized architecture revealing a simple robust controller. Phil. Trans. R. Soc. A 365, 221-250

Delcomyn. F. (1980). Neural basis of rhythmic behavior in animals. Science 210, 492-498

Friesen, W.O. (1984). Reciprocal inhibition: a mechanism underlying oscillatory animal movements. Neurosci and Biobehav Rev 18:547-553 
Full, R.J. and Koditschek, D.E. (1999). Templates and anchors: neuromechanical hypotheses of legged locomotion on land. J. exp. Biol. 202, 3325-3332

Full, R.J., Kubow, T., Schmitt, J., Holmes, P. and Koditschek, D. (2002). Quantifying dynamic stability and maneuverability in legged locomotion. Integ. And Comp. Biol. 42, 149-157

Goulding, M. (2009). Circuits controlling vertebrate locomotion: moving in a new direction. Nature Reviews Neuroscience. 10, 507-518

Graham Brown, T. (1914). On the nature of the fundamental activity of the nervous centres; together with an analysis of the conditioning of rhythmic activity in progression, and a theory of the evolution of function in the nervous system. J. Physiol. 48, 18-46

Grillner, S., Wallén, P., Brodin, L., and Lasner, A. (1991). Neuronal network generating locomotor behavior in lamprey. Апnи. Rev. Neurosci. 14, 169-200

Grote, J. R. (1981). The effect of load on locomotion in crayfish. J. exp. Biol. 92, 277-288

Hill, A.A.V., Masino, M.A., and Calabrese, R.L. (2003). Intersegmental coordination of rhythmic motor patterns. J. Neurophysiol. 90, 531-538

Hughes, G.M. (1952). The co-ordination of insect movements. I. The walking movements of insects. J. exp. Biol. 29, 267-284

Jamon, M. and Clarac, F. (1995). Locomotor patterns in freely walking crayfish (Procambarus clarkii). J. exp. Biol. 198, 683-700

Jamon, M. and Clarac, F. (1997). Variability of leg kinematics in free-walking crayfish, Procambarus clarkii, and related inter-joint coordination. J. exp. Biol. 200, 1201-1213

Jones, S.R. and Koppell, N. (2006). Local network parameters can affect inter-network phase lags in central pattern generators. J. Math Biol. 52, 115-140

Kato, M. (1990). Chronically isolated lumbar spinal cord generates locomotor activities in the ipsilateral hindlimb of the cat. Neurosci. Res. 9, 22-34 
Kindermann, T. (2002). Behavior and adaptability of a six-legged walking system with highly distributed control. Adapt. Behav. 9, 16-41

Kjaerulff, O. and Kiehn, O. (1997). Crossed rhythmic synaptic input to motoneurons during selective activation of the contralateral spinal locomotor network. J. Neurosci. 17, 9433-9447

Kubow, T.M. and Full, R.J. (1999). The role of the mechanical system in control: a hypothesis of self-stabilization in hexapedal runners. Phil. Trans. R. Soc. Lond. B. 354, 849-862

Ludwar, B. C., Göritz, M. L. and Schmidt, J. (2005). Intersegmental coordination of walking movements in stick insects. J. Neurophysiol. 93, 1255-1265

Müller, U. and Clarac, F. (1990). Dactyl sensory influences on rock lobster locomotion. I. Intrasegmental and intersegmental leg reflexes during standing and walking. J. exp. Biol. 148, 89-112

Müller, U. and Cruse, H. (1991a). The contralateral coordination of walking legs in the crayfish Astacus leptodactylus. I. Experimental results. Biol. Cybern. 64, 429-436

Namba, H. and Mulloney, B. (1999). Coordination of limb movements: three types of intersegmental interneurons in the swimmeret system, and their responses to changes in excitation. J. Neurophysiol. 81, 2437-2450

Pond, C.M. (1975). The role of the 'walking legs' in aquatic and terrestrial locomotion of the crayfish Austropotambius pallipes (Lereboullet). J. exp. Biol. 62, 447-454

Raibert, M.H., Chepponis, M., and Brown Jr., H.B. (1986). Running on four legs as thought they were one. IEEE J. Rob. Auto. RA-2, 70-82

Schmitt, J. and Holmes, P. (2000a). Mechanical models for insect locomotion: dynamics and stability in the horizontal plane. I. Theory. Biol. Cybern. 83, 501-515

Schmitt, J. and Holmes, P. (2000a). Mechanical models for insect locomotion: dynamics and stability in the horizontal plane. II. Application. Biol. Cybern. 83, 517-527 
Sillar, K.T., Skorupski, P., Elson, R.C., Bush, B.M.H. (1986). Two identified afferent neurons entrain a central locomotor rhythm generator. Nature. 323, 440-443

Sillar, K. T., Clarac, F. and Bush, B. M. H. (1987). Intersegmental coordination of central neural oscillators for rhythmic movements of the walking legs in crayfish. Pasifastacus leniusculus. J. exp. Biol. 131, 245-264

Tschuluun, N., Hall, W.M., Mulloney, B. (2001). Limb movements during locomotion: Tests of a model of an intersegmental coordinating circuit. J. Neurosci. 21(19), 7859-7869

Wendler, G. (1966). The co-ordination of walking movements in arthropods. Symp Soc Exp Biol. 20, 229-249

Wilson, D. M. (1966). Insect walking. Annu Rev Entomol. 11,103-151

Vídal-Gadéa, A., Rinehart, M.D., Belanger, J.H. (2009). Skeletal adaptations for forwards and sideways walking in three species of decapod crustaceans. Arthropod Struct. Dev. 37, 95-108

Zill, S.N., Keller, B.R., Duke, E.R. (2009). Sensory signals of unloading in one leg follow stance onset in another leg: Transfer of load and emergent coordination in cockroach walking. $J$. Neurophsiol. 101, 2297-2304 
Chapter VI: Summary and Conclusions 


\section{Behavioral Experiments}

Theoretical results show that over a lifetime a crayfish may experience a significant change in the relative environment in which it lives and walks. Reynolds numbers for extremely small and large crayfish suggest a change of four orders of magnitude, indicating that juveniles exist in a more viscous relative environment, which, in turn, could influence walking kinematics. Juveniles and adults can and do walk differently. However, at very slow walking speeds, adult walking kinematics looks more like juvenile walking behavior. When tested, the actual difference in Reynolds number of the animals here was only two orders of magnitude, which, according to a rule of thumb, does not represent a significant difference in Reynolds number (Alexander, 2003). Therefore, the relative environments of the adult and juvenile crayfish may not be so different as to significantly influence walking kinematics, initially predicted.

Although juveniles and adults have very similar body morphologies, it could be possible that the portion of the juvenile nervous system controlling walking has not completely developed. Amputating selected limbs reduces sensory feedback to the leg central pattern generators. The resulting rhythmic activity of the leg stumps resembles the endogenous component of the nervous walking system (Clarac and Chasserat, 1979; Sillar et al., 1987). Juvenile crayfish showed no differences with adults in the function of individual leg oscillators or coordination between adjacent oscillators (Clarac and Barnes, 1985; Sillar et al., 1987; Chrachri and Clarac, 1990; Chapter 3). The synchronous activity of adjacent oscillators is also the same in other arthropods (Borgmann et al., 2007), suggesting many animals may share a similar endogenous component in the walking system. Intact legs in juveniles also adopted a new coordination in response to these perturbations. This change in behavior also occurs in adult crayfish and lobsters (Clarac, 1978; Clarac and Barnes, 1985; Grote, 1981). These findings effectively rule out developmental changes in the nervous system as a cause for differences in walking kinematics between juvenile and adult crayfish and many species of arthropods.

Crayfish limb and body morphologies scale proportionally with the exception of the chelae. Adult chelae are approximately twice as large as juveniles, relative to body length. The consequence of larger adult chelae is a more anterior location of the center of mass. A shift in center of mass during crayfish ontogeny suggests that adults and juveniles have relatively 
different load distributions on their legs. This appears to be the main cause for the observed differences in walking kinematics. In experiments where centers of mass were shifted so that adults had a load distribution similar to juveniles, many aspects of adult walking kinematics more resembled that of juveniles. The same was true for the posterior legs of juveniles where they were loaded to induce similar center of mass locations as adults. These observations further support the findings in the treadmill experiments that walking motor systems are functionally the same in juveniles and adults. Changes in limb function and coordination that result with changes in center of mass locations reveal that discrete portions of the walking motor system (i.e., the limbs) show flexible behavior depending on proximity to the center of mass. Therefore, based on the results of all of these behavioral experiments, it appears that the walking nervous system is the same no matter the age of the crayfish; moreover, control mechanisms for each leg are probably similar. Small shifts in center of mass produce changes in behavior that are localized to individual legs, indicating that these control mechanisms may be isolated to individual legs and separate from other legs. Because interlimb coordination can vary based on relative load distributions, strict coordinating mechanisms between legs are not necessary for control of walking.

\section{Model experiments}

The conclusions drawn from the behavioral experiments raised several questions about how motor control is coordinated. To date, scientists do not have the resources or knowledge necessary to test these hypotheses in biological systems. This is because little is known about the circuitry of the walking nervous system and physiologists do not know where precisely to look for answers. Modeling the walking nervous system in a virtual crayfish that walks under real-world conditions allowed elements of the walking nervous system to be tested that are not readily accessible in real animals. A model crayfish with no neural signaling between legs showed that walking is not only possible, but looks very similar to realistic walking behavior. The model also responded to limb perturbations in exactly the same way as real animals. Furthermore, a four-legged system can also produce successful, realistic behavior. Model behavior suggests that components of walking (i.e., limb coordination, posture control, and 
stability) arise from collective limb behavior and are dependent on the morphological form of the animal.

Motor control from the model walking nervous system only directs individual legs to produce spatial trajectories independent of the function of the other legs. The only perceptual link to the environment comes from feedback that influences oscillator activity based on whether or not a leg is in contact with the substrate. Coordination emerges because legs are indirectly and mechanically linked through the ground and the animal's body. Interlimb coordination, and ultimately walking stability, is a function of the spatial orientation of the animal's body relative to the ground and the trajectory of its dactyls. Leg number and center of mass can produce new walking behaviors. Therefore, the principles that allow the model to walk effectively are not isolated to crayfish and can be applied to all walking systems.

\section{Biomechanical principles}

Other walking models have taken more generalized approaches to elucidate basic biomechanical principles that affect locomotion (Kubow and Full, 1999; Schmitt and Holmes, 2000ab). In these experiments, degrees of freedom for body and limb movements are reduced. AnimatLab and its real-world physics engine, Vortex ${ }^{\mathrm{TM}}$, have made it possible to test a simplified neuromechanical model with real-world degrees of freedom.

In the crayfish model, legs nearest the center of mass produce coordination that results in the most support of the animal's weight due to their relative spatial orientation. Because there are no elastic elements present in the biomechanics of the model, the trajectory of the center of mass can be conceptualized as an inverted pendulum (Figure 6.1) (Cavagna et al., 1977; Blickhan and Full, 1987). Without elastic elements in the legs, the trajectory of the center of mass falls after moving anterior to the vertical plane formed with the leg if no anterior supports are available. When elastic elements are added (i.e., muscles), the trajectory of the center of mass should gain height with extension of the muscle due to the kinetics (spring-loaded inverted pendulum (SLIP), Raibert et al., 1986). Without passive compression of the legs, the center of mass constantly falls at the end of a step and is caught by subsequent steps. In the SLIP model, potential energy is 


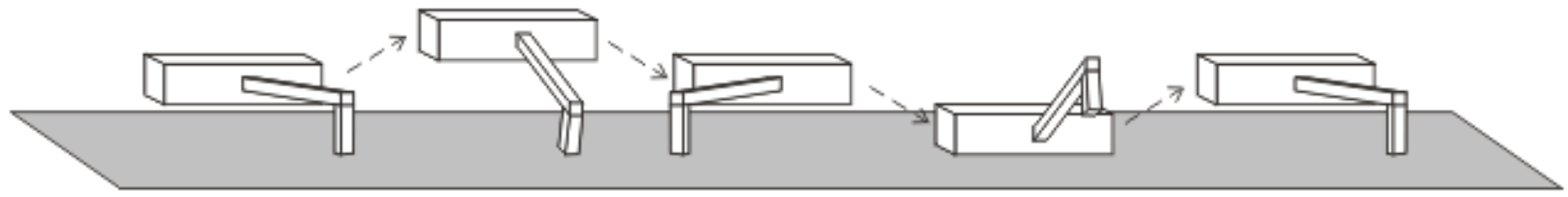

Figure 6.1. The trajectory of the body and its center of mass is idealized as an inverted pendulum. 
gained and stored in the spring in the first half of the step and is used as kinetic energy is used in the second half (Full and Koditschek, 1999). It may be possible that the neuromuscular system in its current form (more similar to the SLIP model) evolved in response to the biomechanical principles acting on the inverted pendulum. It is not difficult to imagine that mechanically coupled ipsilateral legs influenced the evolution of a walking nervous system that reinforces the coordination dictated by the environment.

The lateral leg spring model (LLS) provides a second template with which to compare the crayfish model. The LLS model includes body displacements in the horizontal (yaw) plane. Schmitt and Holmes (2000ab) designed a single-legged model based on cockroach walking in which legs were reduced to one. They incorporated a state-dependent mechanism in which the leg changed sides upon transition from stance to swing. In their model, realistic orientation and heading were achieved without neural controllers when the leg was placed posterior to the center of mass. Neural connectivity in contralateral legs is not necessary for producing and maintaining forward movement because yaw displacement in this body morphology is not as destabilizing as pitch displacement. Excessive lateral movements may produce erratic movement, but excessive pitch can result in falling. This may explain why ipsilateral coupling is stronger than contralateral coupling in the real and virtual animals (Müller and Cruse, 1991ab; Chapter 4). Therefore, if ipsilateral limbs are sufficiently coordinated, there will always be an available contralateral leg to limit yaw movements in a legged system with four or more legs. The crayfish model demonstrates this because phase relationships correlate between leg pairs (Figure 6.2). The crayfish model shows high occurrences of alternating contralateral coordination in legs posterior to the center of mass. This suggests that contralateral coupling is also a result of mechanical linkage, but fewer occurrences of alternating coordination suggest it is not as critical in maintaining forward walking because ipsilateral coordination is sufficient.

An example of emergent stability in a walking nervous system has been demonstrated here. The crayfish model shows how legs driven by isolated CPGs can find a coordination that provides walking stability. This is achieved by moments of static instability. During periods in which only one or two legs are on the ground, gravitational forces summed with multi-dimensional forces exerted by leg(s) on the substrate push the center of mass in the direction of instability. (This can be conceptualized by thinking of a three-legged stool that has one of the legs removed. 


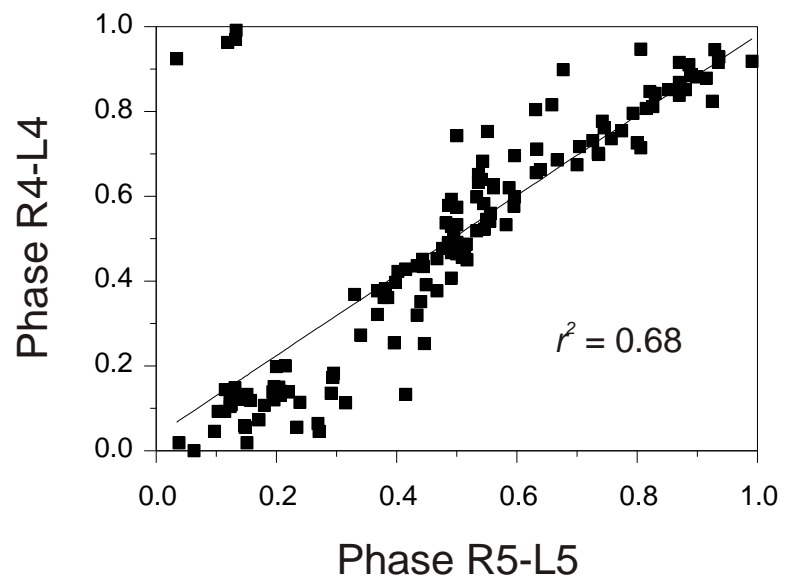

Figure 6.2. The model displayed strong correlation between contralateral phase values of subsequent steps in neighboring legs when the center of mass was located between them (Adjusted r-squared $=0.68$ ). 
The stool will fall in the direction of the missing leg.) The morphological design of the animal and the oscillatory behavior of the limbs insure that the falling body will be caught by another leg. This appears to be the underlying principle in actual crayfish locomotion because model and animal gaits respond in the same way to changes in load distribution.

\section{Coordination of crayfish movements}

Intersegmental coordination between oscillators in locomotor systems is an area of great interest with the most informative work coming from swimming systems, including crayfish (see reviews by Skinner and Mulloney, 1998; Hill et al., 2003). In these systems, segmental oscillators driving locomotion are usually strongly coupled to adjacent oscillators. Commissural neurons often provide contralateral coupling (Gillner et al., 1995) while other cells have been shown to carry inputs to adjacent ipsilateral ganglia and beyond (review by Goulding, 2009). Invertebrate walking systems have not been shown to contain this same level of complexity in neural connectedness. Interneurons in the crayfish swimmeret system can maintain rhythmic control in non-adjacent ganglia when the middle ganglion has been inhibited (Tschuluun et al., 2001). Studies on crayfish, lobsters, and stick insect locomotion have shown that these walking systems have ascending or descending patterns of leg activation from caudal to rostral ganglia, or vice versa (Clarac and Barnes, 1985; Cruse, 1990). It is attractive to think that this is the result of intersegmental neural coupling similar to that in the swimmeret system (Namba and Mulloney, 1999; Tschuluun et al., 2001). However, activation patterns may be an emergent property that results from local mechanical couplings between legs. The crayfish model shows the same activation patterns with stronger influences around the center of mass. Furthermore, the model demonstrates that legs nearest the center of mass have correlating contralateral coordination (Figure 6.2). This shows that contralateral coordination may be passed between legs, most likely as an indirect result of strong ipsilateral coupling. If this activity persisted between more anterior legs, the result would appear as an anterior-directed metachronal wave. These findings reflect those found in freely walking adult crayfish where two unique gaits were identified by correlation between leg 3 and 4 contralateral phase relationships (Jamon and Clarac, 1995). Leg 3 contralateral phase values correlated with leg 4 values of the preceding step. The model 
showed similar behavior between legs 4 and 5 with a posterior center of mass. This suggests that an intersegmental network is not necessary because the wave of activity is also a consequence of biomechanical influences. Furthermore, the required motor coordination between newly adjacent oscillators is different in the swimmerets and requires more flexibility during walking (especially if the animal loses a leg). Mechanically coupling the oscillators through the environment would ensure rhythmic coordination that produces successful walking behavior that can adapt to these types of perturbations.

\section{Control mechanisms: Neural vs. Mechanical, Slow vs. Fast}

There are currently two schools of thought concerning potential control mechanisms in slow and fast walking arthropods. It is assumed that slow walking animals, such as the stick insect, require control mechanisms that are primarily rooted in the nervous system, while fast walkers, such as the cockroach, use rapid mechanical reflexes to sustain locomotion (Cruse et al., 2007; Full and Koditschek, 1999). Results from the crayfish model suggest that slow walking animals do not need high levels of nervous control. Leg oscillators in the model cycle at a rate that produces a slow walking velocity, $1 \mathrm{~cm} / \mathrm{s}$, yet it achieves a realistic, stable gait with very little sensory feedback. Previous models with lower degrees of freedom that were able to produce successful walking without neural coupling were modeled after fast walkers, usually cockroaches (Schmitt and Holmes, 2000ab). Some slow-walking models have used artificial neural networks to control numerous components of walking (Kindermann et al., 2002; Cruse et al., 2000; Cruse et al., 2002). In these models, stance and swing transitions, as well as posture control, are all programmed into the networks at some level. Limb coordination is more loosely controlled but still relies heavily on programmed coordinating mechanisms based on limb function of multiple legs. These models are highly adaptive with robust behavioral output but the levels of control are just as highly complex. A simple neuromechanical system like the one in the crayfish model shows that stance-swing transitions, posture, limb coordination, and walking stability do not need to be programmed or computed by the nervous system; instead, they are direct consequences of the collective organism-environment interactions. Stance-swing transitions are directly affected by the body orientation relative to the substrate. Height control is affected by 
limb coordination because there is less pitch displacement when legs near the center of mass alternate. Therefore, walking stability is a result of a dynamic equilibrium between these interrelated walking parameters. Therefore, slow walkers do not need high levels of neural control and walking nervous systems likely share fundamental control components regardless of walking speed. However, limb kinematics in the crayfish model are restricted and the behavior is limited to forward walking. Therefore, more experiments are required to determine if uncoupled intersegmental oscillators can prove successful during other behaviors under different environmental conditions (i.e., turning behavior and walking over uneven terrain) where additional sensory feedback may be thought necessary.

Kubow and Full (1999) showed fast walkers could be self-stabilizing in the horizontal plane. The model crayfish showed self-stabilization at slow speeds and even accomplished this with only four legs. Part of the reason for this is the cyclical trajectories of the legs. As long as all legs are not cycling at the exact same phase, one leg proves effective to displace the center of mass enough so that the spatial orientation of another leg can continue its cycle without being in contact with the substrate. If all legs cycle in phase with one another, then the theoretical behavior of the crayfish would be rhythmic lifting and dropping of the body with the legs never leaving the substrate. The slightest phase offset would be enough to push the phase relationship of the legs toward alternating. Once a leg is allowed to move in non-zero phase with any other leg, the system is driven toward walking coordination. The observed limb coordinations are inevitable because once any leg is not in-phase the posture of the animal is affected. Therefore, height control of the animal is continuously adjusted until the most productive stepping rhythm is established. In every simulation, the model animal is self-stabilizing even when all legs are precisely in phase or fail to acquire a walking rhythm. In other words, it appears that slow walkers can use mechanical reflexes to stabilize themselves.

A second, closely-related debate is whether control systems are governed by oscillating networks or state-dependent systems. Fast-walkers are usually regarded as having oscillating networks with more rigidly controlled coordinating mechanisms (Beer, 1990; Full and Koditschek, 1999). In these systems, walking behavior is turned on and the animal moves according to the controller. Any necessary corrections to environmental perturbations are made "on the fly" by mechanical reflexes within elastic elements in connective tissues (Dudek and Full, 2006; 
Sponberg and Full, 2008). One group predicts that these systems respond too quickly to be controlled by reflexes in the CNS (Dudek and Full, 2006). Conversely, slow walkers are thought to use state-dependent systems for complex levels of control that have time to be mediated by the nervous system (Cruse et al., 2007). I argue that there is biological evidence of both properties within each system. Swimming and flying systems that function at higher frequencies than fastwalkers have bistable neurons in the CNS that produce plateau potentials representative of statedependent responses (lamprey, Wallén and Grillner, 1987; locust flight, Ramirez and Pearson, 1991ab). There is also evidence of plateau potentials in the cockroach walking system (Hancox and Pitman, 1991). In the non-walking systems, the bistable neurons are part of a larger oscillatory network that produces very rapid movement while adhering to the principles of a half center oscillator (Hancox and Pitman, 1993). Plateau potentials have been recorded in slow walking crayfish also (Sillar and Elson, 1986). CPG activity in isolated nerve cord studies in the slow walking animals implies reciprocal inhibition of oscillating networks because there are no state-dependent inputs to the isolated CNS (Delcomyn, 1980). By definition, the system cannot be state-dependent if there are no effectors to occupy a state. The crayfish model combines state-dependent inputs with an oscillating network. The robust behavior of the crayfish model suggests that both fast and slow walking animals may use a hybrid system in which the state of the legs affects the output of the oscillators.

Furthermore, behavior of real animals suggests that common principles may affect both slow and fast walkers. Crayfish may be conceptualized as faster slow-walkers compared to stick insects that are truly slow walkers. Crayfish show changes in limb function based on walking speed. Posterior legs appear to be the most active at higher velocities. At slower walking speeds, the anterior legs are more involved in the stepping pattern (Chapter 3). One species of cockroach, Periplaneta americana, runs on its back two legs only at very high speeds (Full and Tu, 1991). The animal changes gait at slower speeds and includes the anterior legs. The difference between crayfish and this cockroach may only be the range in speed. If crayfish had higher top speeds and the fluid environment permitted, they too could change posture and run on hind legs only. This, of course, is unlikely because in an aquatic environment the drag forces acting on an upright posture would probably cause the animal to flip backwards. However, the fundamental biomechanical principles are not necessarily different between these two animals as suggested by the change in anterior leg function in crayfish at different speeds. If the biomechanical principles 
are the same and fundamentally constrain gait, mechanical coordinating mechanisms between slow and fast walkers can be the same. This would further suggest that two-legged walking is governed by these same principles.

\section{Comparison to other locomotor systems and evolutionary impact}

Successful four-legged walking in the model suggests that emergent stability can also be applied to six- and four-legged rigid-bodied animals. This would include many of the walking arthropods. Further studies are required to test how effectively the hypothesis would apply to arthropods with more than eight legs, such as isopods and myriapods. Because these animals have segmented bodies, they also have additional degrees of freedom with respect to potential center of mass dynamics. They also have a wider range of potential combinations of legs to be used as bases of support. Studies on centipedes showed that these animals display a metachronal wave of leg activity (Anderson et al., 1995). This locomotor output is similar to many swimming behaviors; therefore, it may be the case that these segmented walkers have tightly coupled segmental oscillators similar to swimming animals. If this is so, then it may be evidence that walking strategies of highly segmented and fused-bodied arthropods have different evolutionary histories of their locomotor programs.

An "open" connectedness, such as the one used by the crayfish model, works especially well if the body is not segmented. Most swimming systems function in bodies that are segmented and use more control in the rhythmic muscle output for optimal propulsion. In swimming systems, each segment can be thought of as one oscillator with each half of the oscillator controlling muscle output on one side of the body. Walking systems have oscillating networks within each contralateral half of the ganglion, or one oscillator per hemiganglion (Sillar et al., 1987;

Chasserat and Clarac, 1990). Four-legged terrestrial vertebrates have commissural interneurons that influence coordination between segmental oscillators (Kato, 1990; Kjaerulff and Kiehn, 1997). As these animals evolved from segmented swimmers, they may have conserved commissural neurons across hemiganglia and developed additional oscillators within the hemiganglia that drive antagonistic muscle pairs of individual leg joints. The crayfish model produces successful, realistic walking behavior when it is reduced to a four-legged animal. 
However, it does stumble on occasion and has to reacquire the stepping rhythm. Mutual inhibition between contralateral and ipsilateral oscillators would reinforce an alternating rhythm and reduce stumbling. This is critical to four-legged animals because they do not have the luxury of multiple bases of support that additional legs would provide. However, the success of the model demonstrates properties of walking behavior that are common to invertebrates and vertebrates. The design of the vertebrate nervous system reflects these mechanical influences.

\section{Behavior as a sum of its parts}

The crayfish model reveals several aspects of locomotion as emergent properties of the collective dynamics of the nervous system, the body, legs and the environment. Any attempt to define behavior by removing contributing components, or through the components themselves, is incomplete and changes the behavior. A good example is the rhythmic excitation of the isolated nerve cord. Many, if not all, systems cannot reproduce precise output through individual components of the system or by reducing the number of integral components (Chiel and Beer, 1997). However, it is important to know the components that make up the system, or in other words, the form of the system. Without the form, it is impossible to capture the function. Conversely, function can be obtained through several configurations of unique parts. This is especially true with powerful modeling techniques such as genetic algorithms and artificial neural networks. Desired outputs can be obtained but at a potential cost to the design of the system. Form and function are so inter-related that eliminating even small pieces of one changes the output of the system. Therefore, a better approach may be to identify and include all necessary components, but to reduce the levels of complexity in individual components to determine the minimum requirements sufficient to produce real behavior. In this way, synthesis of more complex behavior has a robust template with which to start.

The culmination of the behavioral experiments led to the virtual crayfish and the hypothesis of walking stability as an emergent property in the absence of complex neural control. The model suggests a level of interrelatedness between organism and environment that has been underestimated by previous walking models. Evolved walking systems may have complex nervous systems that produce similar behavior, but these may be no more than redundant 
mechanisms that reinforce the allowable output predicated by the environment-organism dynamics. Understanding what a body can do in its environment provides an inside-out approach to the ethology of form and function.

\section{References}

Anderson, B.D., Shultz, J.W. and Jayne, B.C. (1995). Axial kinematics and muscle activity during terrestrial locomotion of the centipede Scolopendra heros. J. exp. Biol. 198, 1185-1195

Alexander, R. M. (2003). Principles of animal locomotion. Princeton University Press, Princeton, Oxford, pp 53-67

Beer, R.D. (1990). Intelligence as adaptive behavior: an experiment in computational neuroethology. Chandrasekaran, B. (ed.). Academic Press, Inc., San Diego

Blickhan, R. and Full, R.J. (1987). Locomotion energetic of the ghost crab. II. Mechanics of the center of mass during walking and running. J. exp. Biol. 130, 155-174

Borgmann, A., Scharstein, H., and Büschges, A. (2007). Intersegmental coordination: Influence of a single walking leg on the neighboring segments in the stick insect walking system. $J$. Neurophysiol. 98, 1685-1696

Cavagna, G.A., Heglund, N.C., and Taylor, C.R. (1977). Mechanical work in terrestrial locomotion: two basic mechanisms for minimizing energy expenditure. Am. J. Physiol. 233, R243-R261

Chiel, H.J. and Beer, R.D. (1997). The brain has a body: adaptive behavior emerges from interactions of nervous system, body and environment. Trends Neurosci. 20, 553-557

Chrachri and Clarac, F. (1990). Fictive locomotion in the fourth thoracic ganglion of the crayfish, Procambarus clarkii. J. Neurosci. 10(3), 707-719

Clarac, F. (1978). Locomotory programs in basal leg muscles after limb autotomy in the Crustacea. Brain Res. 145, 401-405. 
Clarac, F. and Barnes, W. J. P. (1985). Peripheral influences on the coordination of the legs during walking in decapods crustaceans. In: Coordination of motor behavior. Bush, B.M.H. and Clarac, F. (eds.). Cambridge University Press, Cambridge. pp 249-269

Clarac, F. and Chasserat, C. (1979). Experimental modifications of interlimb coordination during locomotion of a Crustacea. Neurosci. Lett. 12, 271-276

Cruse, H. (1990). What mechanisms coordinate leg movement in walking arthropods? Trends Neurosci. 13, 15-21

Cruse, H., Bartling, C., Dean, J., Kindermann, T., Schmitz, J. and Schumm, M. (2000). A simple neural network for the control of a six-legged walking system. Proc. 1993 IEEE/RSJ Int. Conf. Intell. Robot. Syst. 1, 545-552

Cruse, H., Dean, J., Dürr, V., Kindermann, T., Schmitz, J. and Schumm, M. (2002). A decentralized, biologically based network for autonomous control of (Hexapod) walking. Auton. Robots pp. 383-400

Cruse, H., Dürr, V., Schmitz, J. (2007). Insect walking is based on a decentralized architecture revealing a simple robust controller. Phil. Trans. R. Soc. A 365, 221-250

Delcomyn. F. (1980). Neural basis of rhythmic behavior in animals. Science 210, 492-498

Dudek, D. M. and R. J. Full. (2006). Passive mechanical properties of legs from running insects. J. exp Biol. 209, 1502-1515.

Full, R.J. and Koditschek, D.E. (1999). Templates and anchors: neuromechanical hypotheses of legged locomotion on land. J. exp. Biol. 202, 3325-3332

Full, R.J. and Tu, M.S. (1991). Mechanics of a rapid running insect: two-, four- and six-legged locomotion. J. exp. Biol. 156, 215-231

Goulding, M. (2009). Circuits controlling vertebrate locomotion: moving in a new direction. Nature Reviews Neuroscience. 10, 507-518 
Grillner, S., Deliagina, T., Ekeberg, O., El Manira, A., Hill, R.H., Lansner, A., Orlovsky, G.N. and Wallén, P. (1995). Neural networks that co-ordinate locomotion and body orientation in lamprey. Trends Neurosci. 18, 270-279

Grote, J. R. (1981). The effect of load on locomotion in crayfish. J. exp. Biol. 92, 277-288

Hancox, J.C. and Pitman, R.M. (1991). Plateau potentials drive axonal impulse burst in insect motoneurons. Proc. R. Soc. Lond. B 244, 33-38

Hancox, J.C. and Pitman, R.M. (1993). Plateau potentials in an insect motoneurone can be driven by synaptic stimulation. J. exp. Biol. 176, 307-310

Hill, A.A.V., Masino, M.A., and Calabrese, R.L. (2003). Intersegmental coordination of rhythmic motor patterns. J. Neurophysiol. 90, 531-538

Jamon, M. and Clarac, F. (1995). Locomotor patterns in freely walking crayfish (Procambarus clarkii). J. exp. Biol. 198, 683-700

Kato, M. (1990). Chronically isolated lumbar spinal cord generates locomotor activities in the ipsilateral hindlimb of the cat. Neurosci. Res. 9, 22-34

Kindermann, T. (2002). Behavior and adaptability of a six-legged walking system with highly distributed control. Adapt. Behav. 9, 16-41

Kjaerulff, O. and Kiehn, O. (1997). Crossed rhythmic synaptic input to motoneurons during selective activation of the contralateral spinal locomotor network. J. Neurosci. 17, 9433-9447

Kubow, T.M. and Full, R.J. (1999). The role of the mechanical system in control: a hypothesis of self-stabilization in hexapedal runners. Phil. Trans. R. Soc. Lond. B. 354, 849-862

Lim, J.L. and DeMont, M.E. (2009). Kinematics, hydrodynamics and force production of pleopods suggest jet-assisted walking in the American lobster (Homarus americanus). J. exp. Biol. 212, 2731-2745 
Namba, H. and Mulloney, B. (1999). Coordination of limb movements: three types of intersegmental interneurons in the swimmeret system, and their responses to changes in excitation. J. Neurophysiol. 81, 2437-2450

Raibert, M.H., Chepponis, M., and Brown Jr., H.B. (1986). Running on four legs as thought they were one. IEEE J. Rob. Auto. RA-2, 70-82

Ramirez, J.M. and Pearson, K.G. (1991a). Octopamine induces bursting and plateau potentials in insect neurons. Brain Res. 549, 332-337

Ramirez, J.M. and Pearson, K.G. (1991b). Octopaminergic modulation of interneurons in the flight system of the locust. J. Neurophysiol. 66, 1522-1537

Schmitt, J. and Holmes, P. (2000a). Mechanical models for insect locomotion: dynamics and stability in the horizontal plane. I. Theory. Biol. Cybern. 83, 501-515

Schmitt, J. and Holmes, P. (2000a). Mechanical models for insect locomotion: dynamics and stability in the horizontal plane. II. Application. Biol. Cybern. 83, 517-527

Sillar, K.T. and Elson, R.C. (1986). Slow active potentials in walking-leg motor neurons triggered by non-spiking proprioceptive afferents in the crayfish. J. exp. Biol. 126, 445-452

Sillar, K., Clarac, F., and Bush, B.M.H. (1987). Intersegmental coordination of central neural oscillators for rhythmic movements of the walking legs in crayfish, Pacifasticus leniusculus. J. exp. Biol. 131, 245-264

Skinner, F.K. and Mulloney, B. (1998). Intersegmental coordination of limb movements during locomotion: mathematical models predict circuits that drive swimmeret beating. J. Neurosci. 18(10), 3831-3842

Sponberg, S. and R. J. Full. (2008). Neuromechanical response of musculo-skeletal structures in cockroaches during rapid running on rough terrain. J. exp Biol. 211, 433-446.

Tschuluun, N., Hall, W.M., Mulloney, B. (2001). Limb movements during locomotion: Tests of a model of an intersegmental coordinating circuit. J. Neurosci. 21(19), 7859-7869 
Wallén, P. and Grillner, S. (1987). N-Methyl- $\mathrm{D}^{-}$-aspartate receptor induced, inherent oscillatory activity in neurons active during fictive locomotion in the lamprey. J. Neurosci. 7, 2745-2755 


\title{
Curriculum Vitae
}

\author{
Marc D. Rinehart \\ 53 Campus Dr., Life Sciences Building \\ Dept. of Biology, West Virginia University, Morgantown, WV \\ marcdrinehart@gmail.com
}

Phone: (304) 293-5201

\section{Education}

2009

Ph.D. in Biology

West Virginia University

Dissertation: Locomotion grows up: the neuromechanical control of interlimb coordinating mechanisms in crayfish

1999

B.S. in Zoology

Louisiana State University

\section{Positions Held}

$2008-2009$

Research Assistant, Department of Biology, West Virginia University

$2004-2007$

Teaching Assistant, Department of Biology, Louisiana State University

\section{Teaching Experience}

2005, 2006- 2007

2006

2004
Lab Teaching Assistant, Vertebrate Physiology (4161 LSU)

Teaching Assistant, Introduction to Biology II (1202 LSU)

Lab Teaching Assistant, Introduction to Biology I (1208 LSU) 


\section{Publications}

Vídal-Gadéa, AG, Rinehart, MD, and Belanger, JH (2008). Skeletal adaptations for forwards and sideways walking in three species of decapod crustaceans. Arthropod Struct. Dev. 37, 95108.

\section{Presentations}

Society for Neuroscience Annual Meeting, Washington, D.C., U.S.A., November, 2005

Rinehart, MD and Belanger, JH, "Does size matter? Effects of scaling on interlimb coordination during walking in crayfish."

Southeast Nerve Net, Atlanta, GA, U.S.A., February, 2006

Rinehart, MD and Belanger, JH, "Does size matter? Effects of scaling on interlimb coordination during walking in crayfish."

Society for Neuroscience Annual Meeting, Atlanta, GA, U.S.A., November, 2006

Rinehart, MD and Belanger, JH, "Comparison of the walking pattern and interlimb coordination of larval and adult crayfish."

Eighth International Congress of Neuroethology, Vancouver, BC, Canada, July 2007

Rinehart, MD and Belanger, JH, "Differences in the stepping pattern of juvenile and adult crayfish: Size or load?"

Society for Integrative and Comparative Biology Annual Meeting, Boston, MA, U.S.A., January, 2009

Rinehart, MD and Belanger, JH, "Similar motor pattern generators produce flexible walking behavior in juvenile and adult crayfish."

Society for Neuroscience Annual Meeting, Chicago, Il., U.S.A., October, 2009

Rinehart, MD and Belanger, JH. "Biologically realistic limb coordination during multilegged walking in the absence of central connections between legs." 